EGG-WM- -9043

DE91 001829

\title{
IN SITU VITRIFICATION \\ SOFTWARE REQUIREMENTS SPECIFICATION
}

Revision 1.1

\author{
William H. Grush \\ Earl S. Marwil
}

September 1990

Scientific Computing Unit

Computer Applications and Technology Transfer Group Information Resources Department

EG\&G Idaho, Inc.

Idaho Falls, Idaho 83415

Prepared for the

U. S. Department of Energy

Idaho Operations Office

Under DOE Contract No. DE-AC07-761D01570

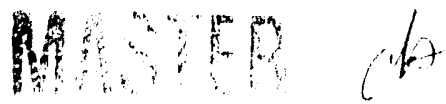

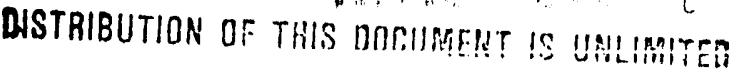


IN SITU VITRIFICATION SOFTWARE REQUIREMENTS SPECIFICATION EGG-WM-9043

Approved By:

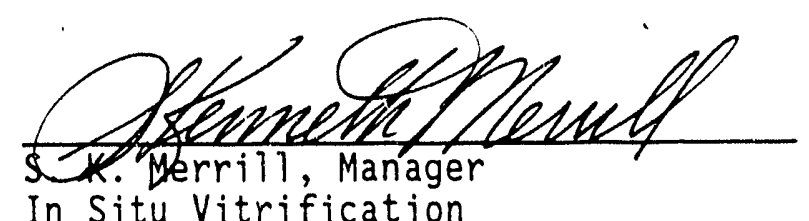

Date: $9 / 12 / 90$

In situ Vitrification

Prepared By:

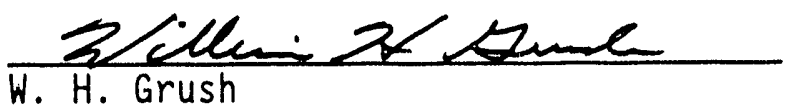

Date: $9 / 14 / 90$

Reviewed By: $\quad S D M$ athens

Date: $14 \operatorname{Sep} 90$

S. D. Matthews

Technical Reviewer 


\begin{abstract}
This report describes the Software Requirements Specification for the Electrical Resistance Heating and Thermal Energy Transport models of the In-Situ Vitrification (ISV) process. It contains the Data Flow Diagrams, Process Specifications, Data Structure Diagrams, and the Data Dictionary.
\end{abstract}




\section{CONTENTS}

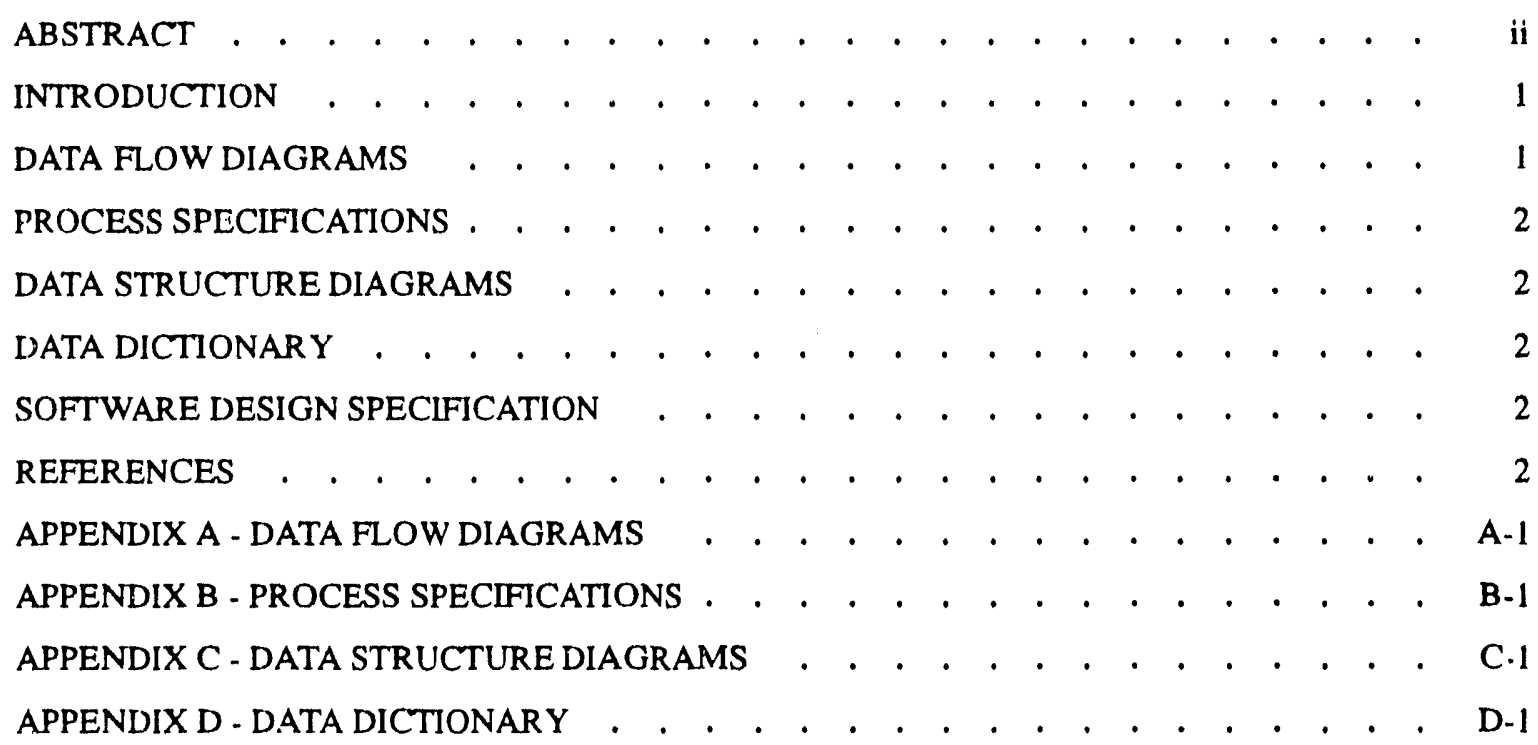




\section{IN SITU VITRIFICATION SOFTWARE REQUIREMENTS SPECIFICATION}

\section{INTRODUCTIOIN}

This software requirements specification describes the In Situ Vitrification (ISV) simulation of the coupled Electrical Resistance Heating (ERH) and Thermal Energy Transport (TET) problems. Other aspects of the ISV modeling effort are to be simulated with stand alone models. These other models include the process chemistry, gas release, melt flow, vapor transport, and combustion in the hood. Experimental results and analysis with stand alone simulation models may indicate that coupling of these other simulations to the ERH and TET problems is required. This software requirements specification allows as much flexibility as possible for that contingency. For a full discussion of all of the physical models considered for the ISV, the reader shoul1 refer to the ISV Modeling Requirements [1] and the ISV Implementation Plan [2].

A software requirements specification is a model on paper of the intended software system. Details of the data structures and functional processes are given and analyzed for consistency and completeness. This is a critical part of any software development to ensure that all of the requirements are incorporated in the software. The following sections briefly describe the data flow diagrams, the data structure diagrams, and the data dictionary. All of these diagrams are maintained by a software tool kit called Software through Pictures (StP) licensed to EG\&G by Interactive Development Environments (IDE) [3]. This allows a great deal of flexibility in developing the software requirements to try various scenarios and keep th: ideas best suited to the project. Additionally, the software requirements are easily maintainable. In particular, for the ISV project, the requirements for software modeling are likely to change frequently, based on the ongoing experimental program and other analysis associated with this project.

\section{DATA FLOW DIAGRAMS}

The data flow diagrams for the ISV simulation of the ERH and TET problems are included as Appendix A. The diagrams are organized starting at the "top" level or context level diagram. Level " 0 " is decomposed to show the controlling executive process through which the user can choose input data preparation, simulation, or viewing the simulation output.

The input processor manages all of the user interface in building the problem data file and the geometry file used by the simulation processor. A binary format for the files was chosen to facilitate the Quality Assurance (QA) of the problem definition associated with each simulation run. The user is allowed to change or create problem definitions only through interaction with the input processor.

The simulation processor is given only the name of the problem, which identifies the input data file and is used to construct all file names used or crealed by the simulation processor. The simulation models may be run independently for software verification and validation. As coupled nodels the ERH problem is solved at the beginning of each TET time step. The ERH problem is a static or steady state problem in this context, given the electrode potentials, the depth of the electrodes, and the prescribed boundary conditions. To account for the cycie of positive and negative voltage applied to each of four electrodes, two static problems are solved and the results averaged to produce the element heat generation rates. The TET problem is then solved for a given tisi, step, using the heat generation rate within each element computed by the ERH model. The results of both models are stored at specified intervals for subsequent postprocessing.

The output processor allows the user to view the simulation results in either a tabular printed format for selected problem variables or in a more convenient graphic format. 


\section{PROCESS SPECIFICATIONS}

At the lowest level in a data flow description is the process specifications. These are written in pseudo-code and generally correspond to a module which can be implemented as code. Each process specification (pspec) perforins a single function or task. The pspecs are included as Appendix B.

\section{DATA STRUCTURE DIAGRAMS}

The data structure diagrams for the ISV simulation of the ERH and TET problems are included as Appendix C. The data structure diagrams show the hierarchy of data structure defined for the software.

\section{DATA DICTIONARY}

The data dictionary for the ISV simulation of the ERH and TET problems is included as Appendix D. The dat a dictionary describes each data element as having a data type, constraints, a value, and other attributes as necessary. At a minimum, to be a data element, a type must be specified. Types may be integer, real, ingical, or any other standard data type or a data type specified in the software requirements. For

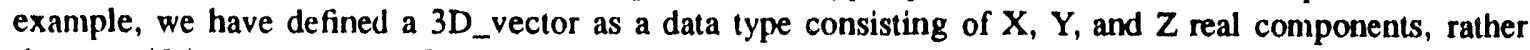
than specifying components for every vector as a unique data structure.

\section{SOFTWARE DESIGN SPECIFICATION}

The next stage of the software development is to derive a software architecture from the software requirements. This document represents a snapshot of the requirements at this time. The software requirements may change as a result of changing modeling requirements or questions raised during design specification. Therefore, the final authority on the software requirements specification is the on-line diagrams, process specifications, and data dictionary as maintained in StP.

\section{REFERENCES}

[1] R.J. MacKinnon, D.C. Mecham, et al., Modeling Requirements for In Situ Vitrification, EG\&G Idaho, Inc. Draft Repor, December 1989.

[2] R.J. MacKinnon, et al., In Situ Vitrification Model Development and Implementation Plan, EG\&G Idaho, Inc. Draft Report, March 1990.

[3] Software Through Pictures, Release 4.2, Interactive Development Environments (IDE), San Francisco, CA, March 1990.

[4] R.S. Pressman, Software Engineering, A Practitioner's Approach, McGraw-Hill, San Francisco, CA, 1987.

[5] E. Yourdon, Modern Structured Analysis, Yourdon Press, Englewood Cliffs, NJ, 1989. 


\title{
APPENDIX A - DATA FLOW DIAGRAMS
}

\author{
Diagram: top \\ I)iagram: 0 \\ Dingram: 2 \\ Diagram: 2.1 \\ Diagram: 3 \\ Diagram: 3.3 \\ Diagram: 3.3 .2 \\ Diagram: 3.3 .3 \\ Dingram: 3.3 .4 \\ Dingram: 3.3 .5 \\ Diagram: 3.3 .7 \\ Diagram: 3.4 \\ Diagran: 3.4 .2 \\ Diagram: 3.4 .2 .5 \\ Diagram: 3.4 .3 \\ Diagram: 3.4 .4 \\ Diagram: 3.4 .5 \\ Diagram: 3.4 .7 \\ Diagram: 4
}


Project: In Situ Vitrification

Diagram: Iop

Name: context

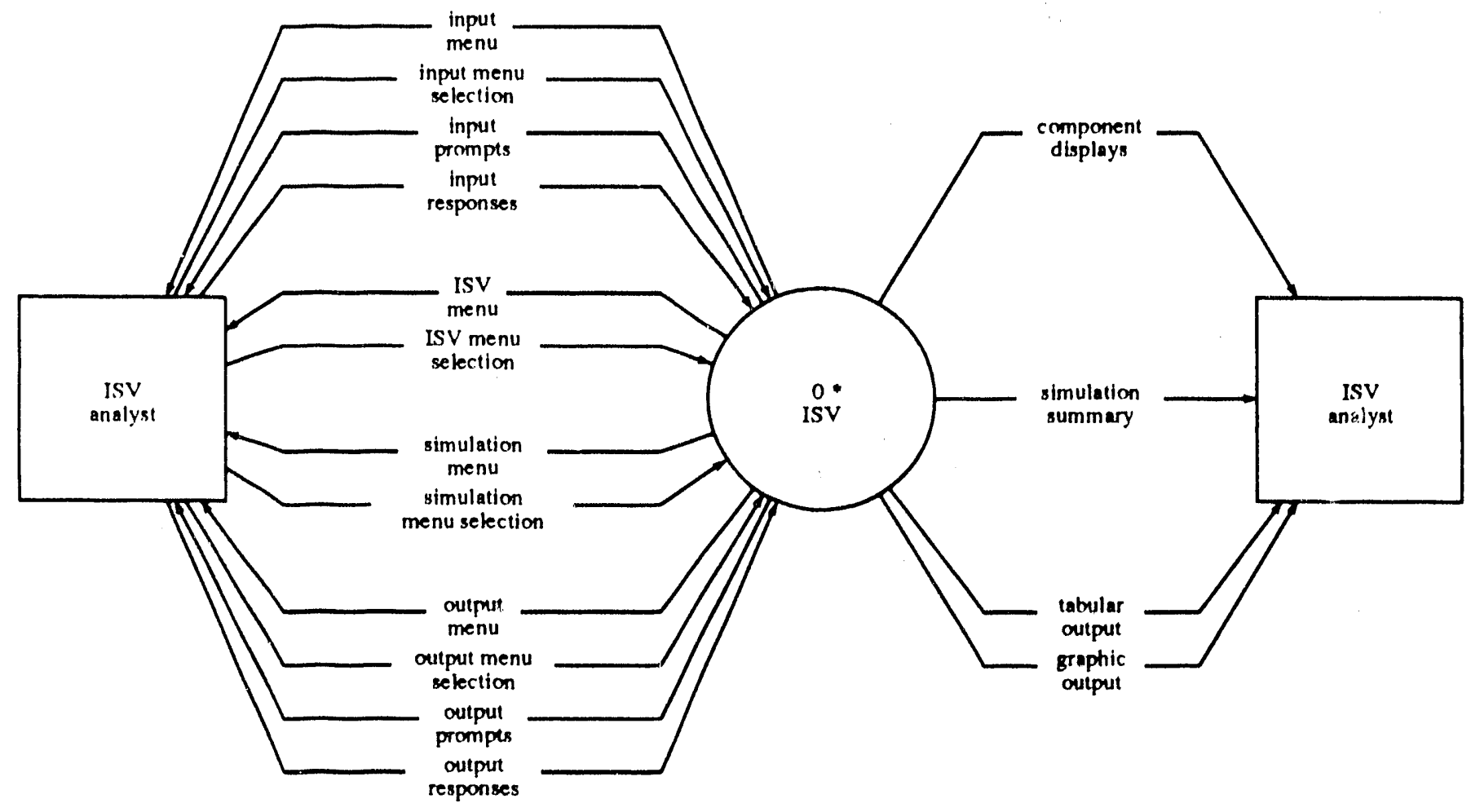


Project: In Situ Vitrification

Diagram: 0

Name: ISV

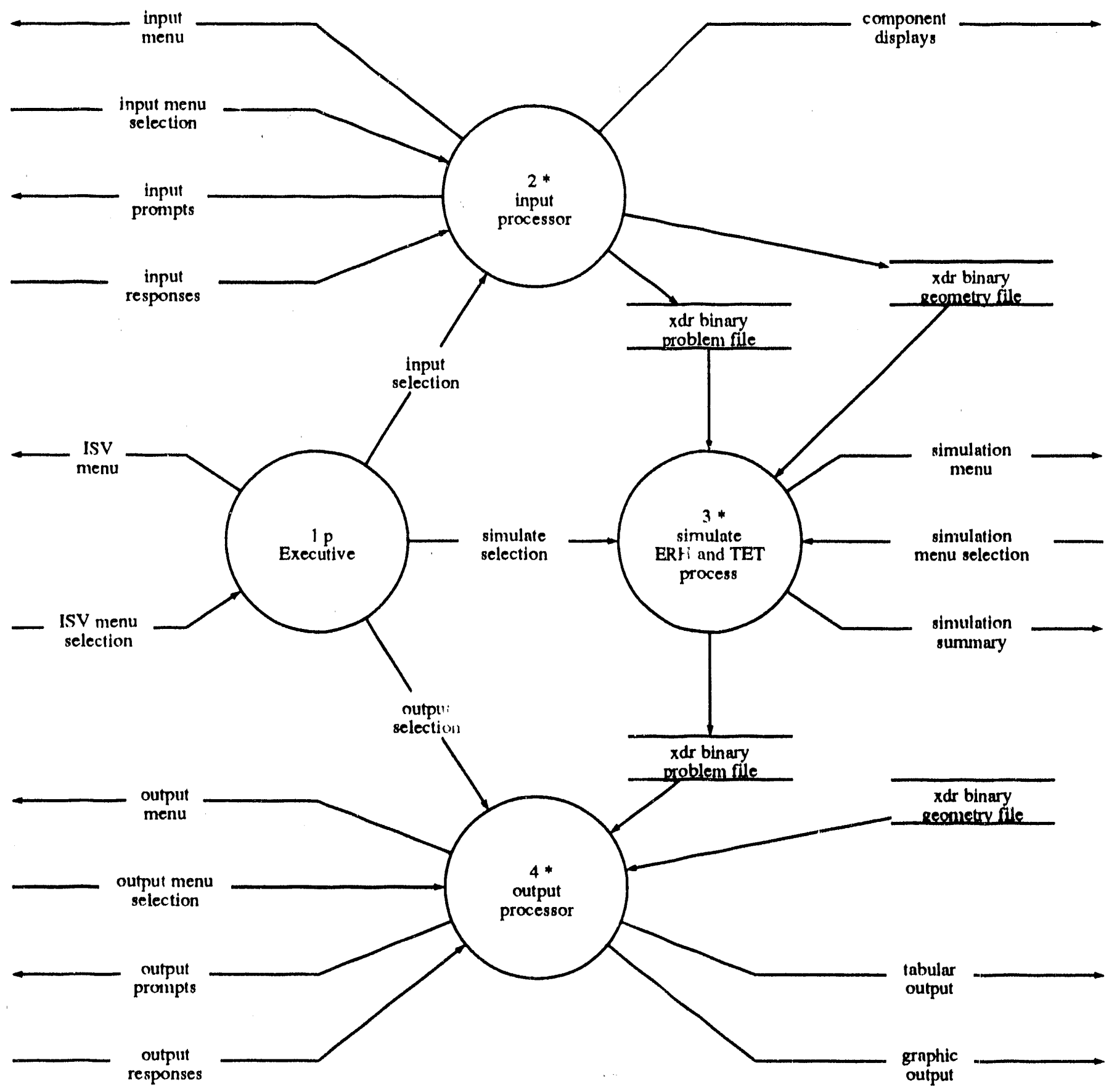


Project: In Situ Vitrification

Dingram: 2

Name: input_processor

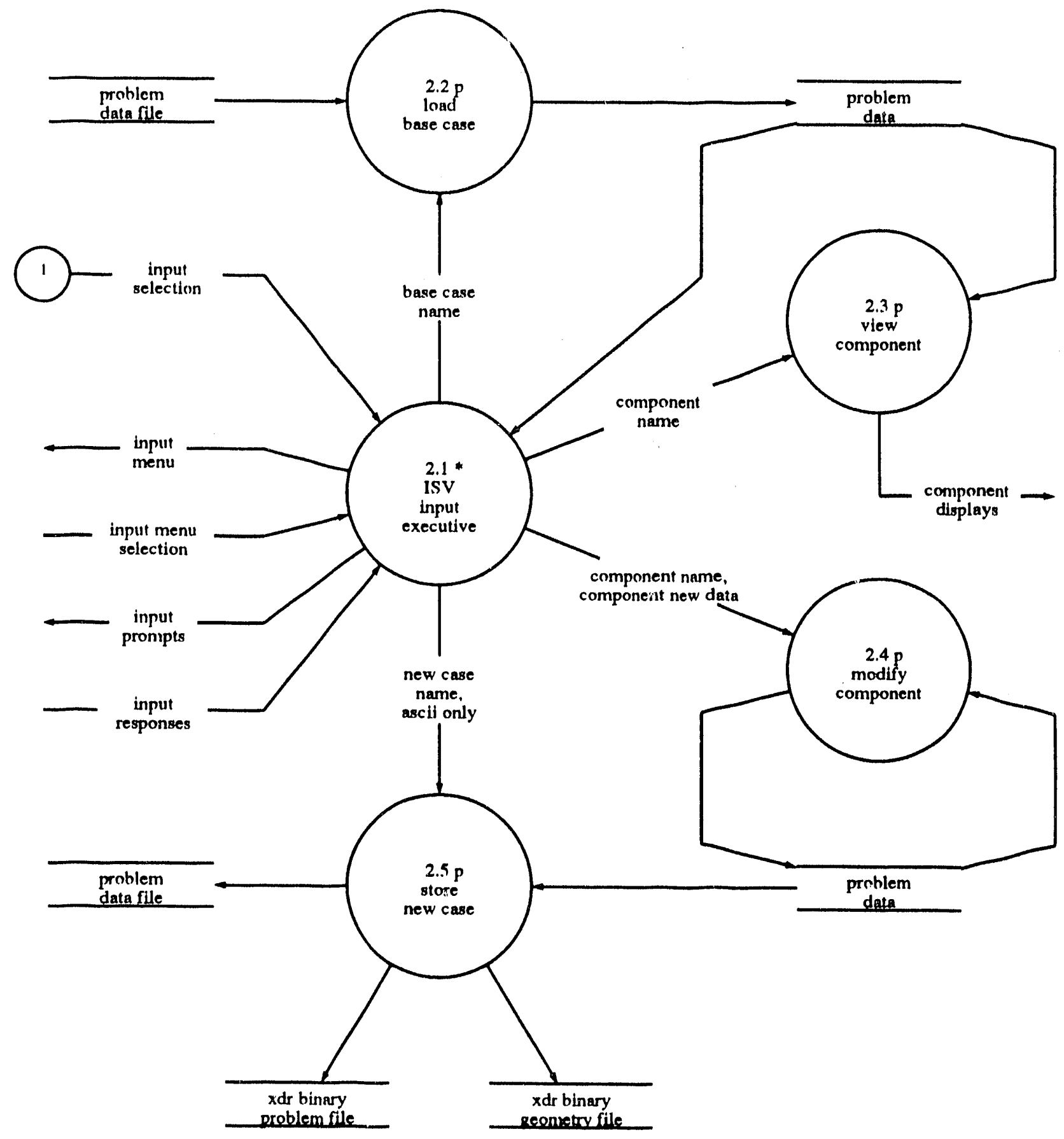

A -4 
Project: In Situ Vitrification

Diagram: 2.1

Name: ISV_input_executive

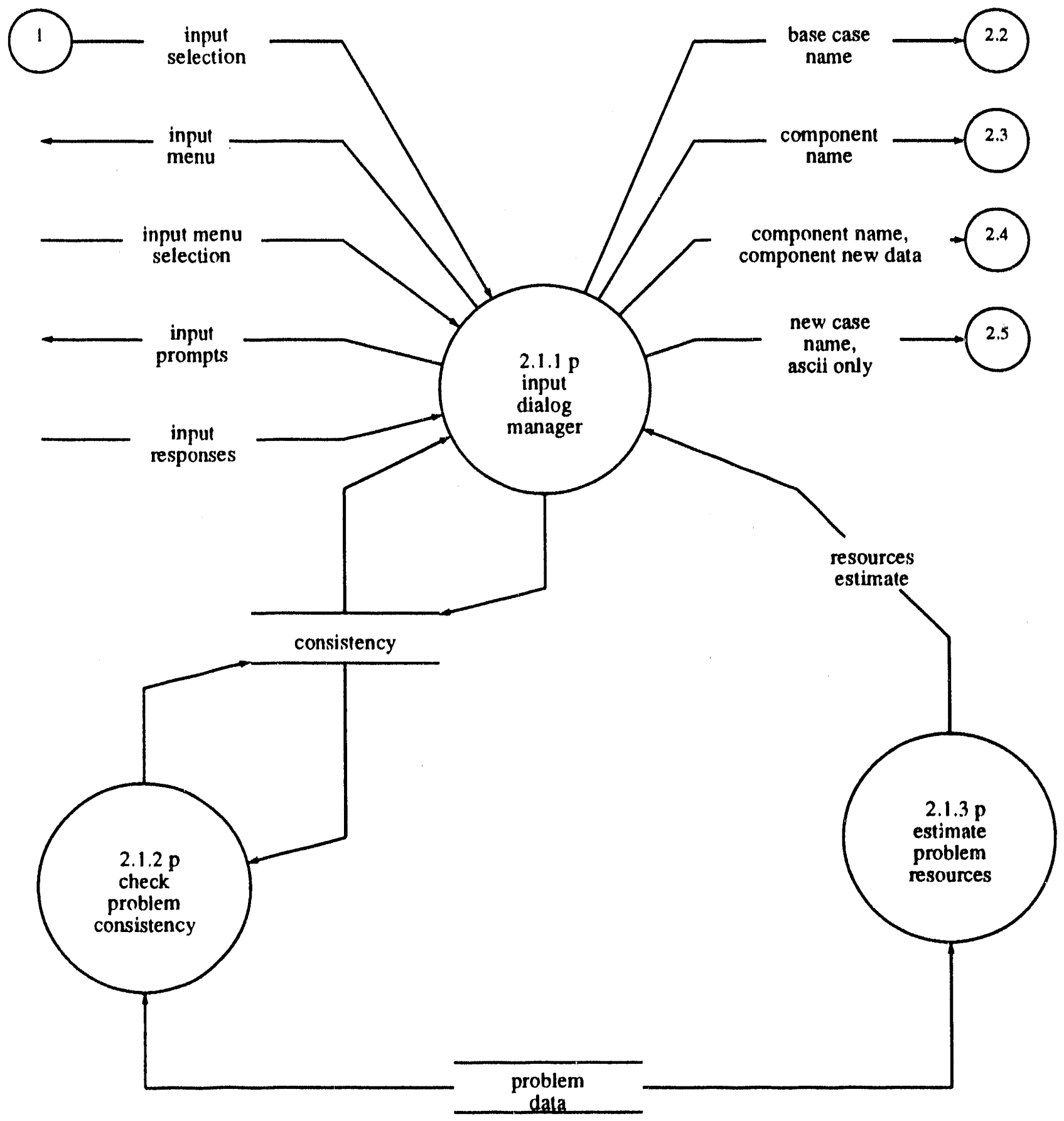

A - 5 
Project: In Situ Vitrification

Diagram: 3

Name: simulate_ERH_and_TET_process

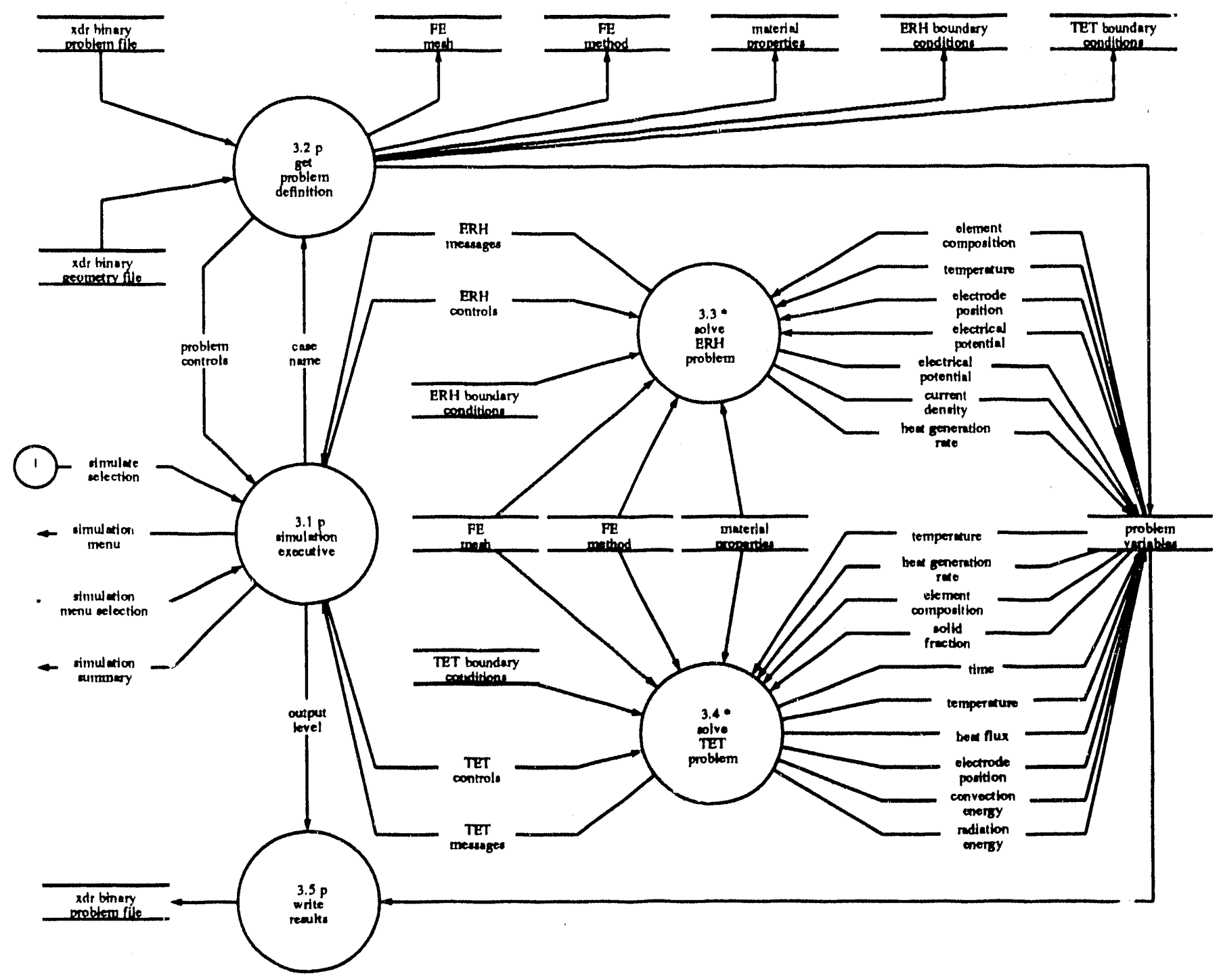


Project: In Situ Vitrification

Diagram: 3.3

Name: solve_ERH_problem

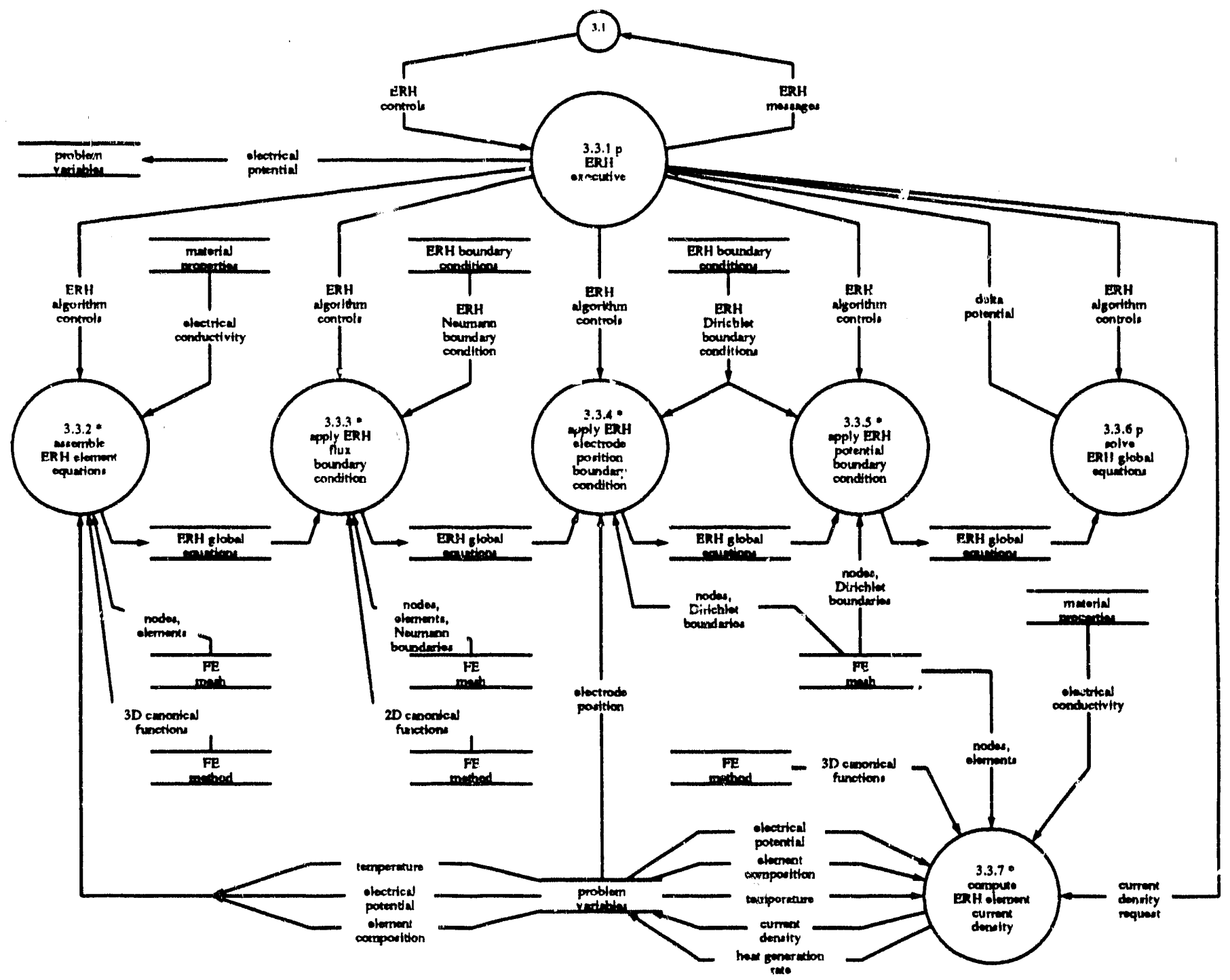


Project: In Situ Vitrification

Diagram: $\mathbf{3 . 3 . 2}$

Name: assemble_ERH_element_equations

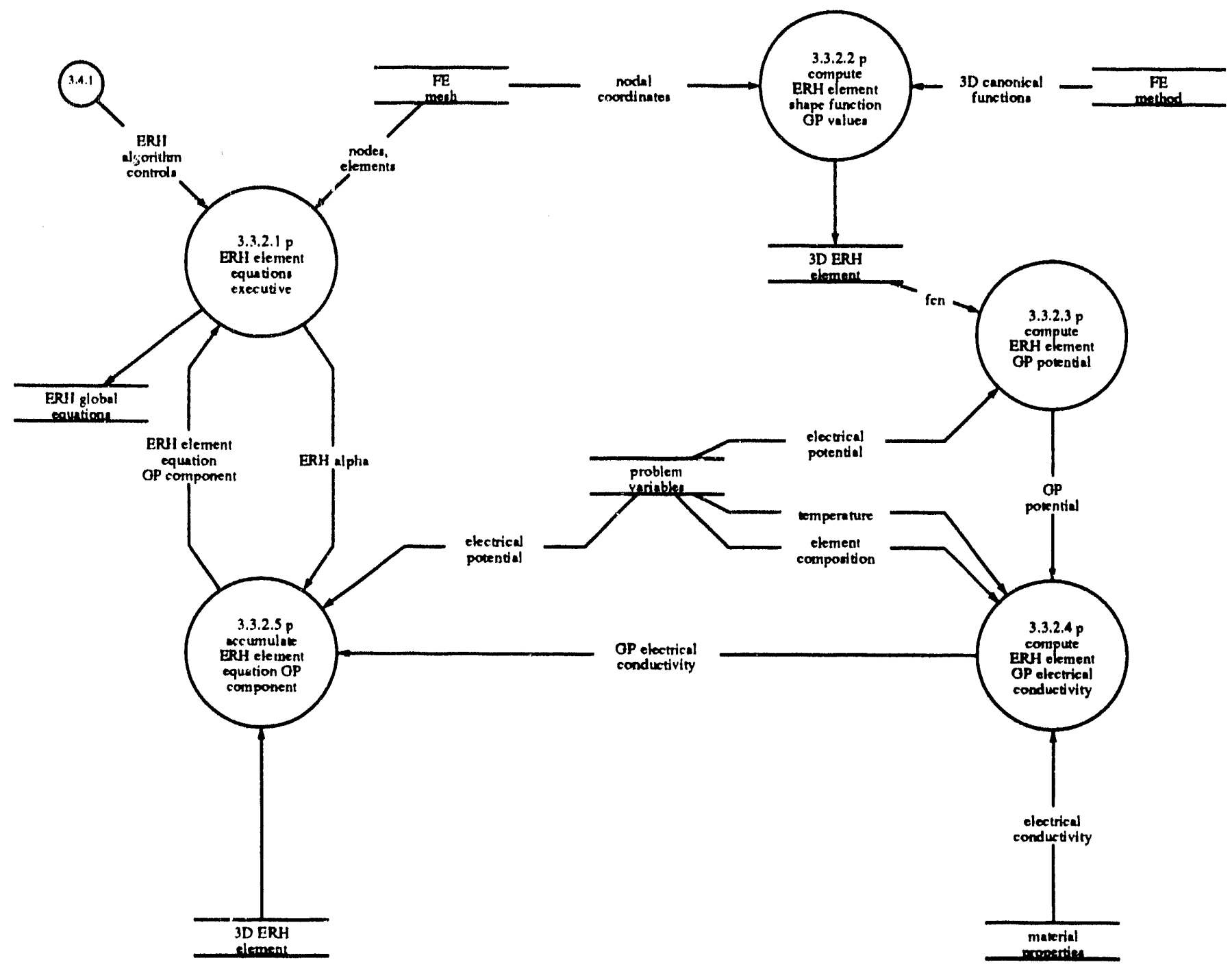


Project: In Situ Vitrification

Diagram: 3.3.3

Name: apply_FRH_flux_boundary_condition

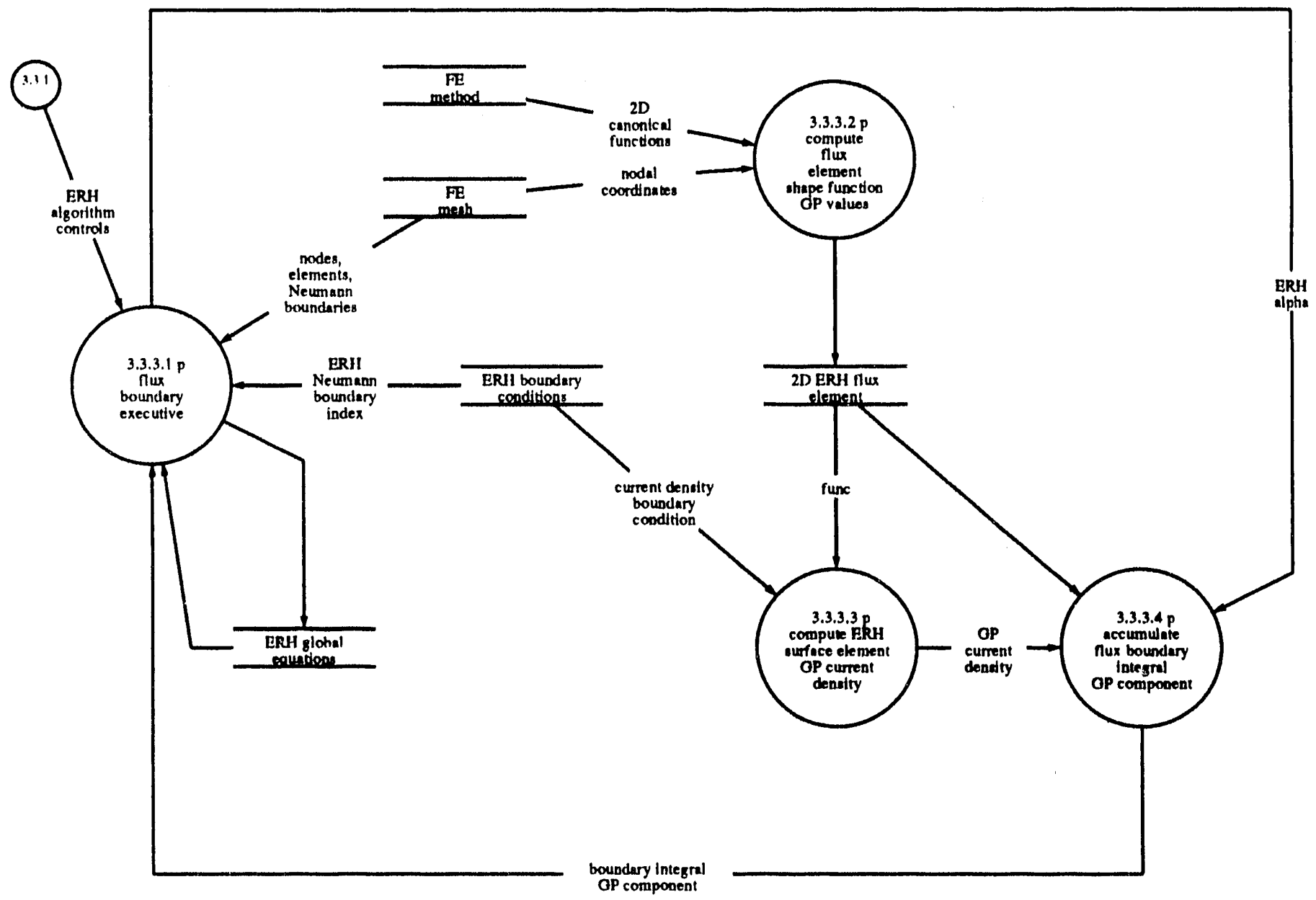

A - 9 
Project: In Situ Vitrification

Diagram: $\mathbf{3 . 3 . 4}$

Name: apply_ERH_electrode_position_boundary_condition

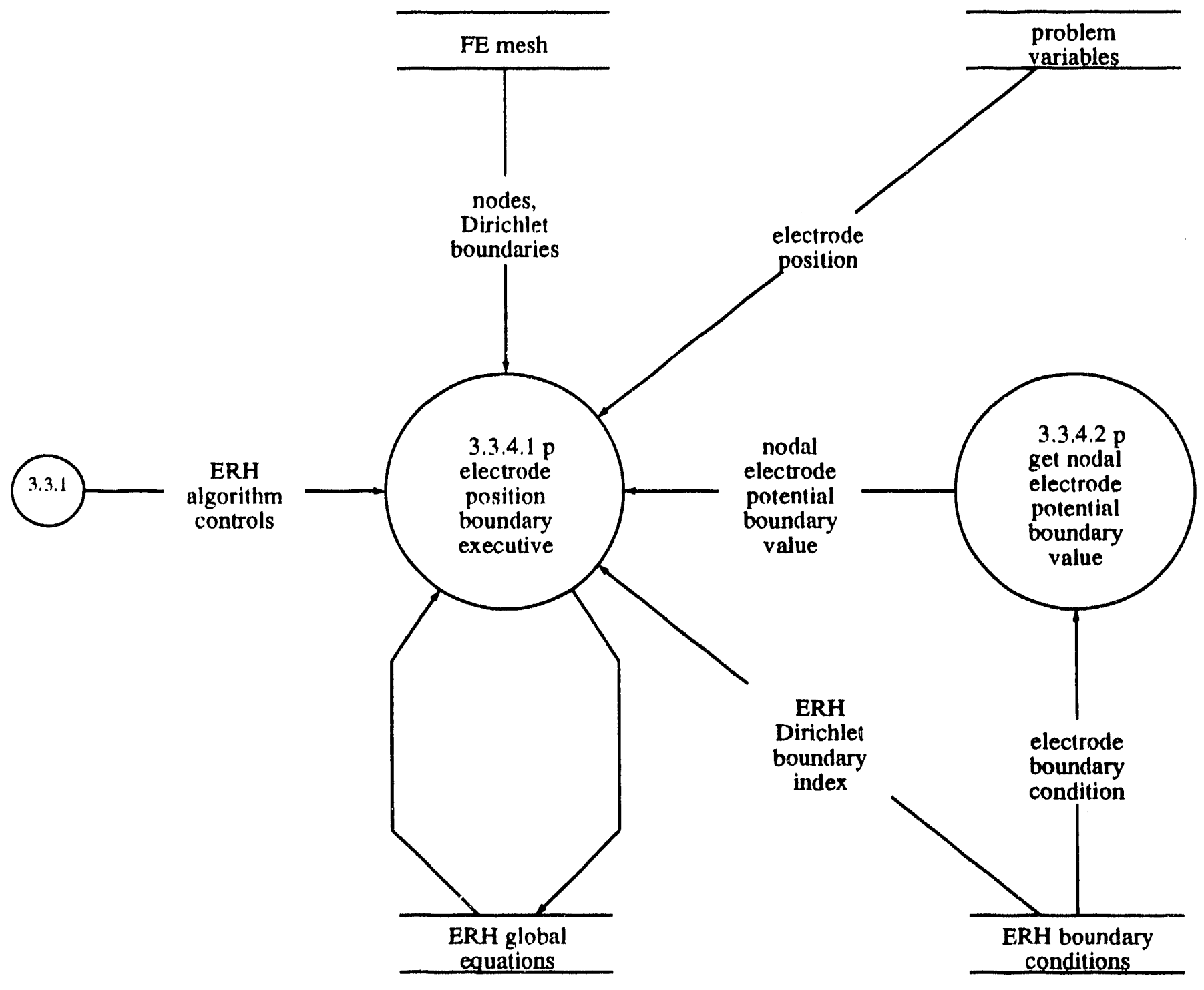


Project: In Situ Vitrification

Diagram: 3.3 .5

Name: apply_ERH_potential_boundary_condition

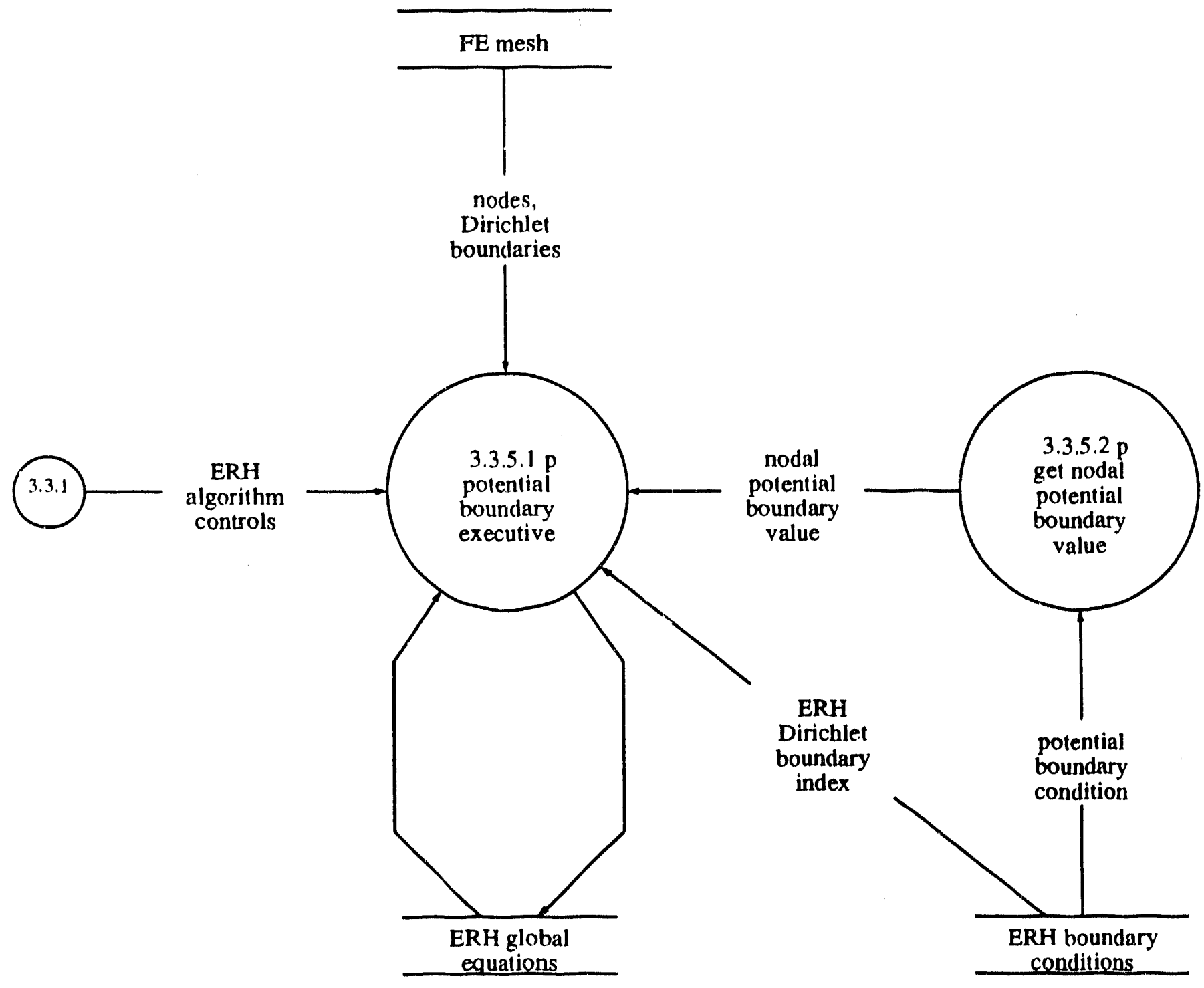


Project: In Situ Vitrification

Diagram: 3.3.7

Name: compute_ERH_element_current_density

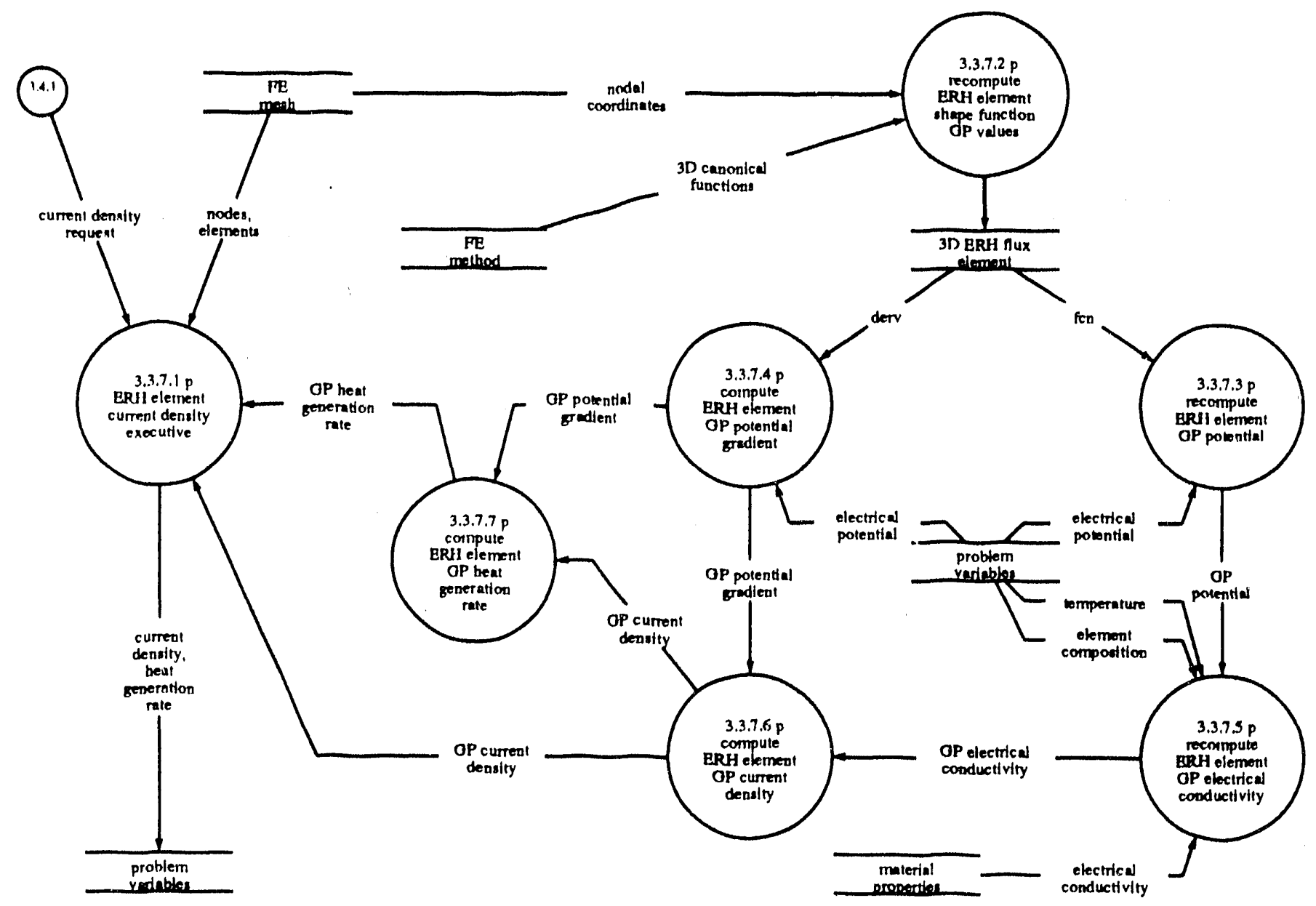


Project: In Situ Vitrification

Dingram: 3.4

Name: solve_TET_problem

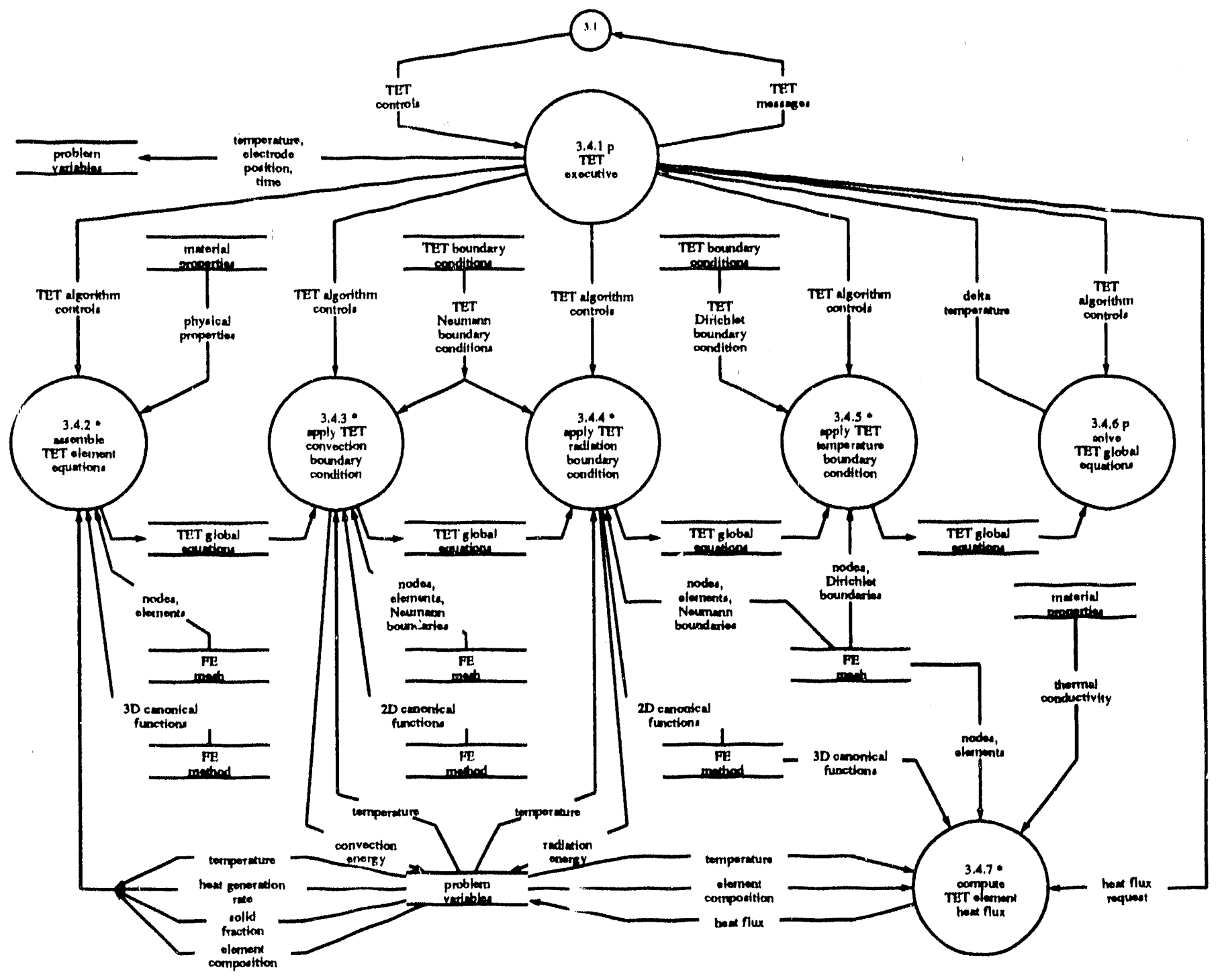


Project: In Situ Vitrification

Diagram: 3.4 .2

Name: assemble_TET_element_equations

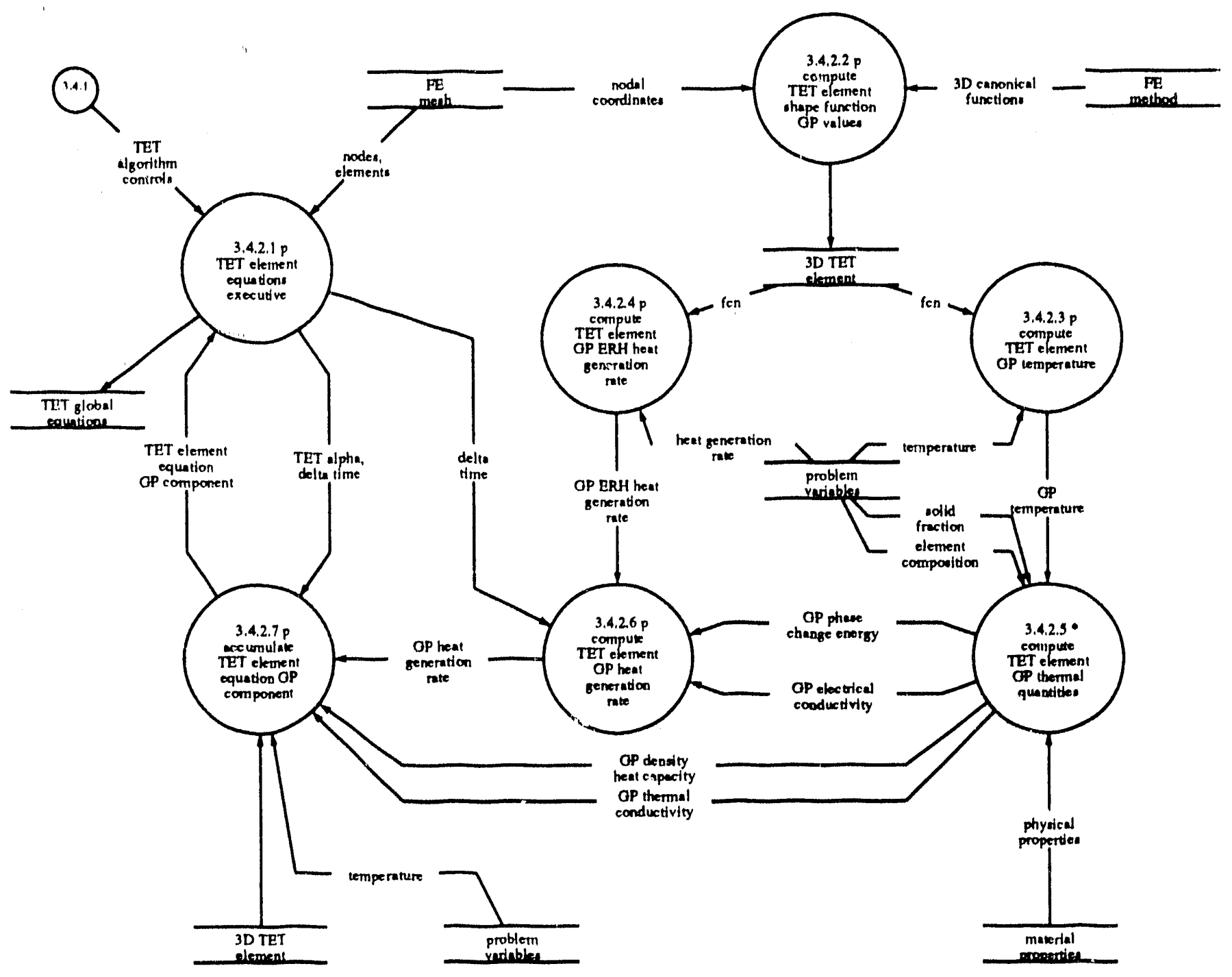


Project: In Situ Vitrification

Diagram: 3.4.2.5

Name: compute_TET_element_GP_thermal_quantities

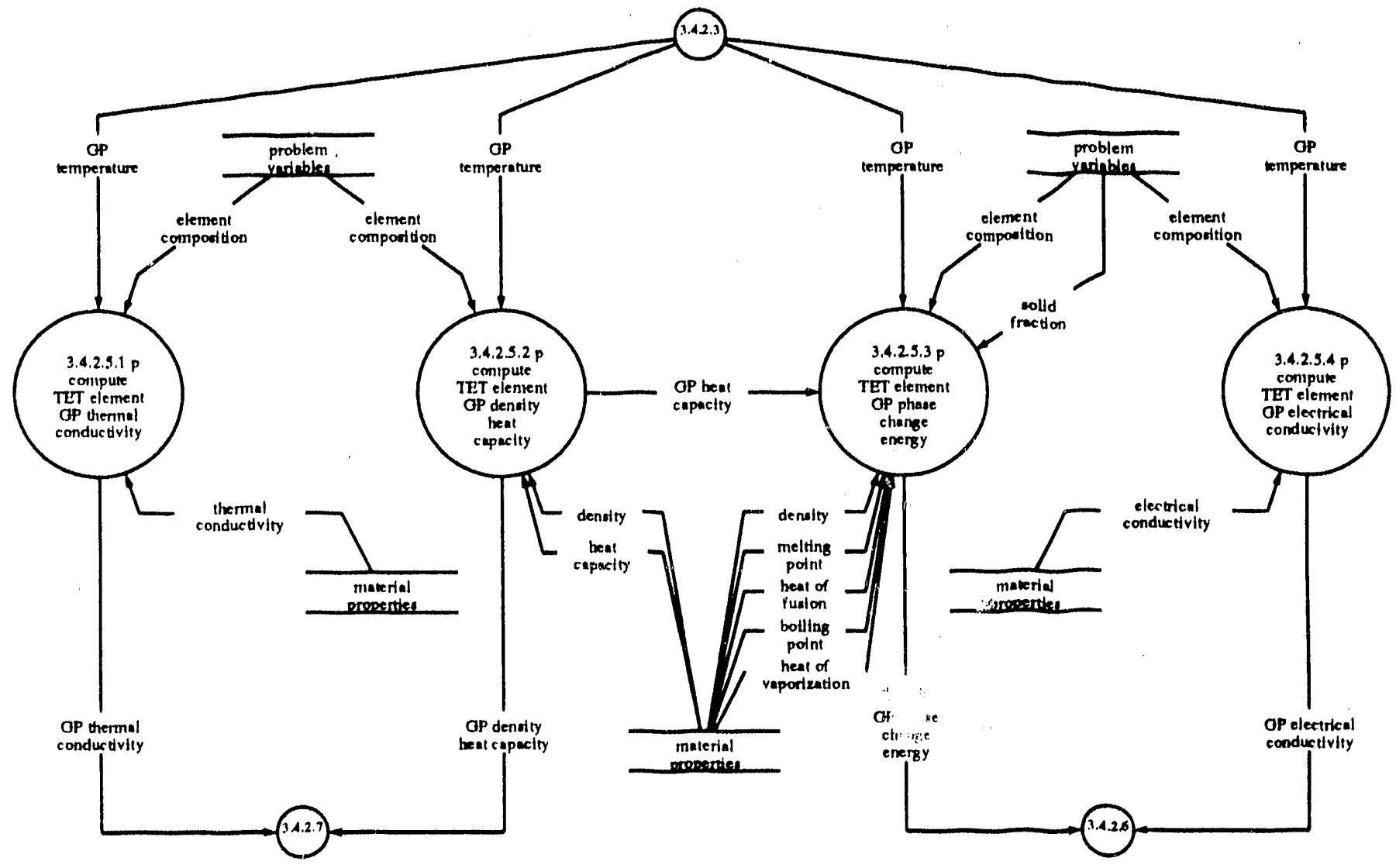


Project: In Situ Vitrification

Diagram: 3.4 .3

Name: apply_TET_convection_boundary_condition

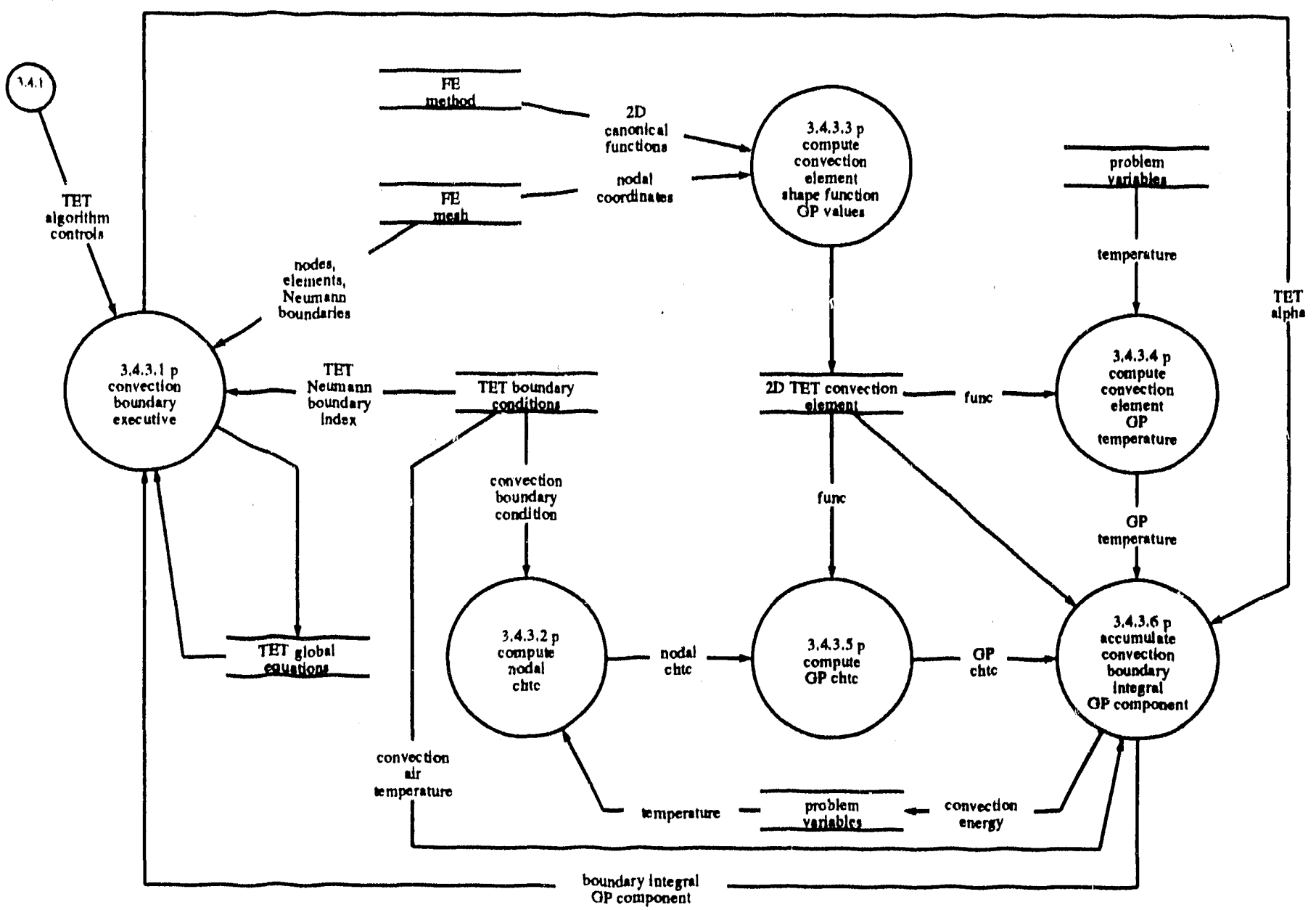


Project: In Situ Vitrification

Diagram: 3.4 .4

Name: apply_TET_radiation_boundary_condition

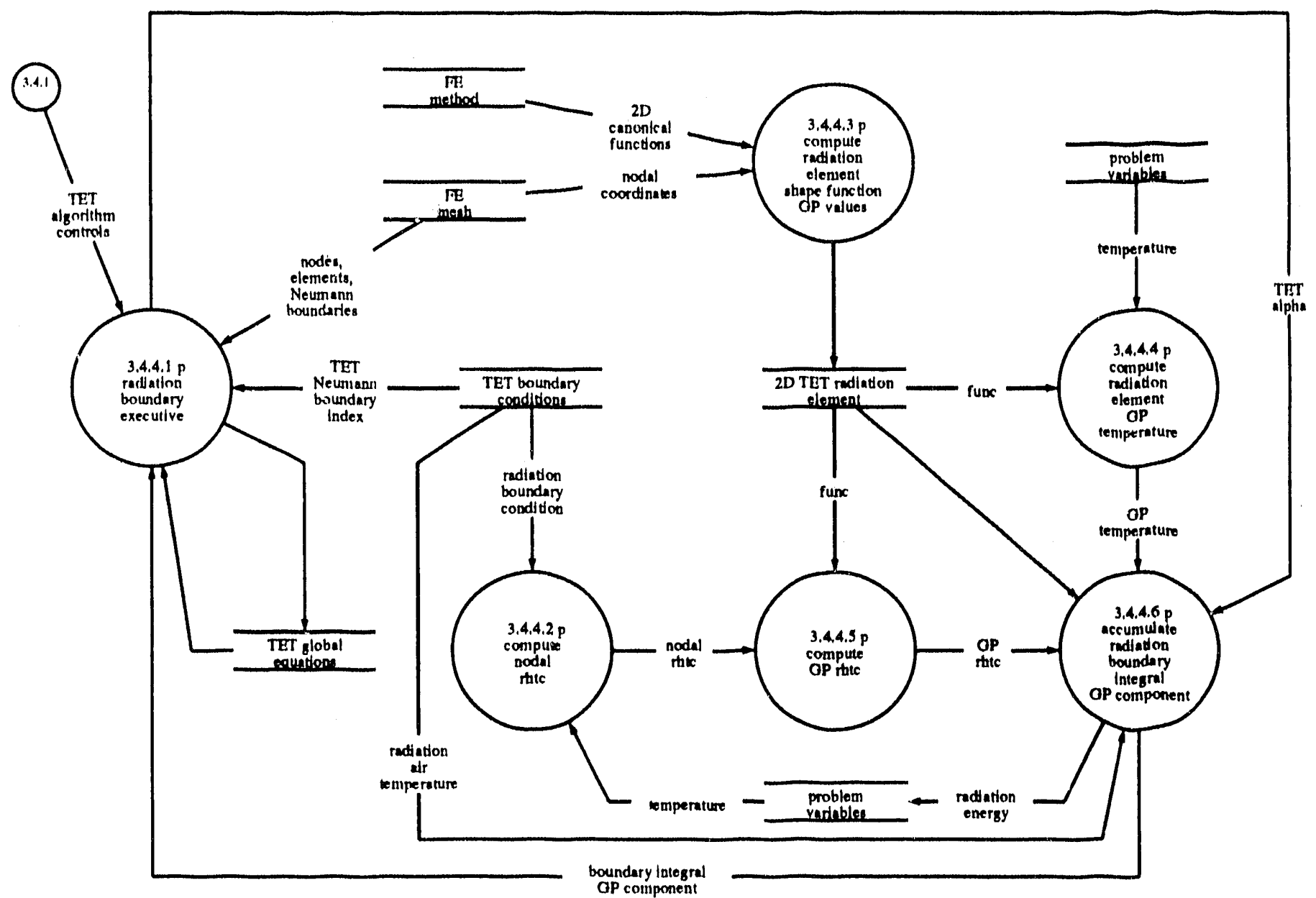

A. 17 
Project: In Situ Vitrification

Diagram: $\mathbf{3 . 4 . 5}$

Name: apply_LET_temperature_boundary_condition

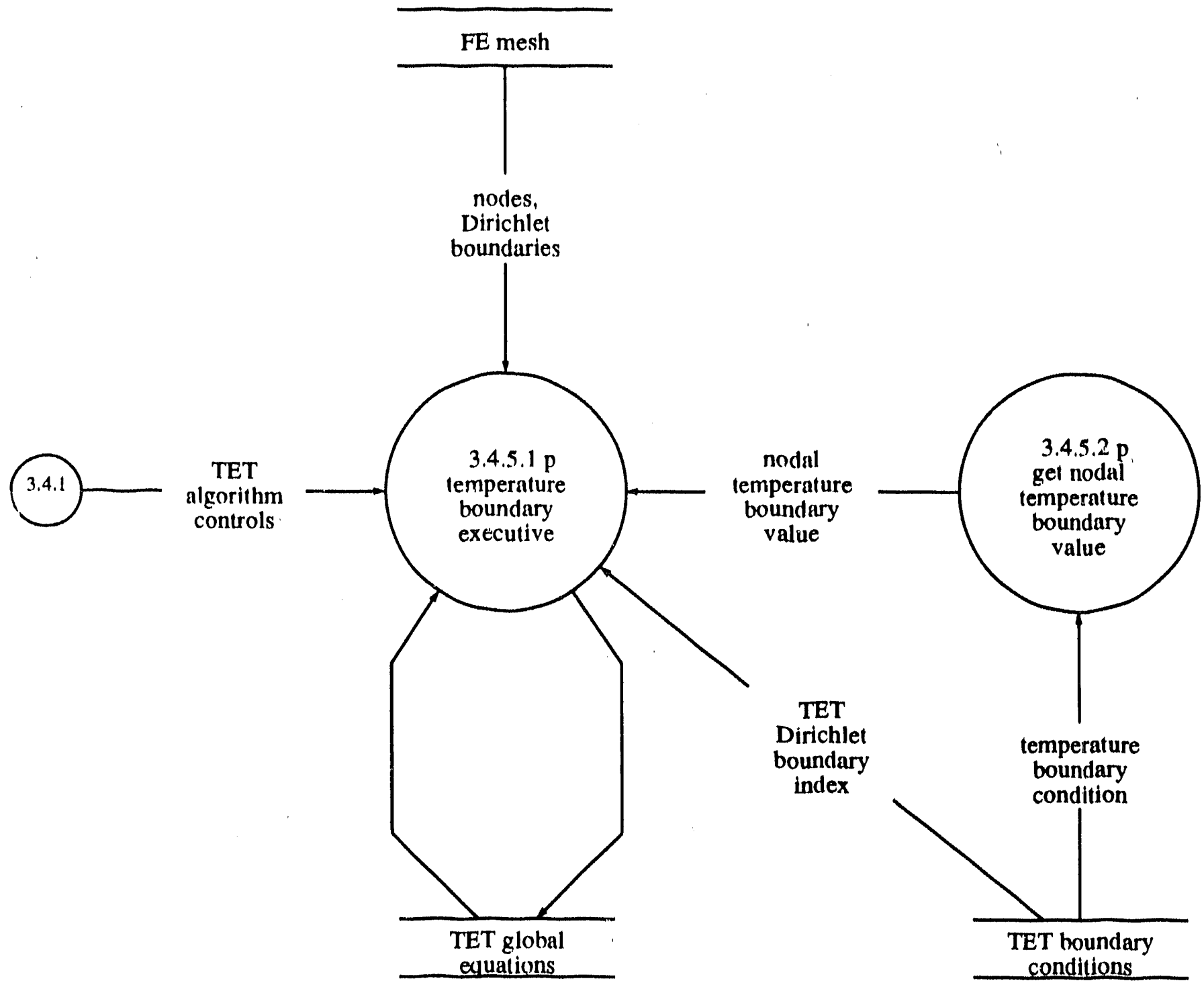

A -18 
Project: In Situ Vitrification

Dingram: 3.4.7

Name: compute_TET_element_heat_flux

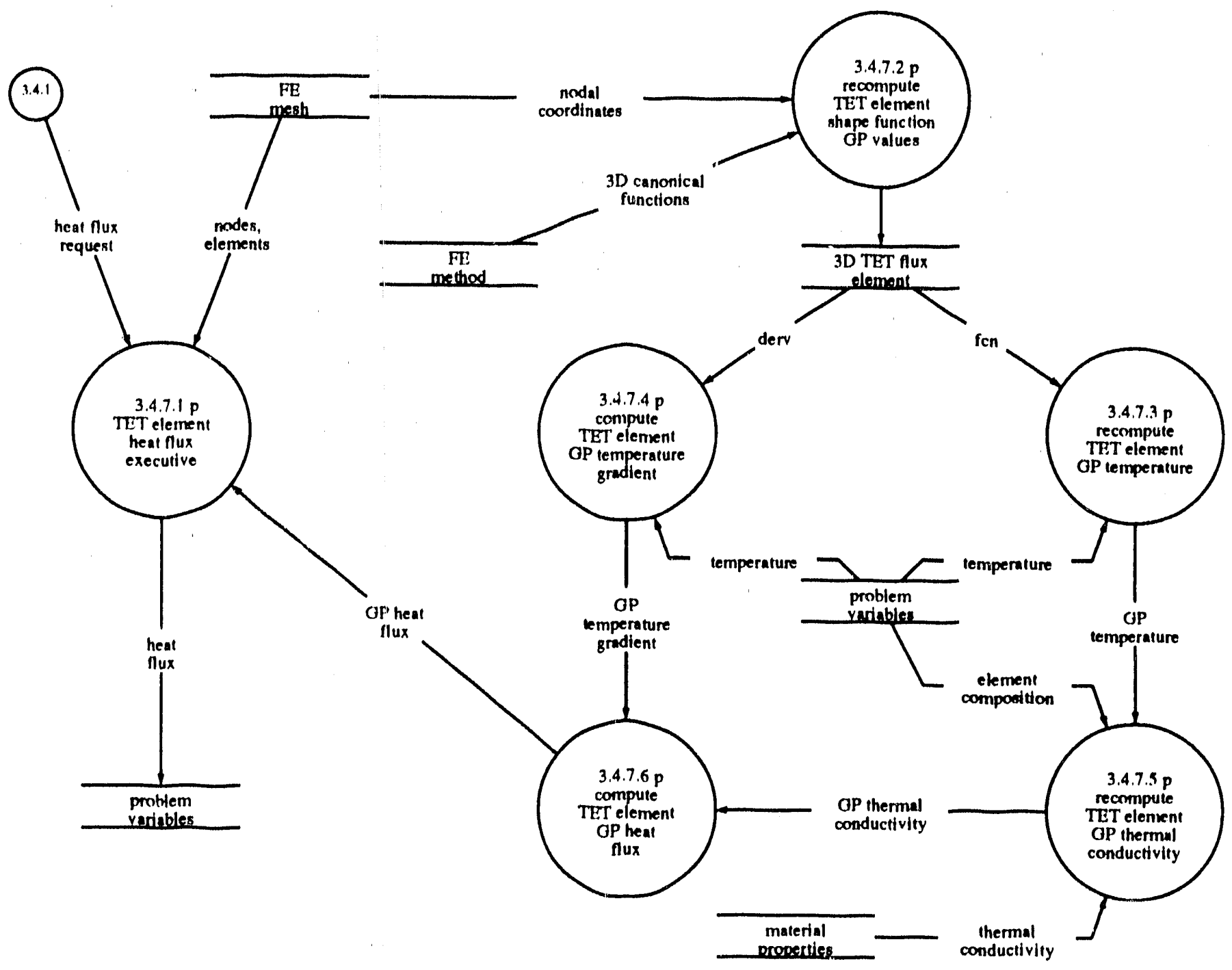

A -19 
Project: In Situ Vitrification

Diagram: 4

Name: output_processor

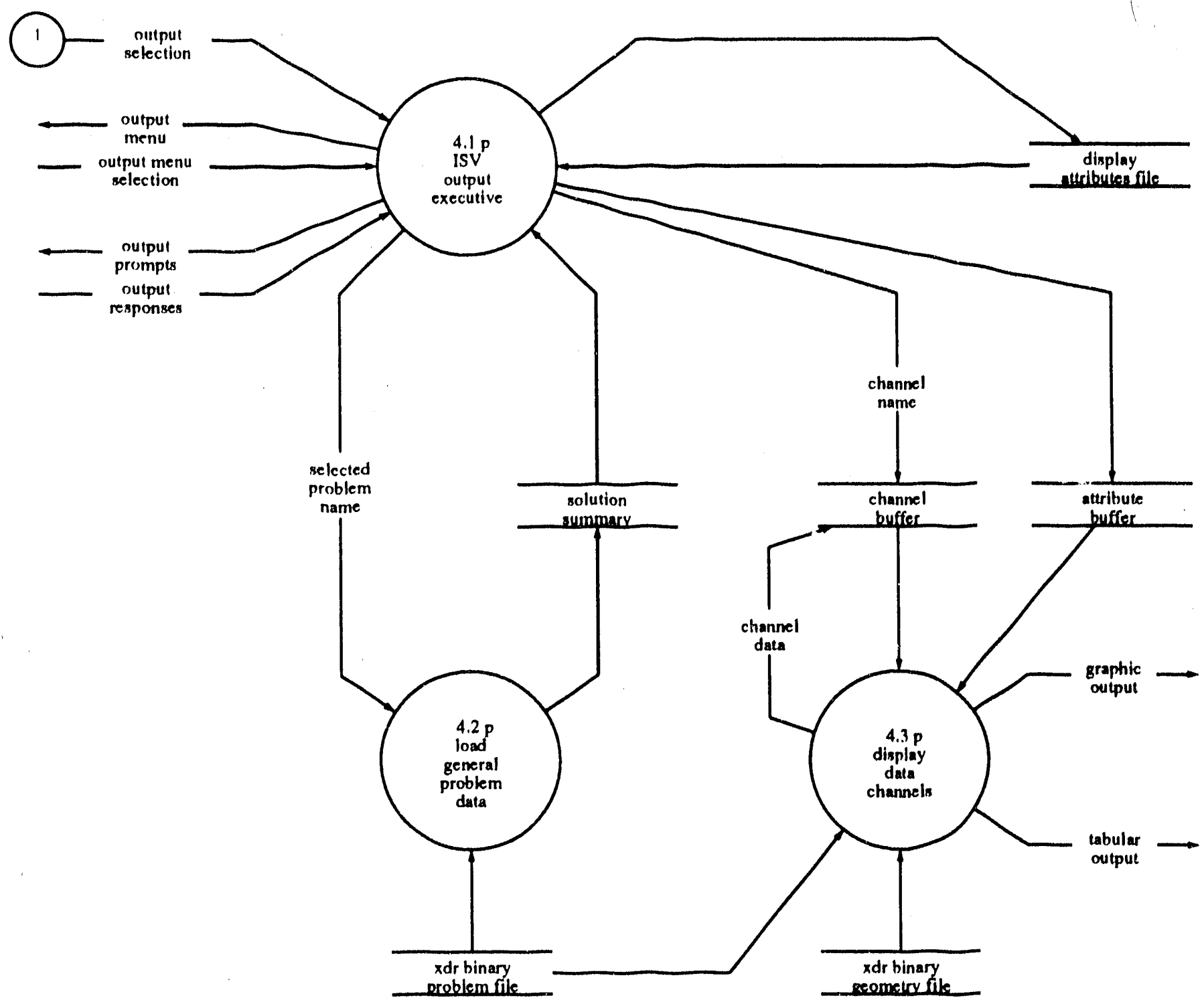




\section{APPENDIX B - PROCESS SPECIFICATIONS}

Process 1: Executive

Process 2.2: load_base_case

Process 2.3: view_component

Process 2.4: modify_component

Process 2.5: store_new_case

Process 2.1.1: input_dialog_manager

Process 2.1.2: check_problem_consistency

Process 2.1.3: estimate_problem_resources

Process 3.1: simulation_executive

J"rocess 3.2: get_problem_de finition

Process 3.5: write_results

Process 3.3.1: ERH_exc cutive

Process 3.3.6: solve_ERH_global_equaunns

Process 3.3.2.1: ERH_element_equations_executive

Process 3.3.2.2: compute_ERH_element_shape_function_GP_values

Process 3.3.2.3: compute_ERH_element_GP_potential

Process 3.3.2.4: compute_ERH_element_GP_electrical_conductivity

Process 3.3.2.5: accumulate_ERH_element_equation_GP_component Process 3.3.3.1: flux_boundary_executive

Process 3.3.3.2: compute_flux_element_shape_function_GP_values

Process 3.3.3.3: conpute_ERH_surface_element_GP_current_density

Process 3.3.3.4: accumulate_flux_boundary_integral_GP_component

Process 3.3.4.1: electrode_position_boundary_executive

Process $3.3 .4 .2:$ get_nodal_electrode_potential_boundary_value

Process 3.3.5.1: potential_boundary_executive

Process 3.3.5.2: get_nodal_potential_boundary_value

Process 3.3.7.1: ERH_element_current_density_executive

Process 3.3.7.2: recompute_ERH_element_shape_function_GP_values

Process 3.3.7.3: recompute_ERH_element_GP_potential

Process 3.3.7.4: compute_ERH_element_GP_potential_gradient

Process 3.3.7.5: recompute_ERH_element_GP_electrical_conductivity

Process 3.3.7.6: compute_ERH_element_GP_current_density

Process 3.3.7.7: compute_ERH_element_GP_heat_generation_rate

Process 3.4.1: TET_executive

Process 3.4.6: solve_TET_global_equations

Process 3.4.2.1: TET_element_equations_es rcutive

Process 3.4.2.2: compute_TET_clement_shape_function_GP_values

Process 3.4.2.3: compute_TET_element_GP_temperature

Process 3.4.2.4: compute_TET_element_GP_ERH_heat_generation_rate

Process 3.4.2.6: compure_TET_element_GP_heat_generation_rate

Prncess 3.4.2.7: accumulate_TET_element_equation_GP_component

Process 3.4.2.5.1: compute_TET_element_GP_thermal_conductivity

Process 3.4.2.5.2: compute_TET_element_GP_density_heat_capacity

Process 3.4.2.5.3: compute_TET_element_GP_phase_change_energy

Process 3.4.2.5.4: compute_TET_element_GP_electrical_conducivity

Process 3.4.3.1: convection_boundary_executive

Process 3.4.3.2: compute_nodal_clitc

Process 3.4.3.3: conpute_convection_element_shape_function_GP_values 
Process 3.4.3.4: compute_convection_element_GP_temperature

Process 3.4.3.5: compute_GP_chtc

Process 3.4.3.6: accumulate_convection_boundary_integral_GP_component

Process 3.4.4.1: radiation_boundary_executive

Process 3.4.4.2: compute_nodal_rhtc

Process 3.4.4.3: compute_radiation_element_shape_function_GP_values

Process 3.4.4.4: compute_radiation_element_GP_temperature

Process 3.4.4.5: compute_GP_ritc

Process 3.4.4.6: accumulate_radiation_boundary_integral_GP_component

Process 3.4.5.1: temperature_boundary_executive

Process 3.4.5.2: get_nodal_temperature_boundary_value

Process 3.4.7.1: TET_element_heat_flux_executive

Process 3.4.7.2: recompute_TET_element_shape_function_GP_values

Process 3.4.7.3: recompute_TET_element_GP_temperature

Process 3.4.7.4: compute_TET_element_GP_temperature_gradient

Process 3.4.7.5: recompute_TET_element_GP_thermal_conductivity

Process 3.4.7.6: compute_TET_element_GP_heat_flux

Process 4.1: 1SV_output_executive

Process 4.2: Ind_'general_problem_data

Process 4.3: display_data_channels 


\section{Executive}

Process 1: Executive

Pspec generated 4 September 1990 at 14:57:19

This process has 5 data flows:

input data flows

ISV_menu_selection

output data flows

ISV_menu

input_selection

simulate_selection

outpul_selection

description

Get command line options

Repeal 1

Display ISV_menu

Receive ISV_menu_selection

Switch (ISV_menu_selection) |

case input:

Initiate imput_process break;

case simulation:

Initiate simulation

break;

case output:

Initiate output_process

break;

case quit:

Exit;

| Until ()

I end switch

end pspec

B - 3 
Process 2.2

load_base_case

Process 2.2: load_base_case

Pspec generated 4 September 1990 at 14:58:03

This process has 3 data flows:

input data flows

base_case_name

problem_data_file

output data flows

problem_data

description

Foreach component of problem_data

Set default values

End

Foreach componet of base_case_data

End Read problem component_data

end pspec 
Process 2.3

view_component

Process 2.3: view_component

Pspec generated 4 September 1990 at 14:58:06

This process has 3 data flows:

input data flows

component_name

problem_data

output data flows

component_displays

description

Define display_description using component_name

Creale display using component_data and display_description

end pspec 


\section{Process 2.4}

modify_component

Process 2.4: modify_component

Pspec genernted 4 September 1990 at 14:58:09

This process has 3 data flows:

input dara flows

component_name

component_new_data

problem_data

output dita flows

problem_data

description

Load component_data into save buffer

While (new_component_data) ।

Replace component_data with new_component_data

I end while

If ( undo ) then

Replace component_data with save buffer

else

Store modified_component_data

endif

end pspec 
Process 2.5

store_new_case

Process 2.5: store_new_case

Pspec generated 4 September 1990 at 14:58:12

This process has 6 data flows:

input data flows

tiew_case_name

ascii_only

problem_data

output data flows

xdr_binary_problem_file

xdr_binary_genmetry_file

problem_data_file

description

Store problem_data to new_case_data_file

If ( not ascii_cnly) then

Store geometry description to xdr_binary_geometry_file

endif

Store problem description to xdr_binary_problen__tile

end pspec 
Prncess 2.1.1

input_dialog_manager

Process 2.1.1: input_dialog_manager

Pspec generated 4 September 1990 at 14:58:51

This process has 13 data flows:

input data flows

input_menu_selection

input_responses

input_selection

resources_estimate

consistency

output dnta finws

input_menu

input_prompts

base_case_name

component_name

component_name

component_new_data

new_case_name

ascii_only

consistency

description

Display input_menu

Display resources estimate

Get input_menu_selection

Switch (input_menu_selection) 1

case load:

Gel list of problem names

Display list of problem names

Prompt for selection

Get base_case_name

Set all consistency flags

Invoke load process

break;

case view:

Display list of component names

Prompt for selection

Get component_name

Invoke view process

break;

case modify:

Prompt for new component data

Get new_component data

Set component consistency flag

Invoke modify process

break;

case store:

Check consistency flags

if ( consistent) then 


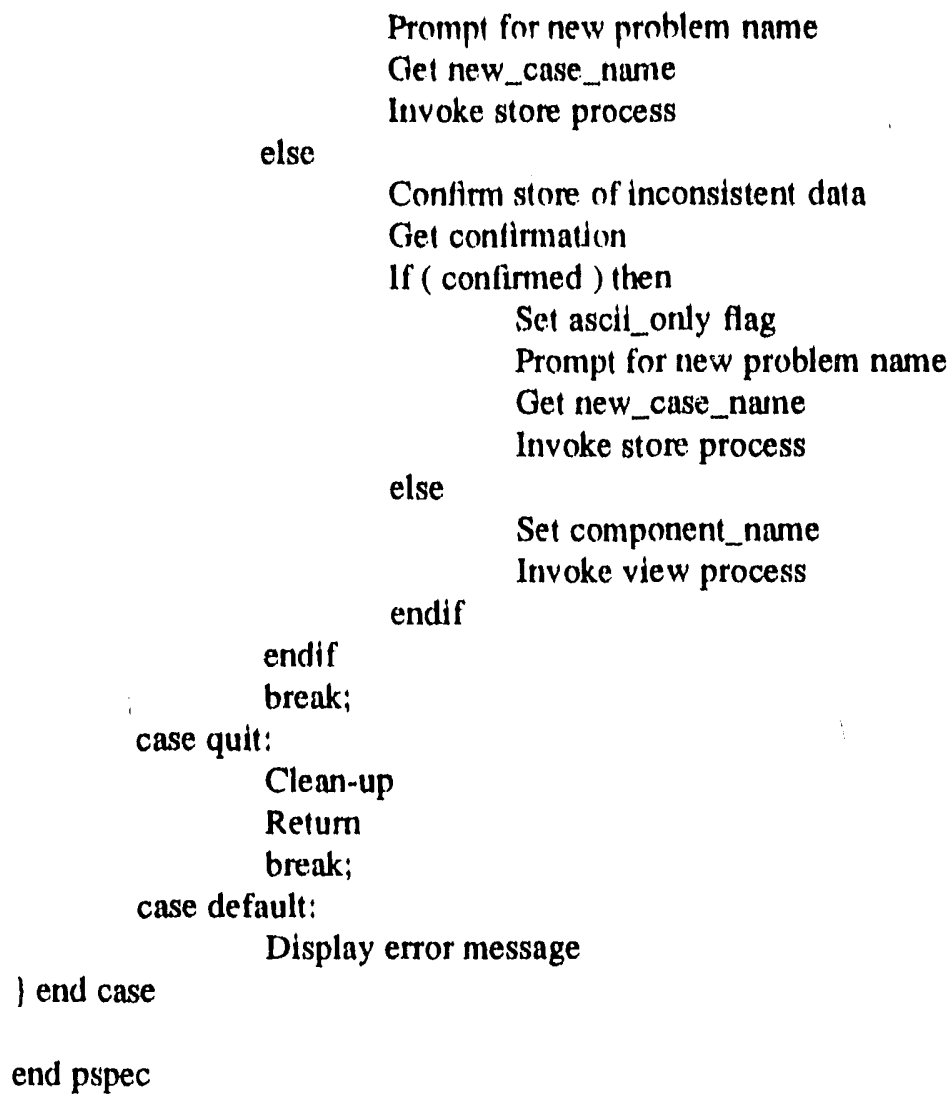




\section{Process 2.1.2}

check_problem_consistency

Process 2.1.2: check_problem_consistency

Pispec generated 4 September $199($ at $14: 58: 57$

This process hus 2 data flows:

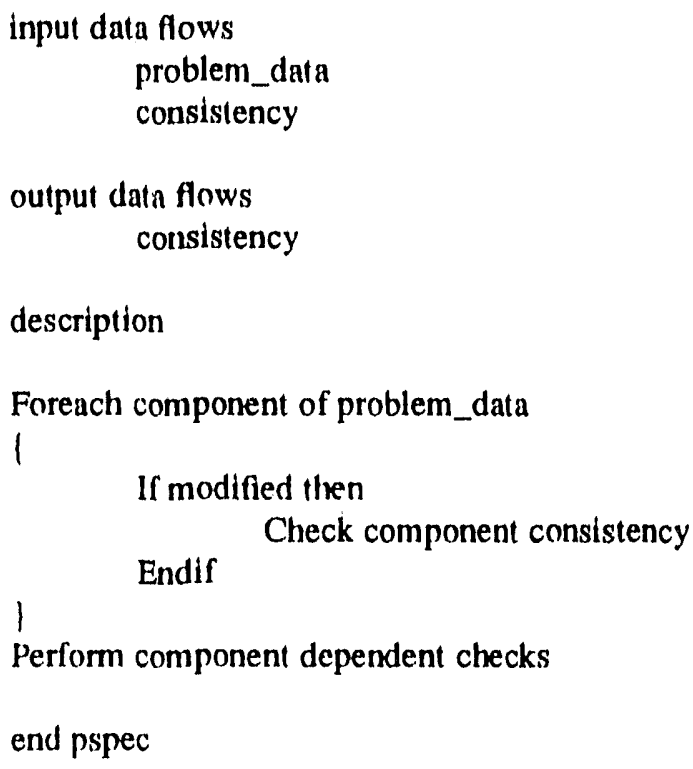


Process 2.1.3

estimate_problem_resources

Process 2.1.3: estimate_problem_resources

Pspec generated 4 September 1990 at 14:59:01

This process has 2 dala flows:

input data flows

problem_data

output data flows

resources_estimate

description

Estimate cpu

Estimate memory

Estimate disk

Display resources estimate

end pspec 
Process 3.1

simulation_executive

Process 3.1: simulation_executive

Pspec genernted 4 September 1990 at 14:59:51

This process has 11 data flows:

inpul data flows

simulate_selection

sinulation_menu_selection

problem_controls

ERH_messages

TET_messages

output data flows

simulation_summary

ERH_controls

TET_controls

output_level

case_name

simulation_menu

description

Get list of case names

Display case names

Get case_nume

Invoke get_problem_definition

Set ERH initial values

Set TET initial values to zero

Write initial values

Repeat 1

Solve ERH problem (solve number_of_electrodes $/ 2$ problems and average)

Solve TET problem

Write results using output_frequency

| Until ( completed or terminated)

Produce simulation summary

end pspec 
Process 3.2

get_problem_de finition

Process 3.2: get_problem_de finition

Pspec generated 4 September 1990 at 14:59:41

This process has 10 data flows:

input data flows

case_name

xdr_binary_geometry_file

xdr_binary_problem_file

output data flows

problem_controls

problem_variables

ERH_boundary_conditions

TET_boundary_conditions

FE_mesh

FE_method

material_properties

description

Get case geometry from xdr_binary_geometry_file

Load FE_mesh, FE_method, material_composition, and boundary_conditions

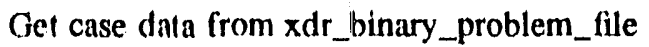

Load problem_variables

Return problem_controls

end pspec 
Process 3.5

write_results

Process 3.5: write_results

Pspec generated 4 September 1990 at 14:59:47

This process has 3 data flows:

input data flows

output_level

probleın_variables

output data flows

xdr_binary_problem_file

description

Receive output_level

Receive problem variables

Switch output_level (defined_levels) I

case 0 :

write level 0 values

break;

case 1:

write level $I$ values (plus level 0 )

break;

case 2:

write level 2 values (plus level 1 )

break;

1

end pspec 
Process 3.3.1

\section{ERH_executive}

Process 3.3.1: ERH_executive

Pspec generated 4 September 1990 at 15:00:53

This process has 10 data flows:

input data flows

ERH_controls

delta_potential

output data flows

current_density_request

ERH_messages

ERH_algorithm_controls

electrical_potential

ERH_algorithm_controls

ERH_algorithm_controls

ERH_algorithin_controls

ERH_Ilgorithm_controls

description

Repeat I

form_ERH_global_equations I

assemble ERH element equations

apply flux boundary conditon

apply electrode boundary conditon

apply potential boundary conditon

1

solve_ERH_global_equations

If (ERH_iteration_limit reached) then terminate ERH problem

endif

| Until (ERH_relative_tolerance )

update electrical_potential values

compute_current_density

end pspec 
Process 3.3.6

solve_ERH_global_equations

Process 3.3.6: solve_ERH_global_equations

Pspec generated 4 September 1990 at 15:00:50

This process has 3 data flows:

input dala flows

ERH_algorithm_controls

ERH_global_equations

output data flows

delta_potential

description

Factor ERH_global_equations

Solve factored linear system

Return delta_potential

end pspec 
Process 3.3.2.1

\section{ERH_element_equations_executive}

Process 3.3.2.1: ERH_element_equations_executive

Pspec generated 4 September 1990 at 15:02:07

This process has 6 data flows:

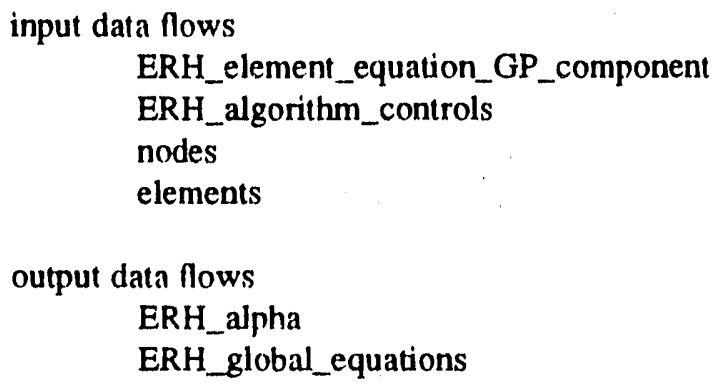

Foreach element

Foreach element node \{

Foreach Gauss_point I

compute shape function and derivative values compute Gauss point potential compute Gauss point electrical conductivity

apply integration rule to compute local element equation

1

1

accrmulate local element nodal equation into global equations

end pspec 
Process 3.3.2.2

\section{compute_ERH_element_shape_function_GP_values}

Process 3.3.2.2: compute_ERH_element_shape_function_GP_values

Pspec generated 4 September 1990 at 15:02:01

This process has 3 data flows:

input data flows

3D_canonical_functions

nodal_coordinates

output data flows

3D_ERH_element

description

compute determinant of transformation

evaluate shape functions at specified point

evaluate derivative functions at specified point

end pspec 
Process 3.3.2.3

compute_ERH_element GP_potential

Process 3.3.2.3: compute_ERH_element_GP_potential

Pspec generated 4 September 1990 at 15:02:11

This process has 3 data flows:

input dala flows

electrical_potential

fcn

output data flows

GP_potential

description

Foreach node 1

accumulate nodal potential times shape function value 1

end pspec 
Process 3.3.2.4

\section{compute_ERH_element_GP_electrical_conductivity}

Process 3.3.2.4: compute_ERH_element_GP_electrical_conductivity

Pspec generated 4 September 1990 at 15:02:04

This process has 5 data flows:

input data flows

element_composition

electrical_conductivity

GP_potential

temperature

output data flows

GP_electrical_conductivity

description

evaluate electrical conductivity correlation

end pspec 
Pricess 3.3.2.5

accumulate_ERH_element_equation_GP_component

Process 3.3.2.5: accumulate_ERH_element_equation_GP_component

Pspec generated 4 September 1990 at 15:02:14

This process has 5 data flows:

input data flows

GP_electrical_conductivity

ERH_alpha

electrical_potential

3D_ERH_element

output data flows

ERH_element_equation_GP_component

description

Scale GP_electrical_conductivity by one-eighth element volume (det)

Compute local stiffness matrix from derv and GP_electrical_conductivity

end pspec 
Process 3.3.3.1

\section{flux_boundary_executive}

Process 3.3.3.1: flux_boundary_executive

Pspec generated 4 September 1990 at 15:02:52

This process has 8 data flows:

input data flows

nodes

elements

Neumann_boundaries

boundary_integral_GP_component

ERH_Neumann_boundary_index

ERH_algorithm_controls

ERH_global_equations

output data flows

ERH_alpha

ERH_global_equations

description

Foreach surface element in the boundary 1

Foreach surface element node |

Foreach 2D_Gauss_point 1

compute all shape function values

compute Gauss current density

accumulate boundary integral GP component

1

1

apply integration rule to compute contribution to local equation

accumulate local element nodal equation into global equations

1

end pspec 
Process 3.3.3.2

compute_flux_element_shape_function_GP_values

Process 3.3.3.2: compute_flux_element_shape_function_GP_vulues

Pspec generated 4 September 1990 at 15:02:59

This process has 3 data flows:

input data flows

2D_canonical_functions

nodal_coordinates

output data flows

2D_ERH_flux_element

description

transform canonical element to physical element

calculate function values at specified point

calculate dis

end pspec 
Process 3.3.3.3

\section{compute_ERH_._surface_element_GP_current_density}

Process 3.3.3.3: compute_ERH_surface_element_GP_current_density

Pspec generated 4 September 1990 at 15:03:03

This process has 3 data flows:

input data flows

current_density_boundary_condition

func

output data flows

GP_current_density

description

Foreach component of current density \{

Foreach node of element 1

accumulate current density component times function value 1

1

end pspec 


\section{Process 3.3.3.4}

\section{accumulate_flux_boundary_Integral_GP_component}

Process 3.3.3.4: accumulate_flux_boundary_integral_GP_component

Pspec generated 4 Septembir 1990 at 15:03:08

This process has 4 data flows:

input data flows

ERH_alpha

GP_current_density

2D_ERH_flux_element

output data flows

boundary_integral_GP_component

description

scale GP_current density by dis

compute GP contribution to each nodal equation

end pspec 
Process 3.3,4.1

electrode_position_boundary_executive

Process 3.3.4,1: electrode_position_boundary_executive

Pspec generated 4 September 1990 at 15:03:51

This process has 7 dala flows:

input data flows

nodal_electrode_potential_boundary_value

ERH_Dirichlet_boundary_index

nodes

Dirichlet_boundaries

ERH_algorithm_controls

electrode_position

ERH_global_equations

outpul data flows

ERH_global_equations

description

Foreach node from top node to bottom node of electrode position 1

Get nodal electrode potential boundary value

Define nodal equation

1

Add penalty term to global equation to fix electrode potential value

end pspec 
Process 3.3.4.2

get_nodal_electrode_potential_boundary_value

Process 3.3.4.2: get_nodal_electrode_potential_boundary_value

Pspec generated 4 September 1990 at 15:03:47

This process has 2 data flows:

input data flows

electrode_boundary_condition

output data flows

nodal_electrode_potential_boundary_value

description

Evaluate electrode potential boundary value correlation

end pspec 
Procens 3.3.5.1

\section{potential_boundary_executive}

Process 3.3 .5 .1 : potential_boundary_executive

Pspec generated 4 September 1990 at 15:04:30

This process has 6 data flows:

input data flows

nodal_potential_boundary_value

ERH_Dirichlet_boundary_index

nodes

Dirichlet_boundaries

ERH_algorithm_controls

ERH_global_equations

output data flows

ERH_global_equations

description

Foreach node in Dirichlet boundary 1

Get nodal potential boundary value

Define nodal equation

1

Add penalty term to global equation to fix potential value

end pspec 
Process 3.3.5.2

get_nodal_potential_boundary_value

Process 3.3.5.2: get_nodal_potential_boundary_value

Pspec generated 4 September 1990 at 15:04:27

This process has 2 data flows:

input data flows

potential_boundary_condition

output data fiows

nodal_potential_boundary_value

description

Evaluate potential boundary value correlation

end pspec 
Process 3.3.7.1

\section{ERH_element_current_density_executive}

Process 3.3.7.1: ERH_element_current_density_executive

Pspec generated 5 September 1990 at 14:27:02

This process has 7 data flows:

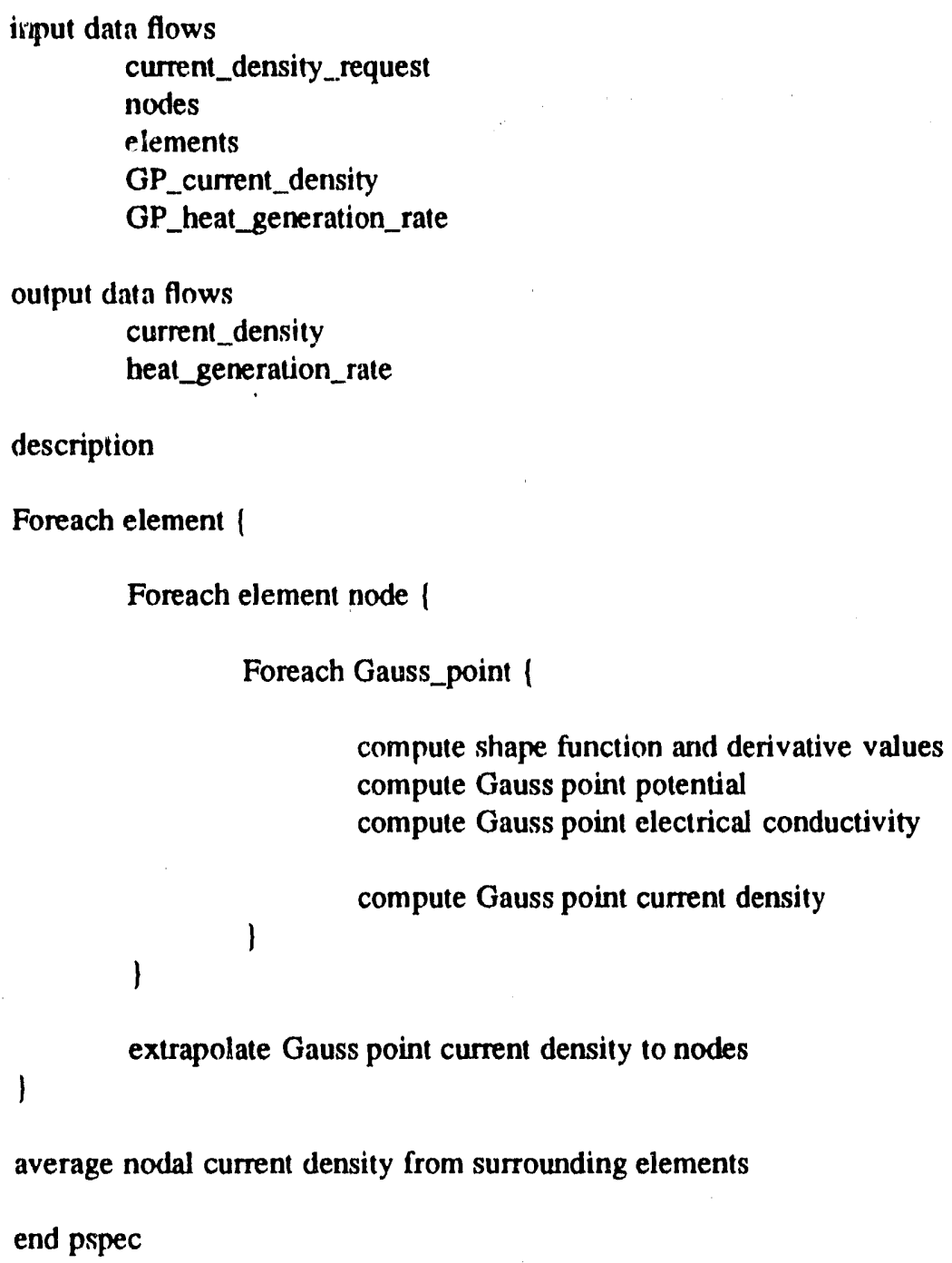


Process 3.3.7.2

\section{recompute_ERH_element_shape_function_GP_values}

Process 3.3.7.2: recompute_ERH_element_shape_function_GP_values

Pspec generated 5 September 1990 at 14:26:53

This process has 3 data flows:

input data flows

3D_canonical_functions

nodal_coordinates

output data flows

3D_ERH_flux_element

description

compute determinant of transformation

evaluate shape functions at specified point

evaluate derivative functions at specified point

end pspec 
Process 3.3.7.3

recompute_ERH_element_GP_potential

Process 3.3.7.3: recompute_ERH_element_GP_potential

Pspec generated 5 September 1990 at 14:27:07

This process has 3 data flows:

input dara flows

electrical_potential

fen

output data flows

GP_potential

description

Foreach node 1

accumulate nodal potential times shape function value

1

end pspec 
Process 3.3.7.4

compute_ERH_element_GP_potential_gradient

Process 3.3.7.4: compute_ERH_element_GP_potential_gradient

Pspec generated 5 September 1990 at 14:27:15

This process has 3 data flows:

input data flows

derv

electrical_potential

output data flows

GP_potential_gradient

description

Foreach component direction 1

Foreach node of element I

accumulate potential times component derivative value

1

1

end pspec 
Process 3.3.7.5

recompute_ERH_element_GP_electrical_conductivity

Process 3.3.7.5: recompute_ERH_element_GP_electrical_conductivity

Pspec generated 5 September 1990 at 14:26:58

This process has 5 data flows:

input data flows

electrical_conductivity

element_composition

GP_potential

temperature

output data flows

GP_electrical_conductivity

description

evalute electrical conductivity correlation

end pspec 
Process 3.3.7.6

compute_ERH_element_GP_current_density

Process 3.3.7.6: compute_ERH_element_GP_current_density

Pspec generated 5 September 1990 at 14:27:11

This process has 3 data flows:

input data flows

GP_electrical_conductivity

GP_potential_gradient

output data flows

GP_current_density

description

compute GP_electrical_conductivity times GP_temp_grad

end pspec 
Process 3.3.7.7

\section{compute_ERH_element_GP_heat_generation_rate}

Process 3.3.7.7: compute_ERH_element_GP_heat_generation_rate

Pspec genernted 5 September 1990 at 14:27:18

This process has 3 data flows:

input data flows

$$
\begin{aligned}
& \text { GP_current_density } \\
& \text { GP_potential_gradient }
\end{aligned}
$$

output data flows

GP_heat_generation_rate

description

compute GP_current_density times GP_potential_gradient

end pspec 


\section{Process 3.4.1}

\section{TET_executive}

Process 3.4.1: TET_executive

Pspec generated 4 September 1990 at 15:06:06

This process has 12 data flows:

input data flows

TET_controls

delta_temperature

output data flows

heat_flux_request

TET_messages

TET_algorithm_controls

temperature

electrode_position

time

TET_-algorithm_controls

TET_algorithm_controls

TET_algorithm_controls

TET_algorithm_controls

description

Repeat 1

Repeat 1

set trial_time to time plus duala_time

Repeat 1

form_TET_global_equations |

assemble TET element equations

apply convection boundary condition

apply radiation boundary condition

1

apply temperature boundary condition

solve_TET_global_equations

| Until ( TET_iteration_limit or TET_relative_tolerance )

If ( not converged or temperature change exceeds limit) then

If ( delta_time $<=\min \_\mathrm{dt}$ ) then

terminate TET problem

endif

reduce delta_time

endif

| Until ( converged and temperature change within limit )

set time to trial_time

adjust delta_time

update temperature values

update electrode position

| Until ( time equals 'TET_final_time ) 
compule_heat_flux

end pspec

B - 38 
Process 3.4.6

solve_TET_global_equations

Process 3.4.6: solve_TET_global_equations

Pspec generated 4 September 1990 at 15:06:01

This process has 3 data flows:

input data flows

TET_algorithm_controls

TET_global_equations

output data flows

delta_temperature

description

Factor TET_global_equations

Solve factored linear system

Return delta_temperature

end pspec 
Process 3.4.2.1

\section{TET_element_equations_executive}

Process 3.4.2.1: TET_element_equations_executive

Pspec generated 5 September 1990 at 14:33:40

This process has 7 data flows:

input data flows

TET_element_equation_GP_component

TET_algorithm_controls

nodes

elements

output data flows

delta_time

TET_alpha

TET_global_equations

description

Foreach element ।

Foreach element node 1

Foreach Gauss_point !

compute shape function and derivative values

collpute Gauss poinst temperature

compute Gauss point thermal quantities

compute Gauss point ERH heat generation rate

compute Gauss point heat generation rate

accumulate local element equation GP component

1

apply integration rule to compute local element equation

accumulate local element nodal equation into global equations

end pspec 
Process 3.4.2.2

compute_TET_element_shape_function_GP_values

Process 3.4.2.2: compute_TET_element_shape_function_GP_values

Pspec generated 5 September 1990 at 14:33:36

This process has 3 duta flows:

input data flows

3D_canonical_functions

nodal_coordinates

output datn flows

3D_TET_element

description

compute determinant of transformation

evaluate shape functions at specified point

evaluate derivative functions at specified point

end pspec 
Process 3.4.2.3

compute_TET_element_GP_temperature

Process 3.4.2.3: compute_TET_element_GP_temperature

Pspec generated 5 September 1990 at 14:33:44

This process has 3 data flows:

input data flows

temperature

$\mathrm{fcn}$

output data flows

GP_temperature

description

Foreach node 1

accumulate nodal temperature times shape function value

1

end pspec 
Process 3.4.2.4

\section{compute_TET_element_GP_ER_H_heat_generation_rate}

Process 3.4.2.4: compute_TET_element_G ERH_heat_generation_rate

Pspec generated 5 September 1990 at 14:33:54

This process has 3 data flows:

input data flows

fon

heat_generation_rate

output data flows

GP_ERH_heat_generation_rate

description

Foreach node of element I

accumulate nodal heat generation_rate component times fon value 1

end pspec 
Process 3.4.2.6

compute_TET_element_GP_heat_generation_rate

Process 3.4.2.6: compute_TET_element_GP_heat_generation_rate

Pspec generated 5 September 1990 at 14:33:51

This process has 5 data flows:

input data flows

delta_time

GP_phase_change_energy

GP_electrical_conductivity

GP_ERH_heat_generation_rate

output data fiows

GP_heat_generation_rate

description

compute phase change energy rate

sum ERH heat generation rate and phase change energy rate

end pspec 
Process 3.4.2.7

accumulate_TET_element_equation_GP_component

Process 3.4.2.7: accumulate_TET_element_equation_GP_component

Pspec generaled 5 september 1990 at 14:33:47

This process has 8 data flows:

input data flows

GP_density_heat_capacity

GP_heat_generation_rate

TET_alpha

delta_time

GP_thermal_conductivity

temperature

3D_TET_element

output data flows

TET_element_equation_GP_component

description

Scale GP_thermal_conductivity by one-eighth element volume (det)

Scale GP_density_heat_capacity by one-eighth element volume

Scale GP_heat_generation_rate by one-eighth element volume

Compute local stiffness matrix as function of derv and GP_thermal_conductivity

Compute local ths as function of $\mathrm{fcn}$ and $\mathrm{GP}$ _heal_generation_rate

Compute local mass matrix as function of fen and GP_density_heal_capacity

Perform diagonal lumping of mass matrix by row sum

Add stiffness matrix contribution to ths

Add stiffness and mass matrix contributions

end pspec 
Process 3.4.2.5.1

\section{compute_TET_element_GP_thermal_conductivity}

Process 3.4.2.5.1: compute_TET_element_GP_thermal_conductivity

Pspec generated 4 September 1990 at 15:08:09

This process has 4 data llows:

input data flows

GP_temperature

element_composition

thermal_conductivity

output data flows

GP_thermal_conductivity

description

evaluate thermal conductivity correlation

end pspec 
Process 3.4.2.5.2

\section{compute_TET_element_GP_density_heat_capacity}

Process 3.4.2.5.2: compute_TET_element_GP_density_heat_capacity

Pspec generated 4 Seplember 1990 at 15:08:13

This process has 6 data flows:

input data flows

heat_capacity

GP_temperature

density

element_composition

output data flows

GP_density_heat_capacity

GP_heat_capacity

description

evaluate density correlation

evaluate heat capacity correlation

compute density and heal_capacity product

end pspec 


\section{Process 3.4.2.5.3}

\section{compute_TET_element_GP_phase_change_energy}

Process 3.4.2.5.3: compute_TET_elemient_GP_phase_change_energy

Pspec generated 4 September 1990 at 15:08:15

This process has 10 data flows:

inpul data flows

heat_of_fusion

melting_point

GP_temperature

solid_fraction

GP_heat_capacity

density

element_composition

boiling..point

heat_of_vaporization

output data flows

GP_phase_change_energy

description

if ( GP_temp passes through melting_point ) then compute fraction melted

endif compute fusion energy

if ( GP_temp passes through boiling_point ) then compute fraction vaporized

endif compute vaporization energy

add fusion energy to vaporization energy for phase change energy

end pspec 
Process 3.4.2.5.4

\section{compute_TET_element_GP_electrical_conducivity}

Process 3.4.2.5.4: compute_TET_element_GP_electrical_conducivity

Pspec generated 4 Septembe: 1990 at 15:08:19

This process has 4 data lows:

input data flows

electrical_conductivity

GP_temperature

element_composition

output data flows

GP_electrical_conductivity

description

evaluate electrical conductivity correlation

end pspec 
Process 3.4.3.1

convection_boundary_executive

Process 3.4.3.1: convection_boundary_executive

Pspec generated 4 September 1990 at 15:09:01

This process has 8 data flows:

input data flows

nodes

elements

Neumann_boundaries

boundary_integral_GP_component

TET_Neumann_boundary_index

TET_algorithm_controls

TET_global_equations

output data flows

TET_alpha

TET_global_equations

description

Foreach surface element in the boundary 1

Get nodal convection heat transfer coefficients

Foreach surface element node 1

Foreach 2D_Gauss_point 1

compute all shape function values compute Gauss noint temperature compute Gauss point convecison heat transfer coef accumulate boundary integral Gl' component

1

1

apply integration rule to compute contribution to local equation

accumulate local element nodal equation into global equations

1

end pspec 
Process 3.4.3.2

compute_nodal_chtc

Process 3.4.3.2: compule_nodal_chtc

Pspec generated 4 September 1990 at 15:08:58

This process has 3 data flows:

input data flows

convection_boundary_condition

temiperature

output data flows

nodal_chtc

description

evaluate chtc coefficient correlation

end pspec 
Process 3.4.3.3

\section{compute_convection_element_shape_function_GP_values}

Process 3.4 .3 .3 : compute_convection_element_shape_function_GP_values

Pspec generated 4 September 1990 at 15:09:06

This process has 3 data flows:

input data flows

2D_canonical_functions

nodal_coordinates

output data flows

2D_TET_convection_element

description

calculate function values at specified point

calculate dis

end pspec 
Process 3.4.3.4

\section{compute_convection_element_GP_temperature}

Process 3.4.3.4: compute_convection_element_GP_temperature

Pspec generated 4 September 1990 at 15:09:10

Thits process has 3 data flows:

input data flows

temperature

func

output data flows

GP_temperature

description

Foreach node of element 1

accumulate temperature times function value

1

end pspec 
Process 3.4.3.5

compute_GP_chtc

Process 3.4.3.5: compute_GP_chtc

Pspec generated 4 September 1990 at 15:09:14

This process has 3 data flows:

input data flows

nodal_chic

func

output data flows

GP_chtc

description

Foreach node of element 1

accumulate chtc times function value 1

end pspec 


\section{Process 3,4.3.6}

accumulate_convection_boundary_integral_GP_component

Process 3.4.3.6: accumulate_convection_boundary_integral_GP_component

Pspec generated 4 September 1990 at 15:09:17

This process has 7 data flows:

input data flows

$$
\begin{aligned}
& \text { TET_alpha } \\
& \text { GP_chtc } \\
& \text { GP_temperature } \\
& \text { convection_air_temperature } \\
& \text { 2D_TET_convection_elenient }
\end{aligned}
$$

output data flows

boundary_integril_..GP_component

convection_energy

description

scale GP_chtc by dis

compute GP contribution to each nodal equation

end pspec 


\section{Process 3.4.4.1}

\section{radiation_boundary_executive}

Process 3,4.4,1: radiation_boundary_executive

Pspec generated 4 September 1990 at 15:10:00

This process has 8 data flows:

input data flows

nodes

elements

Neumann_boundaries

boundary_integral_GP_component

TET_Neunann_boundary_index

TET_algorithm_controls

TET_global_equations

output data flows

TET_alpha

TET_global_equations

description

Foreach surface element in the boundary 1

Get nodal radiation heat transfer coefficients

Foreach surface element node 1

Foreach 2D_Gauss_point I compute all shape function values compute Gauss point temperature compute Gauss point radiation heat transfer coef accumulate boundary integral GP component 


\section{Process 3.4.4.2}

compute_modal_rhtc

Process 3.4.4.2: compute_nodal_thtc

Pspec generated 4 September 1990 at 15:09:57

This process has 3 data flows:

input data flows

radiation_boundary_condition

temperature

output data flows

nodal_thtc

description

evaluate thtc coefficient correlation

end pspec 
Process 3.4.4.3

\section{compute_radiation_element_shape_function_GP_values}

Process 3.4.4.3: compute_radiation_element_shape_function_GP_valiues

Pspec generated 4 September 1990 at 15:10:05

This process has 3 data flows:

input data flows

2D_canonical_functions

nodal_coordinates

output data flows

2D_TET_radiation_element

description

calculare function values at specified point

calculate dis

end pspec 
Process 3.4.4.4

compute_radiation_element_GP_temperature

Process 3.4.4.4: compute_radiation_element_GP_temperature

Pspec generated 4 September 1990 at 15:10:08

This process has 3 data flows:

input data florys

temperature

func

output data flows

GP_temperature

description

Foreach node of element 1

accumulate temperature times function value 1

end pspec 
Process 3.4.4.5

compute_GP_rhtc

Process 3.4.4.5: compute_GP_rhtc

Pspec generated 4 September 1990 at 15:10:12

This process has 3 data flows:

input data flows

nodal_rhtc

func

output data flows

GP_rhtc

description

Foreach node of element 1

accumulate ith times function value

1

end pspec 
Process 3.4.4.6

\section{accumulate_radiation_boundary_integral_GP_component}

Process 3.4.4.6: accumulate_radiation_boundary_integral_GP_component

Pspec generated 4 September 1990 at 15:10:15

This process has 7 data flows:

input data flows

TET_alpha

GP_ritc

GP_temperature

radiation_air_temperature

2D_TET_radiation_element

output data flows

boundary_integral_GP_component

radiation_energy

description

scale GP_rhtc by dis

compute GP contribution to each nodal equation

end pspec 
Process 3.4.5.1

temperature_boundary_executive

Process 3.4.5.1: temperature_boundary_executive

Thpocigenerated 4 September 1990 at 15:10:57

Tuis throcess has 6 dala flows:

input data flows

nodal_temperature_houndary_value

T'ET_Dirichlet_boundary_index

nodes

Dirichlet_boundaries

TET_algorithm_controls

TET_global_equations

output data flows

TET_global_equations

description

Foreach node in Dirichlet boundary 1

Get nodal temperature boundary value

Define nodal equation

Add penalty term to global equation to fix temperature value

I

end pspec 
Process 3.4.5.2

\section{get_nodal_temperature_boundary_value}

Process 3.4.5.2: get_nodal_temperature_boundary_value

Pspec generated 4 September 1990 at 15:10:54

This process has 2 data flows:

input data flows

temperature_boundary_condition

output data flows

nodal_temperature_boundary_value

description

Evaluate temperature boundary value correlation

end pspec 
Process 3.4.7.1

TET_element_heat_flux_executive

Process 3.4.7.1: TET_element_heat_flux_executive

Pspec generated 4 September 1990 at 15:11:37

This process has 5 dala flows:

input data flows

heat_flux_request

nodes

elements

GP_heat_flux

outpul data flows

heat_flux

description

Foreach Gauss_point 1

Foreach element 1

Foreach element node 1

compute shape function and derivative values

compute Gauss point temperature

compute Gauss point thermal conductivity

compute Gauss point heat flux

1

extrapolate Gauss point heat flux to nodes

1

average nodul heat flux from surrounding elements

and pspec 
Process 3.4.7.2

\section{recompute_TET_element_shape_function_GP_values}

Process 3.4.7.2: recompute_TET_element_shape_function_GP_values

Pspec generaled 4 September 1990 at $15: 11: 31$

This process has 3 data flows:

input data flows

3D_canonical_functions

nodal_coordinates

output data flows

3D_TET_flux_element

description

compute determinant of transformation evaluate shape functions at specitied point

evaluate derivative functions at specified point

end pspec 


\section{Process 3.4.7.3}

\section{recompute_TET_element_GP_temperature}

Process 3.4.7.3: recompute_TET_element_GP_temperature

Pspec generated 4 September 1990 at 15:11:42

This process has 3 data flows:

input data flows

temperature

fcn

output data flows

GP_temperature

description

Foreach node 1

accumulate nodal temperature times shape function value

1

end pspec 
Process 3.4.7.4

\section{compute_TET_element_GP_temperature_gradient}

Process 3.4.7.4: compute_TET_element_GP_temperature_gradient

Pspec generated 4 September 1990 at 15:11:48

This process has 3 dala flows:

input data flows

derv

temperature

output data flows

GP_temperature_gradient

description

Foreach component direction 1

Foreach node of element ।

accumulate temperature times component derivative value

1

end pspec 
Process 3.4.7.5

\section{recompute_TET_element_GP_thermal_conductivity}

Process 3.4.7.5: recompute_TET_element_GP_thermal_conductivity

Pspec generated 4 September 1990 at 15:11:34

This process has 4 data flows:

input data flows

thermal_conductivity

element_composition

GP_temperature

output data flows

GP_thermal_conductivity

description

evalute thermal conductivity correlation

end pspec 
Process 3.4.7.6

compute_TET_element_GP_heat_Hux

Process 3.4.7.6: compute_TET_element_GP_heat_flux

Pspec generated 4 Septemher 1990 at 15:11:45

This process has 3 data flows:

input data flows

GP_thermal_..conductivity

GP_temperature_gradient

output data flows

GP_heat_flux

description

compute thermal conductivity times GP_temperature_gradient

end pspec 
Process 4.1

ISV_output_executive

Process 4.1: ISV_output_executive

Pspec genernted 4 September 1990 at 15:12:30

This process has 10 data flows:

input dita flows

oulput_menu_selection

output_responses

output_selection

solution_summary

display_attributes_file

output data flows

selected_.problem_name

output_prompts

output_menu

channel_name

display_attributes_file

attribute_buffer

description

Set load_problem into output_menu_selection

Do (forever) 1

Switch (output_ment__selection) | case load_problem:

Gel list of problem names

Display list of problem names

Prompt for selection

Get selected_.problem_name

Invoke load process

Set load_channels into output_menu_selection

break;

case load_channels:

Display channel names menu

Get chan_name_selection

Switch ( chan_name_selection ) I

case channel_name:

Pul channel_name in buffer

Display channel names menu

Get chan_name_selection

break:

case load_problem_request:

Set loud_protlem into output_menu_selection continue

break;

case display_request:

Set display_data into output_menu_selection

continue

breal ; 
Process 4.2

\section{load_general_problem_data}

Process 4.2: load_general_problem_data

Pspec generated 4 September 1990 at 15:12:39

This process has 3 data flows:

input data flows

selected_problem_name

xdr_binary_problem_file

output data flows

solution_summary

description

Open the problem file using selected_problem_name

Read time history information from problem file

Load time history information into solution summary

Read available data channel names for problem file

Load available data channel names into solution summary

end pspec 


\section{Process 4.3}

display_data_channels

Process 4.3: display_data_channels

Pspec generated 4 September 1990 at 15:12:43

This process has 7 data flows:

input datn flows

xdr_binary_geometry_file

xdr_binary_problem_file

channel_buffer

attribute_buffer

output dara flows

tabular_output

graphic_output

channel_data

description

Read data channel name(s) and display attributes

Load required problem data from problem file and geometry file

Produce the specified graphic or tabular output

end pspec 


\title{
APPENDIX C - DATA STRUCTURE DIAGRAMS
}

\author{
Diagram: 2D_physical_element \\ Diagram: 3D_correlation \\ Diagram: 3D_physical_element \\ Diagram: 3D_vector \\ Diagram: ERH_boundary_conditions \\ Diagram: FE_mesh \\ Diagram: FE_method \\ Diagram: GP_values \\ Diagram: ISV_executive_io \\ Diagram: TET_boundary_conditions \\ Diagram: boundary_variables \\ Diagram: correlation \\ Diagram: element_variables \\ Diagram: input_executive_io \\ Diagram: material_properties \\ Diagram: matrix_equation \\ Diagram: misc \\ Diagram: node_variables \\ Diagram: output_executive_io \\ Diagram: problem_controls \\ Diagram: problem_data \\ Diagram: simulation_executive_io
}



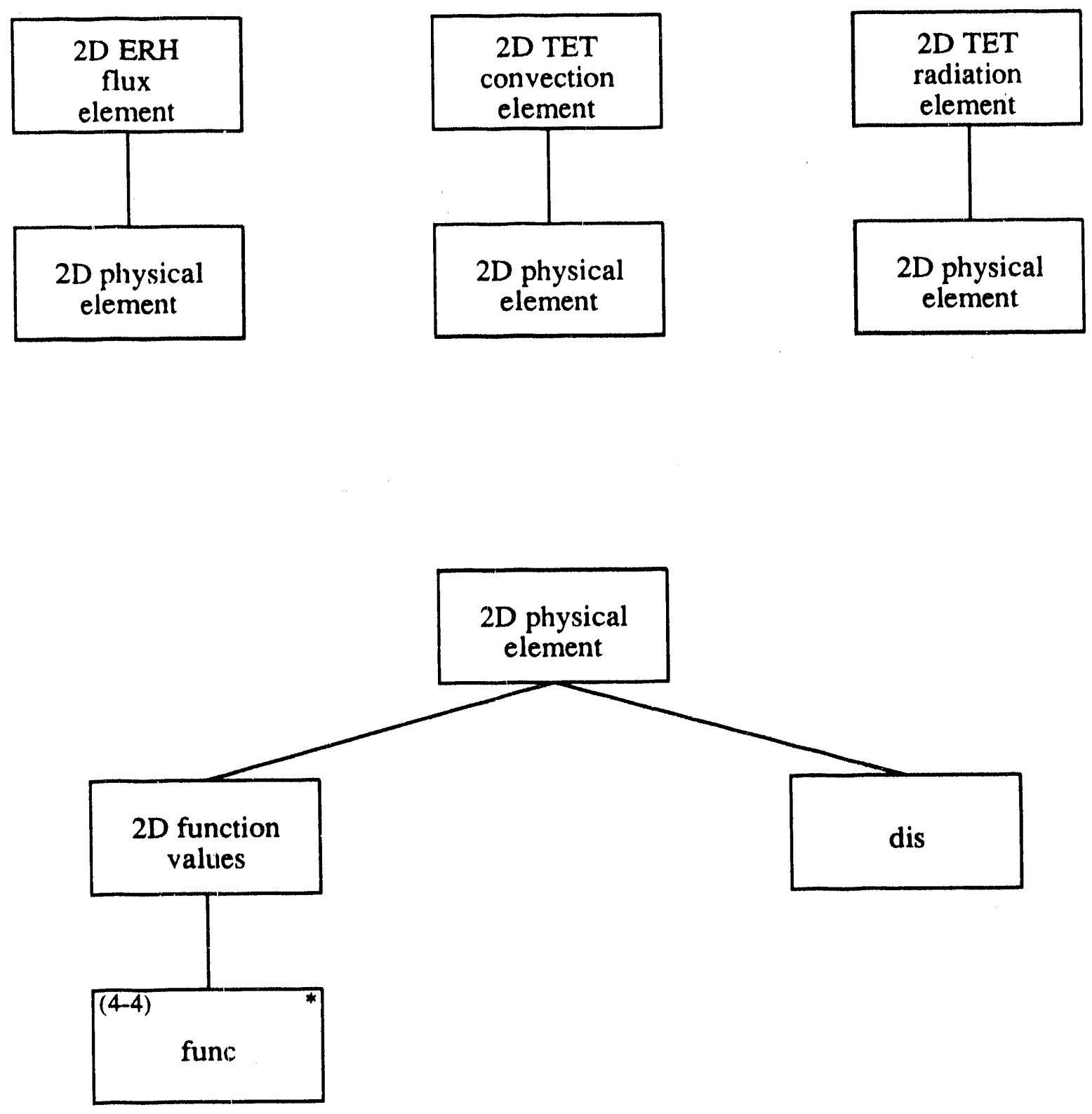
Project: In Situ Vitrification

Diagram: 3D_correlation

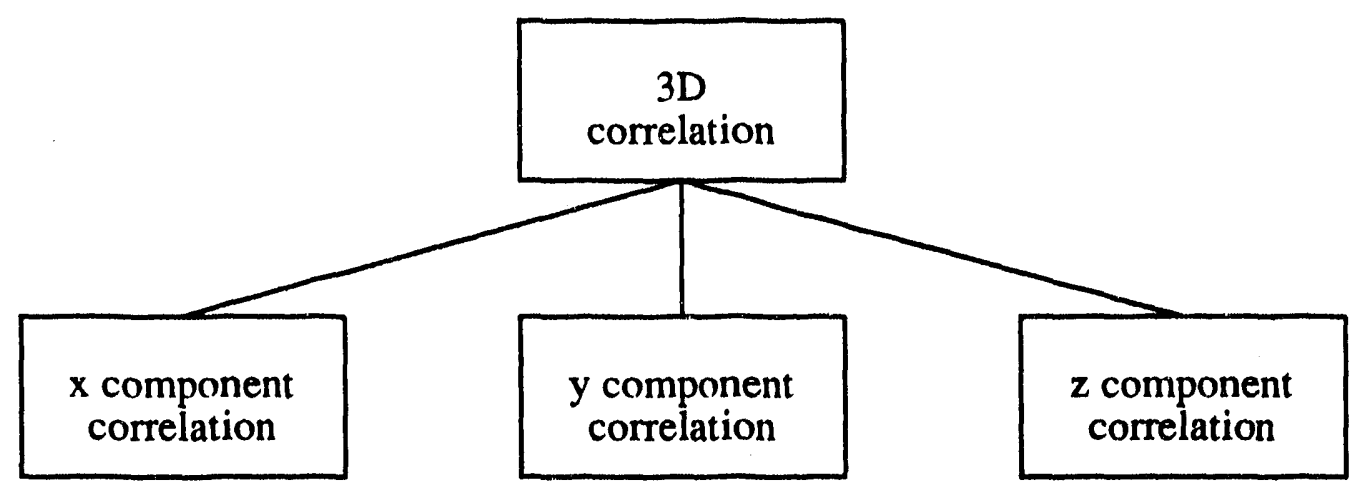

C - 3 
Project: In Situ Vitrification

Diagram: 3D_physical_element
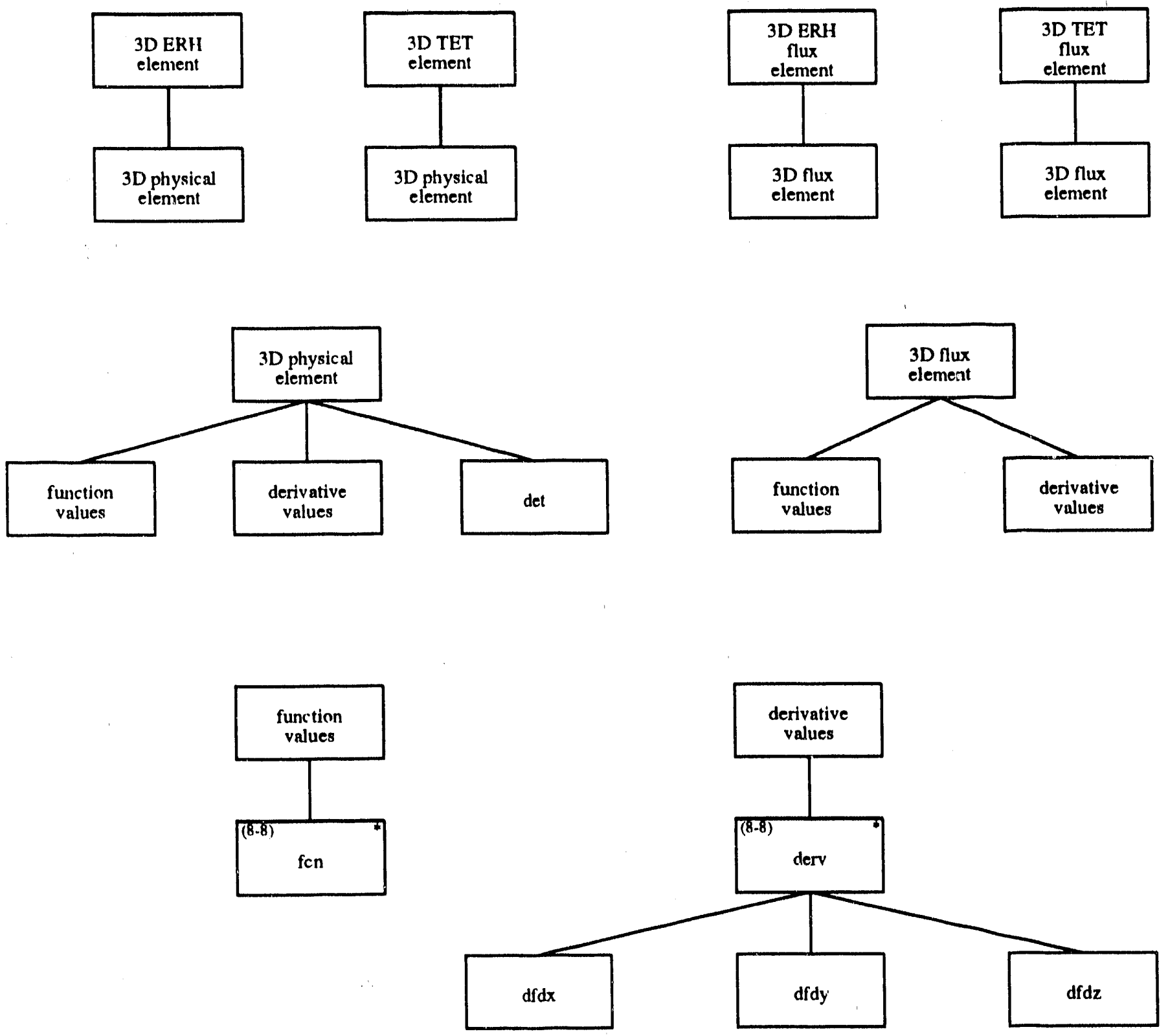
Project: In Situ Vitrification

Diagram: 3D_vector

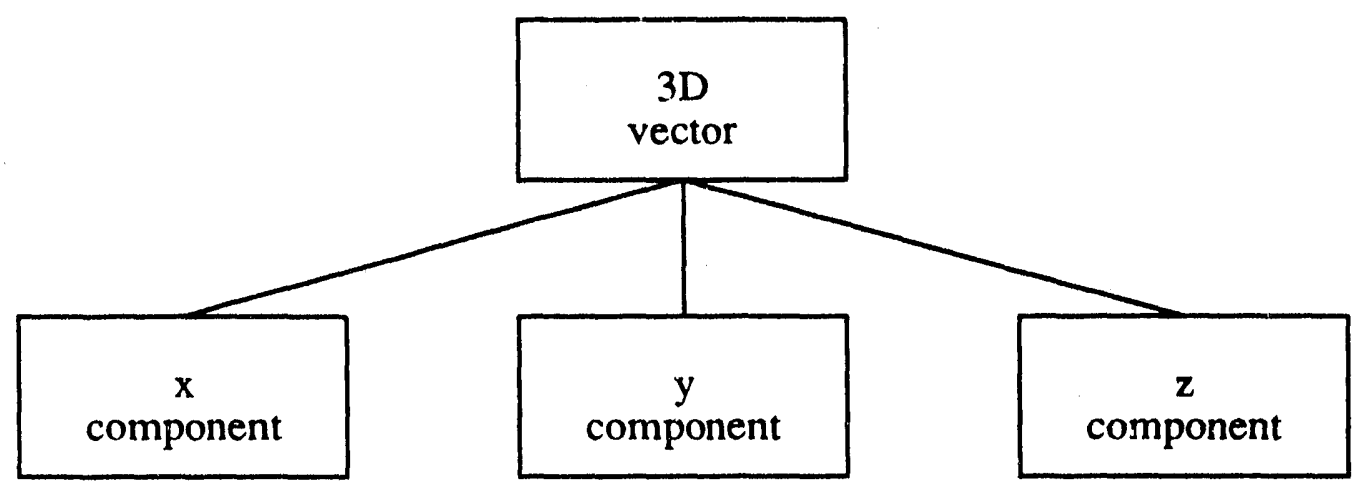

C - 5 
Project: In Situ Vitrification

Diagram: ERH_boundary_conditions

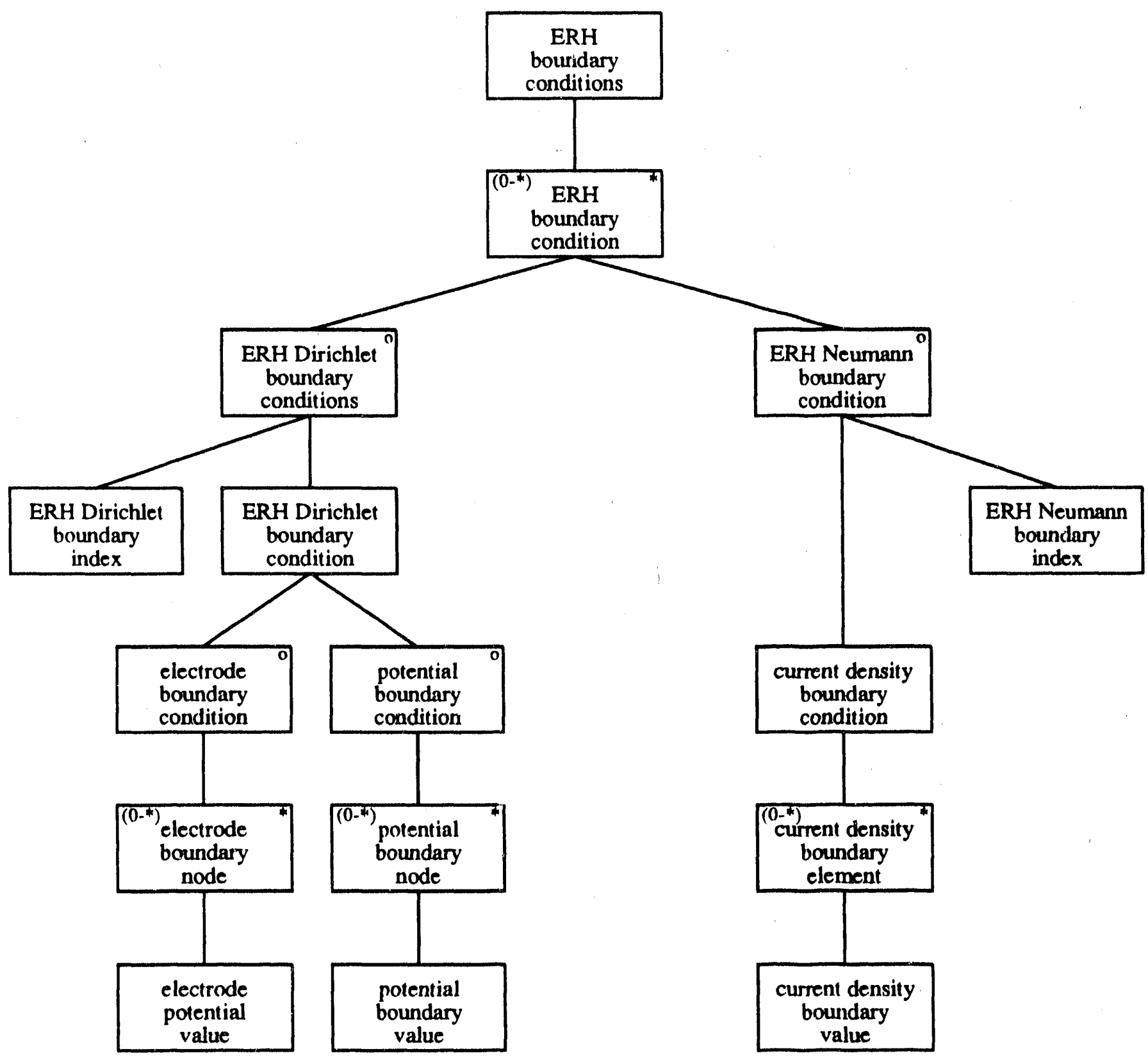

C. 6 
Project: In Situ Vitrification

Diagram: FE_mesh

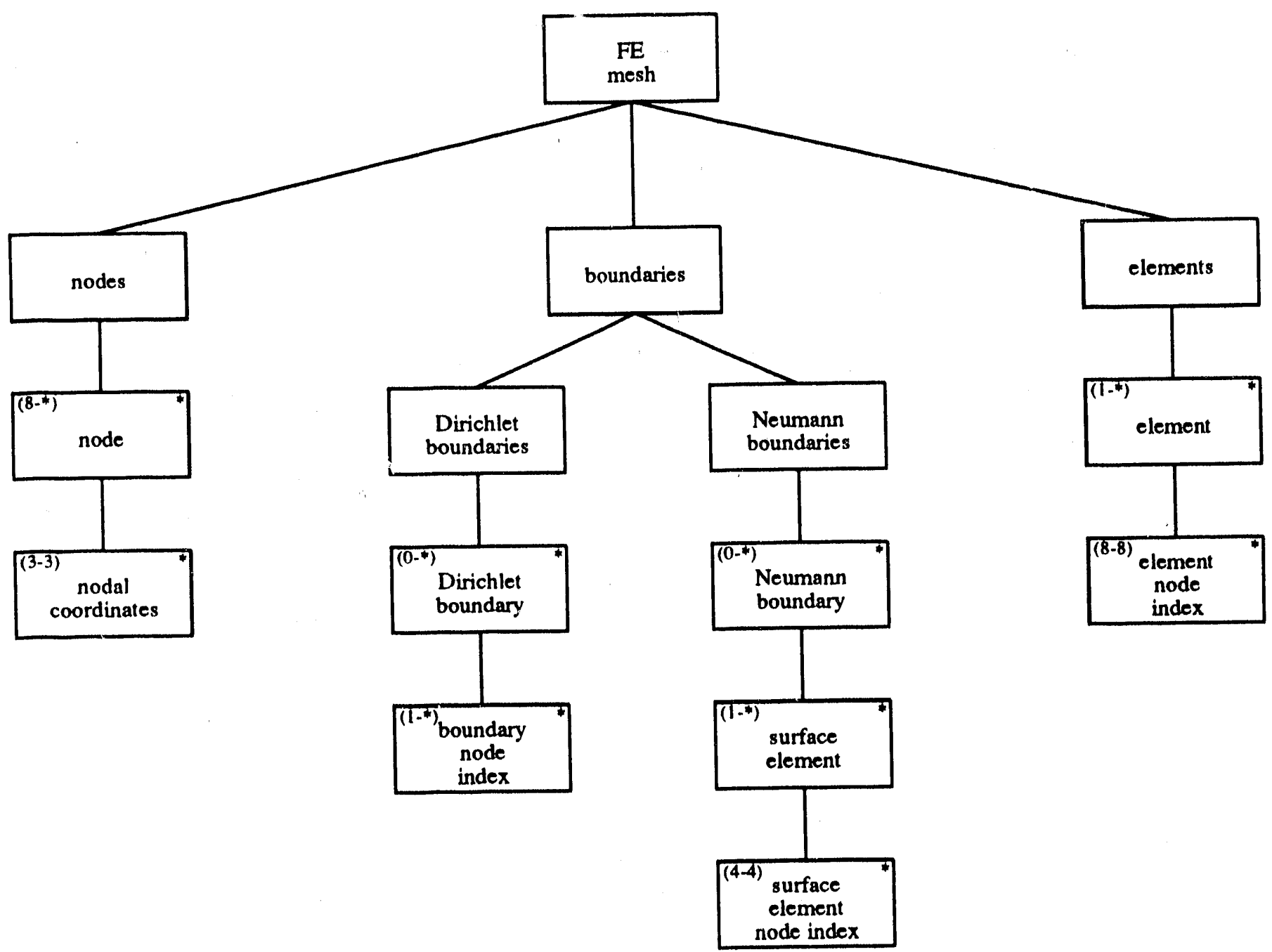


Project: In Situ Vitrification

Diagram: FE_method

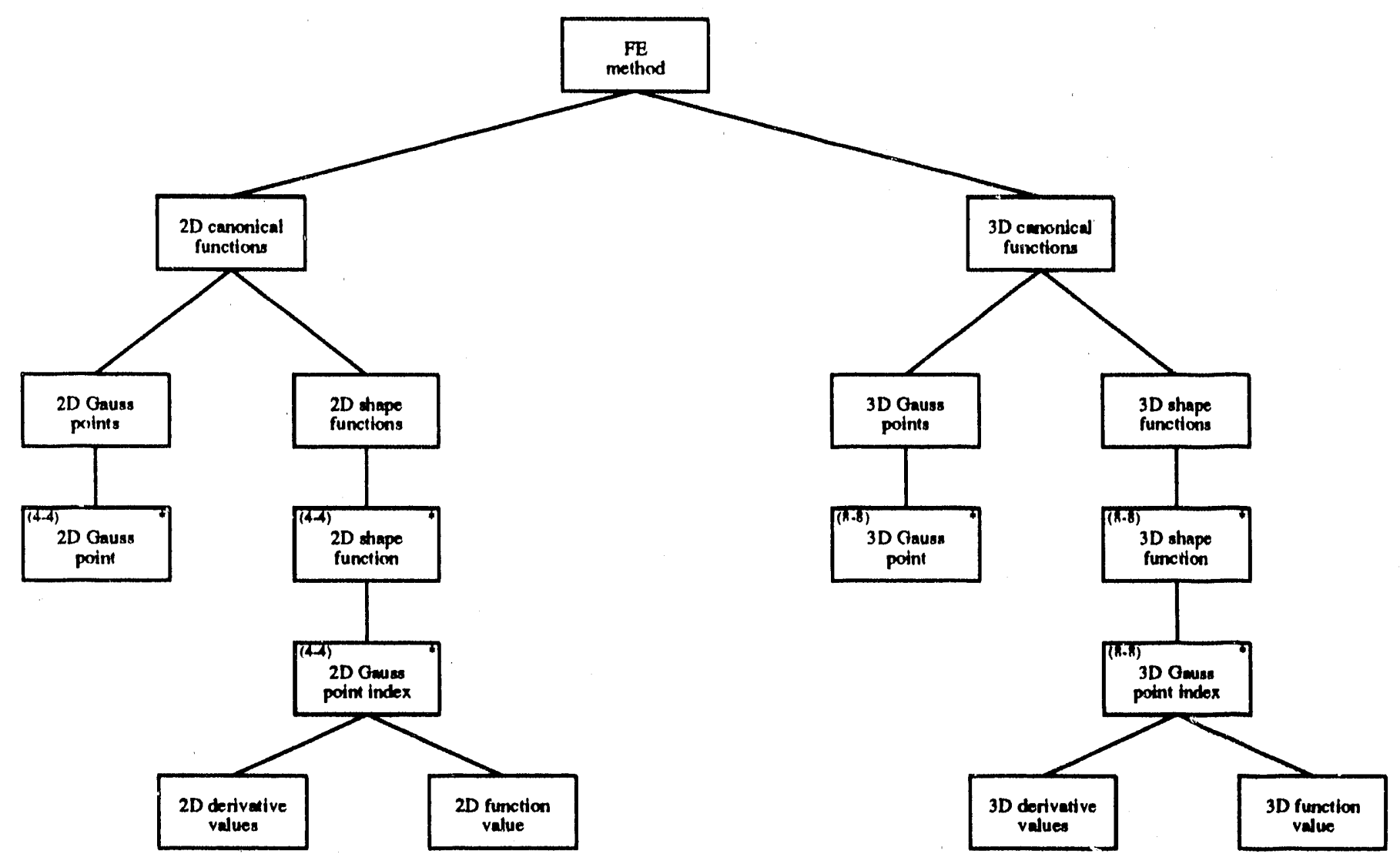


Project: In Situ Vitrification

Diagram: GP_values

GP

potential
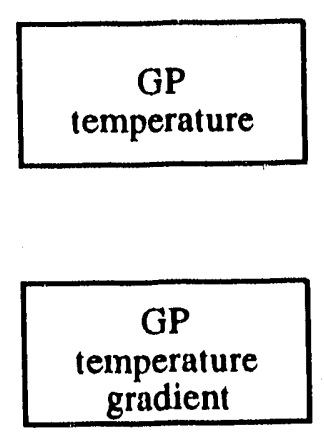

\section{GP ERH heat generation \\ rate}

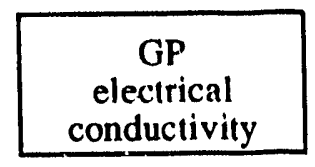

\section{ERH element \\ equation \\ GP component}

matrix

equation

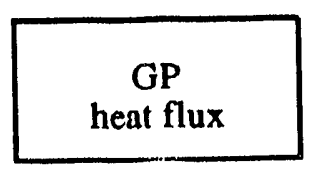

GP heat

generation rate

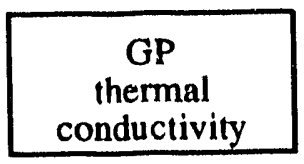

GP

phase change energy

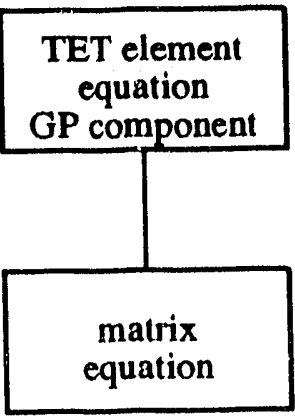

matrix

equation boundary integral

GP component

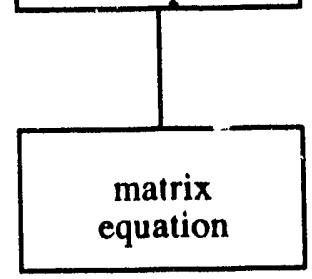

GP heat capacity

GP

heat capacity 
Project: In Situ Vitrification

Diagram: ISV_executive_io

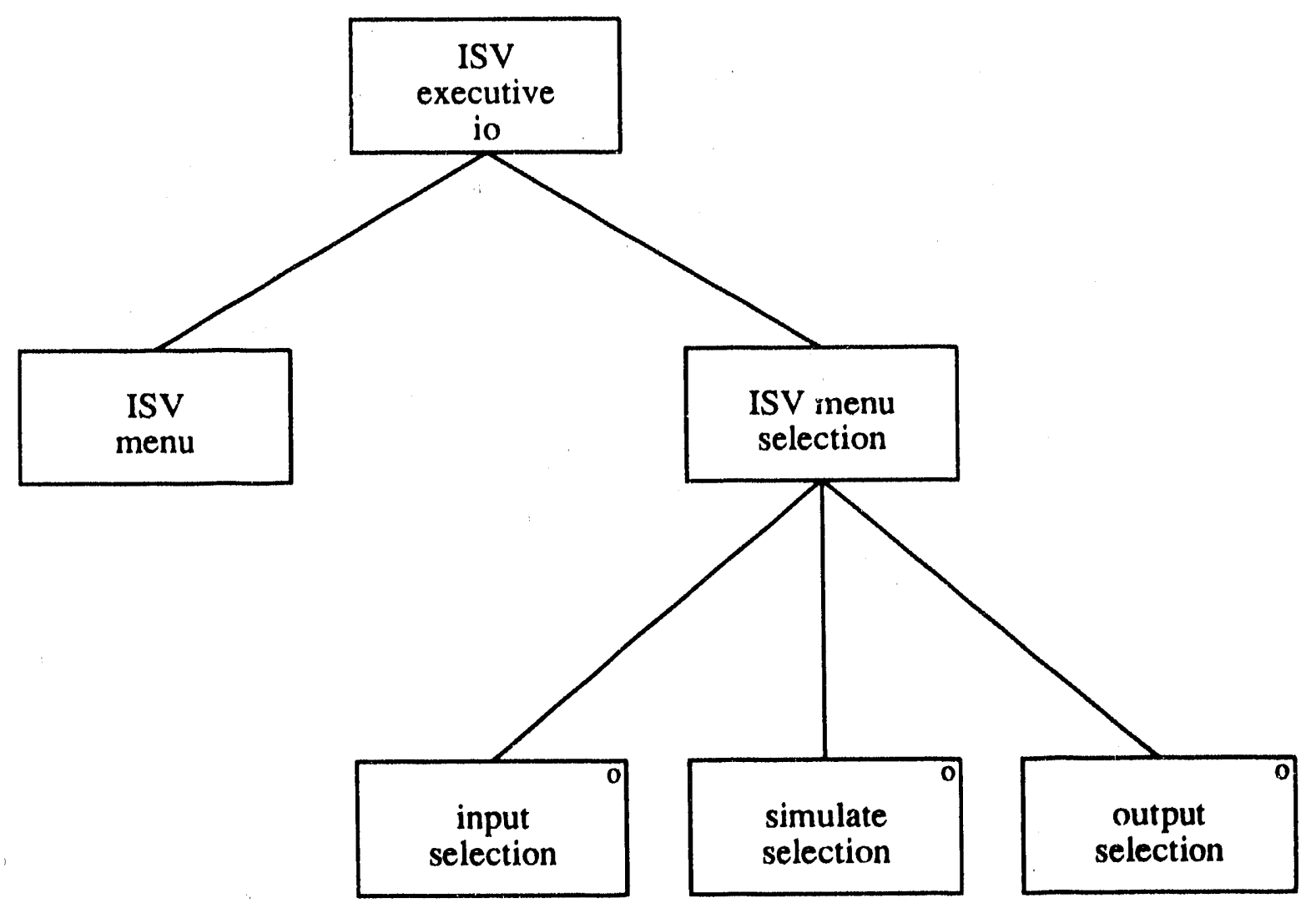


Project: In Situ Vitrification

Diagram: TET_boundary_conditions

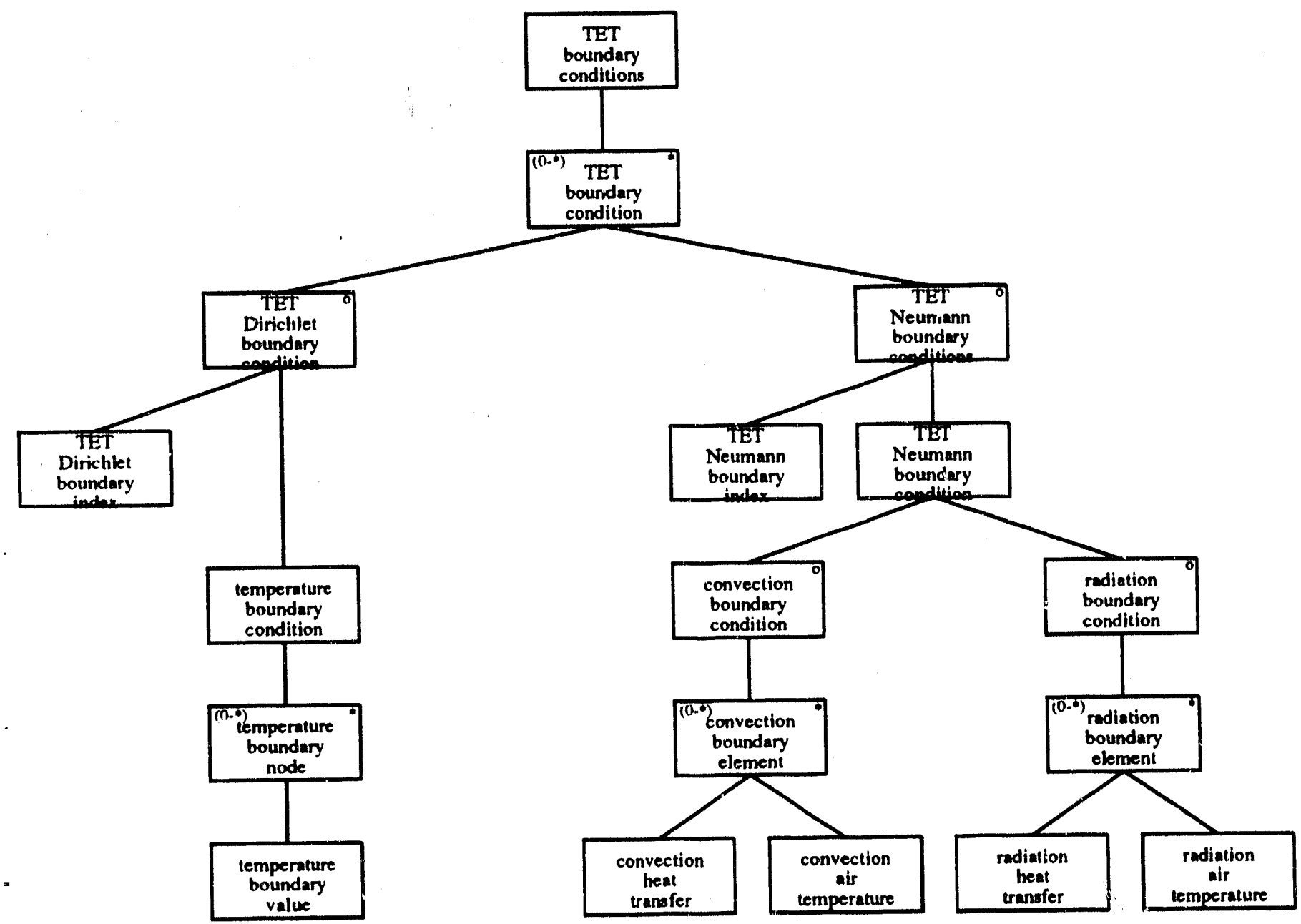




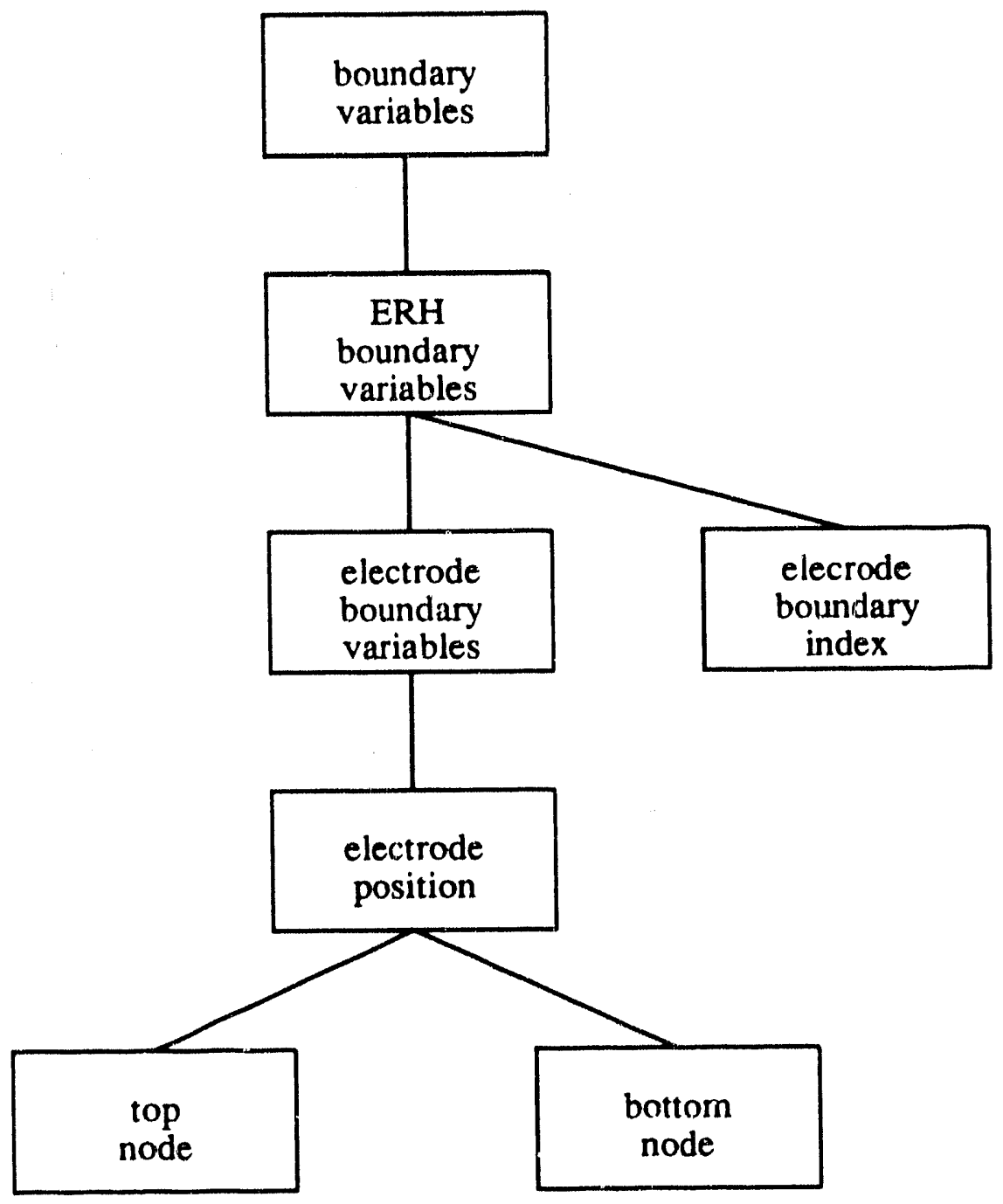


Project: In Situ Vitrification

Diagram: correlation

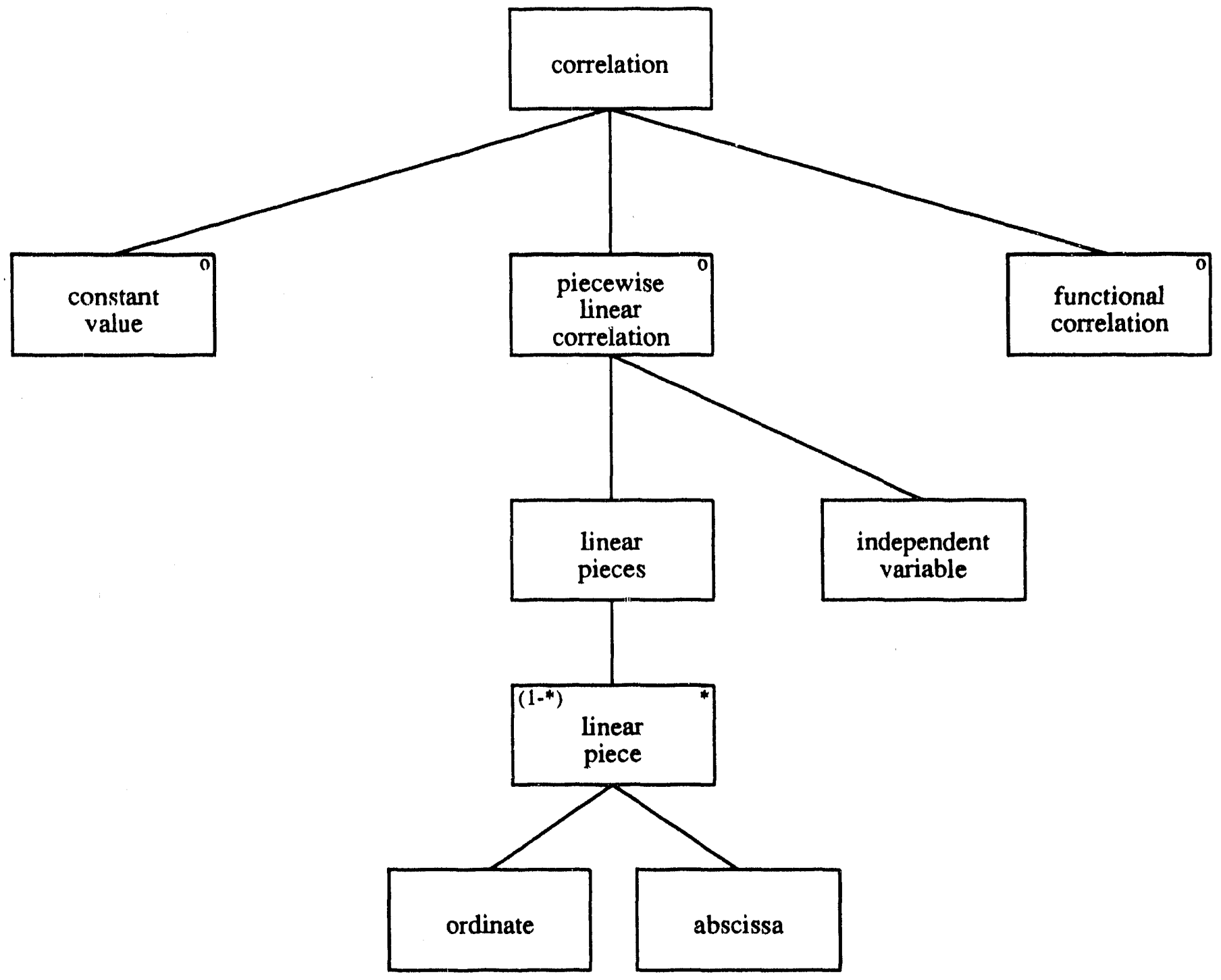


Project: In Situ Vitrification

Diagram: element_variables

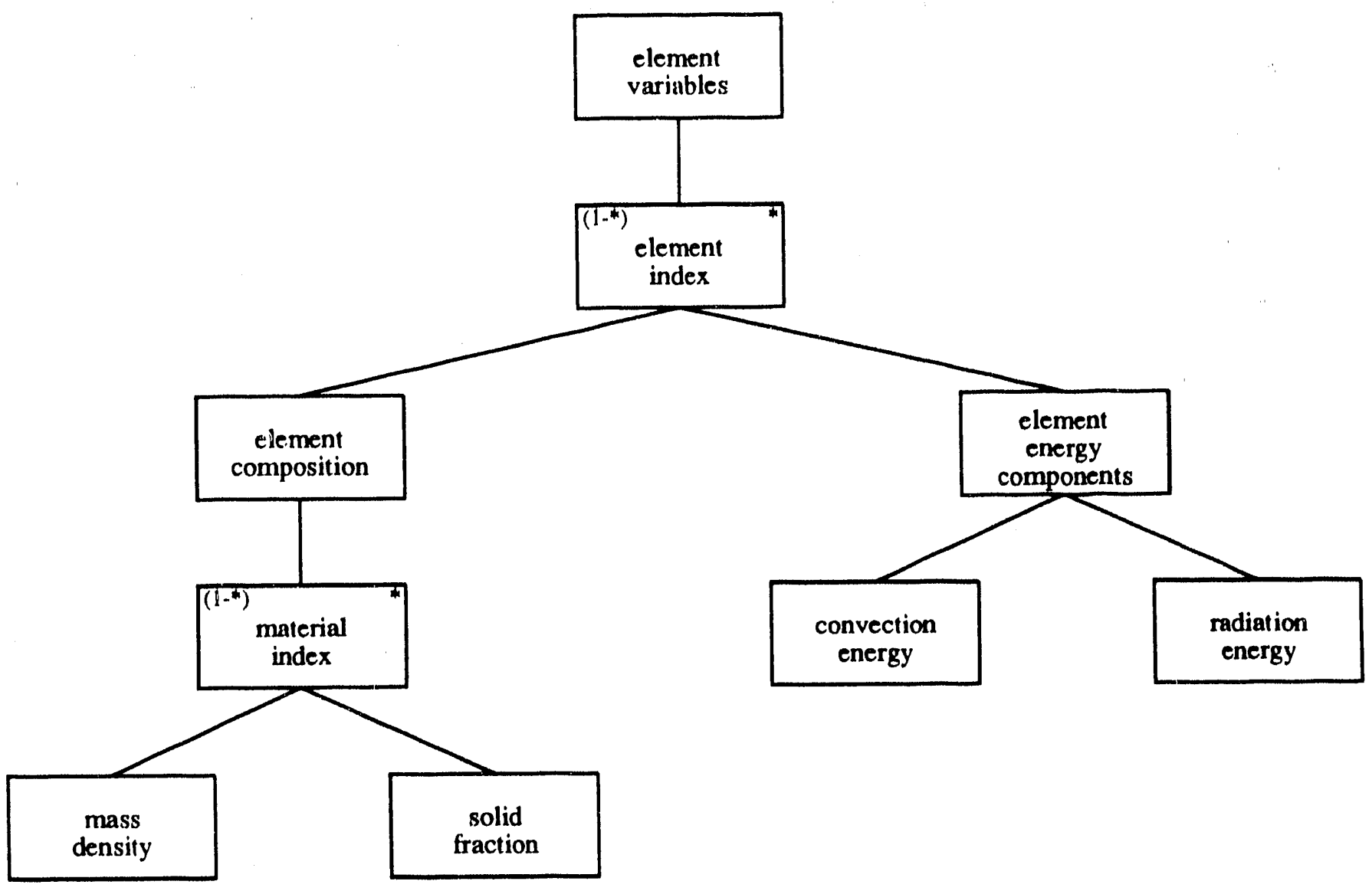


Project: In Situ Vitrification

Diagram: input_executive_io
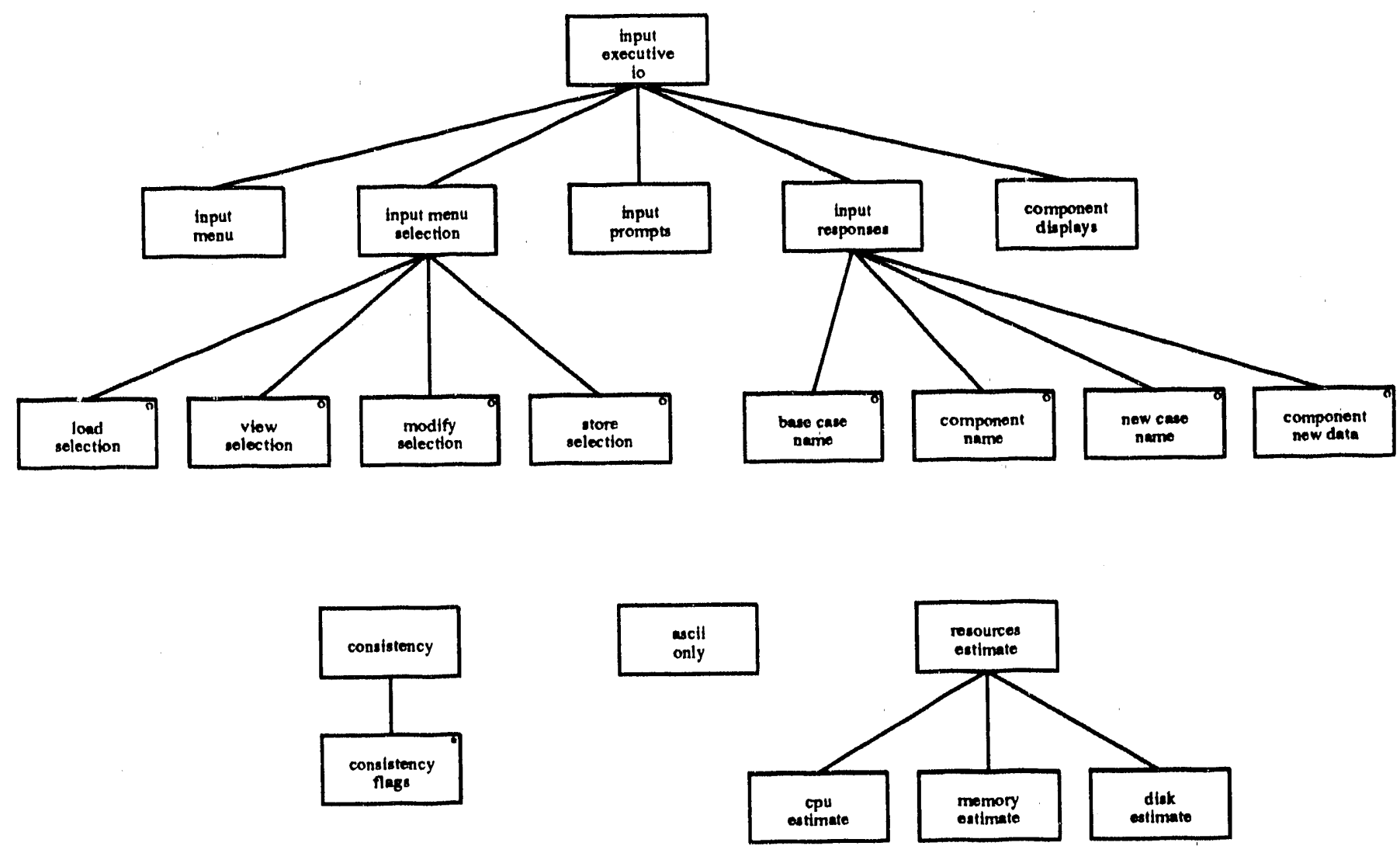
Project: In Situ Vitrification

Diagram: material_properties

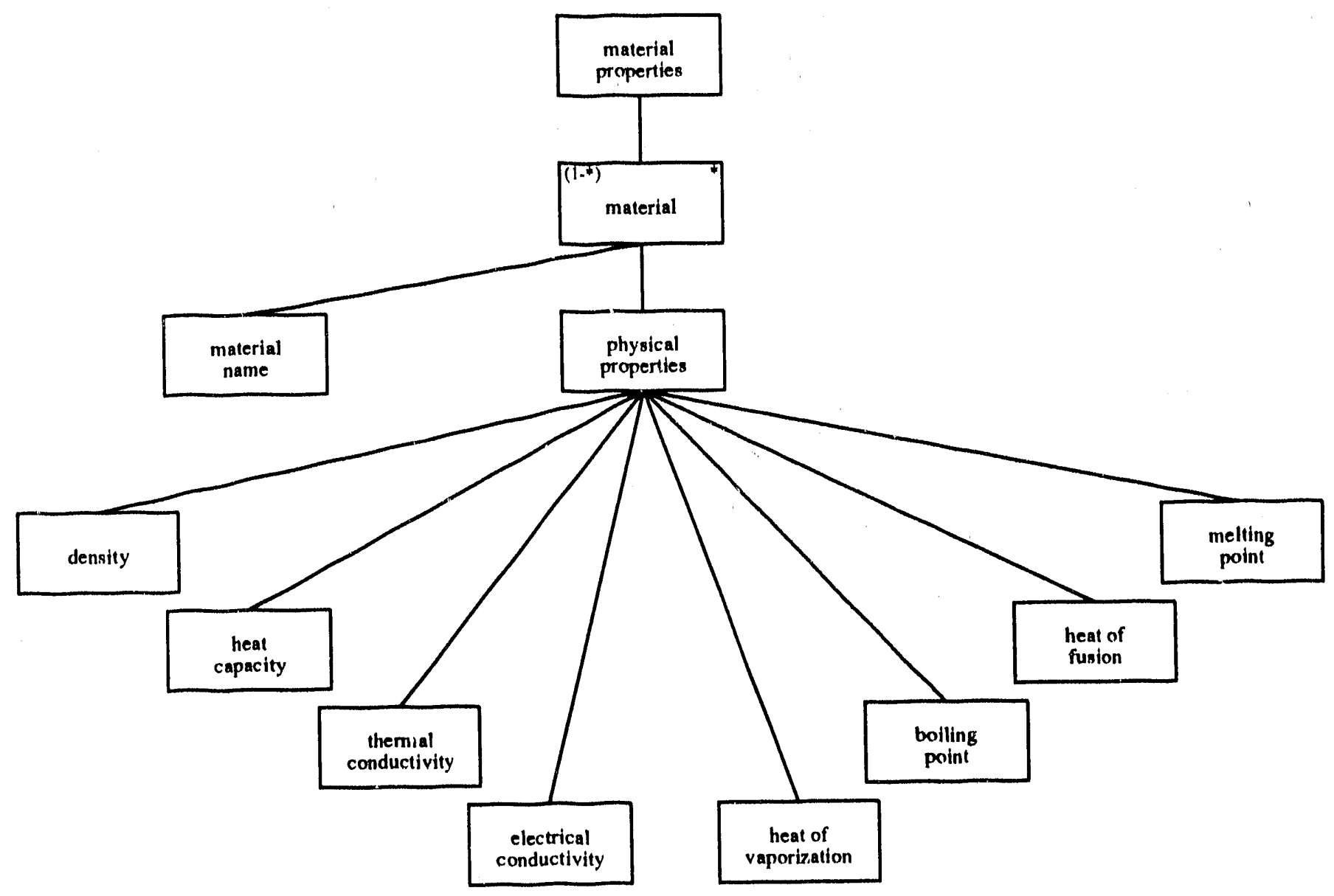


Project: In Situ Vitrification

Diagram: matrix_equation
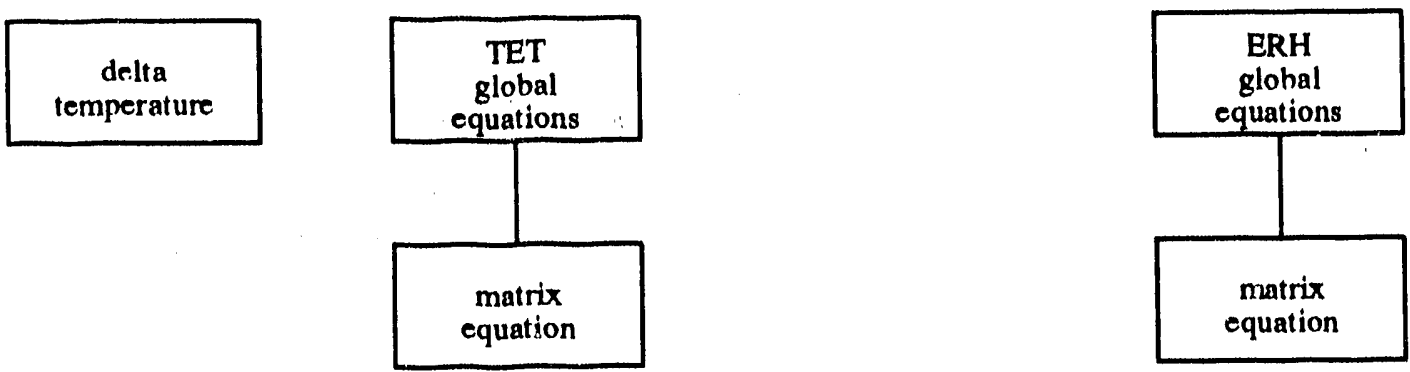

delta

potential

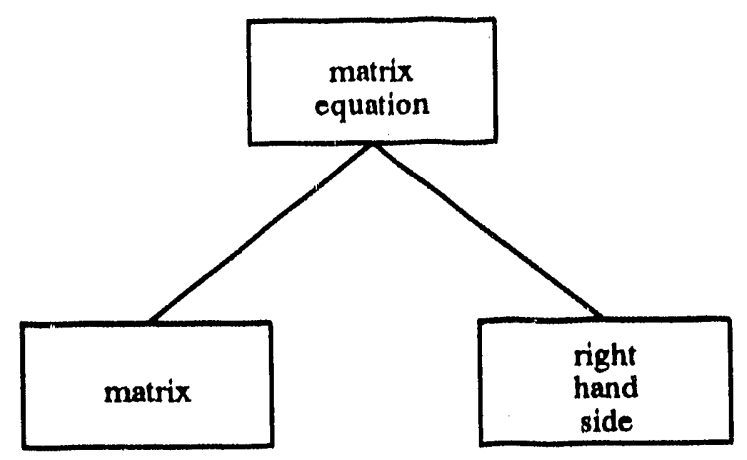

C -17 
Project: In Situ Vitrification

Diagram: misc

nodal electrode potential

boundary value

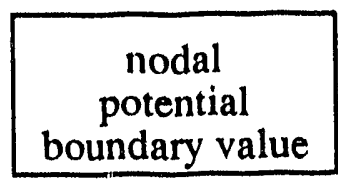

nodal

temperature

boundary value

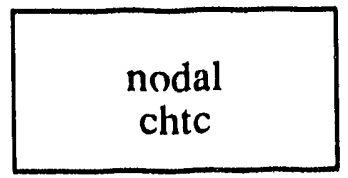

nodal

rhtc

C. 18 
Project: In Situ Vitrification

Diagram: node_variables

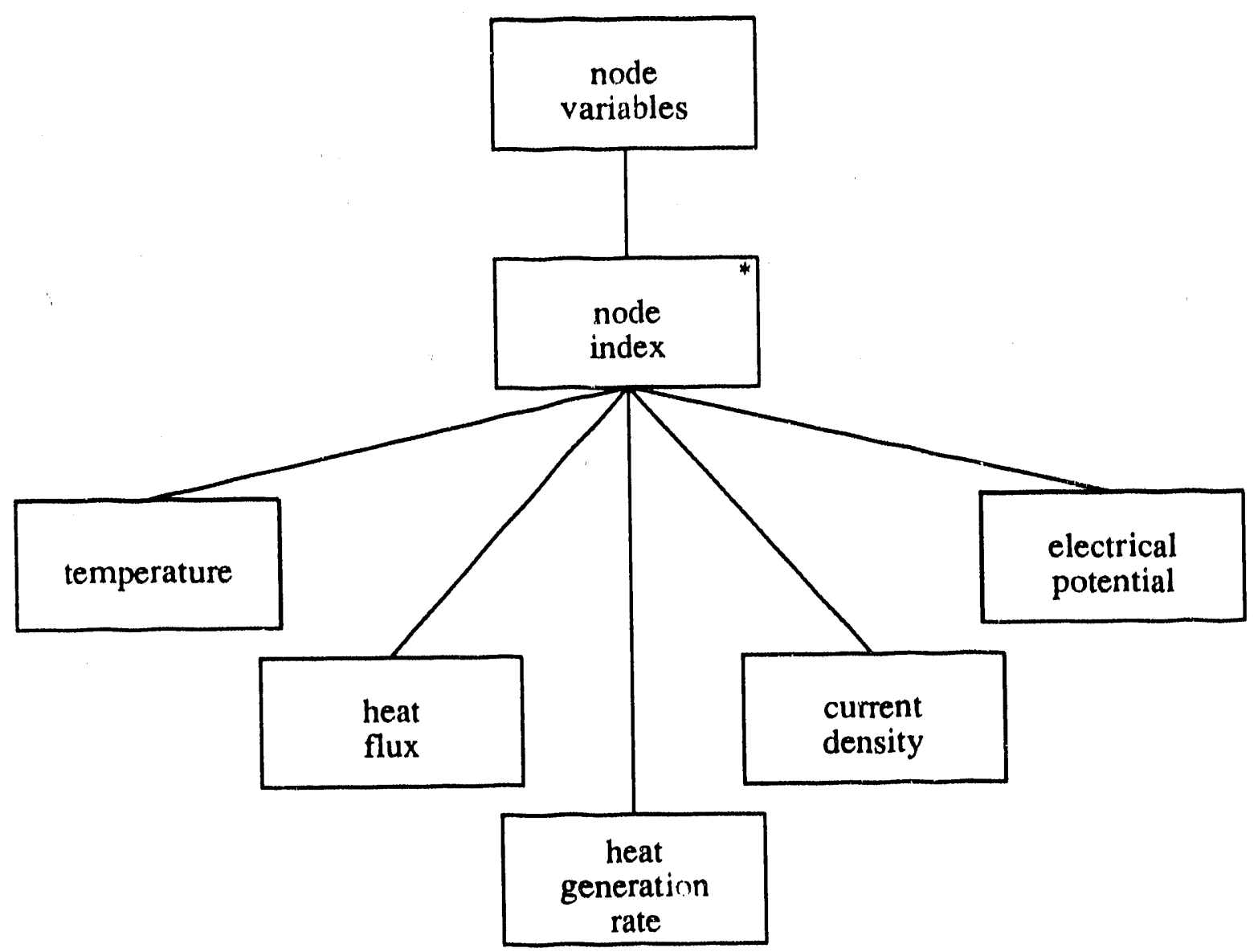


Project: In Situ Vitrification

Diagram: output_executive_io
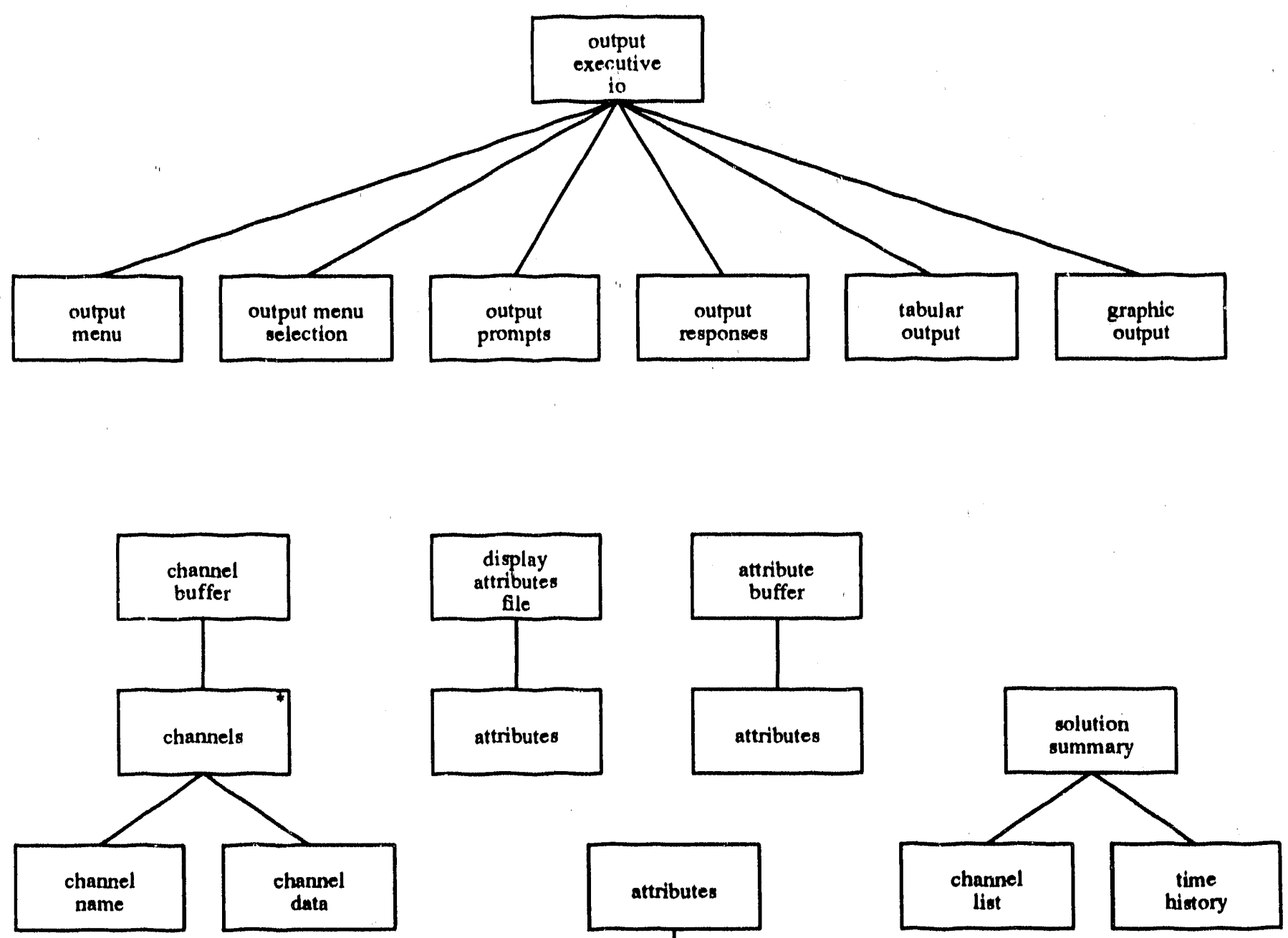

selected problem name

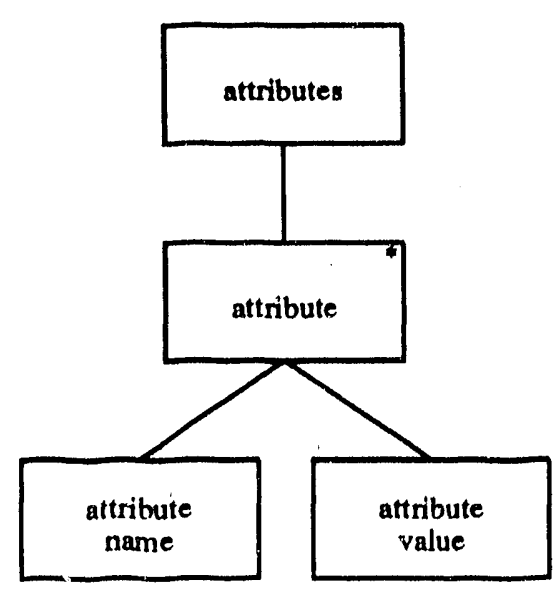


Project: In Situ Vitrification

Diagram: problem_controls

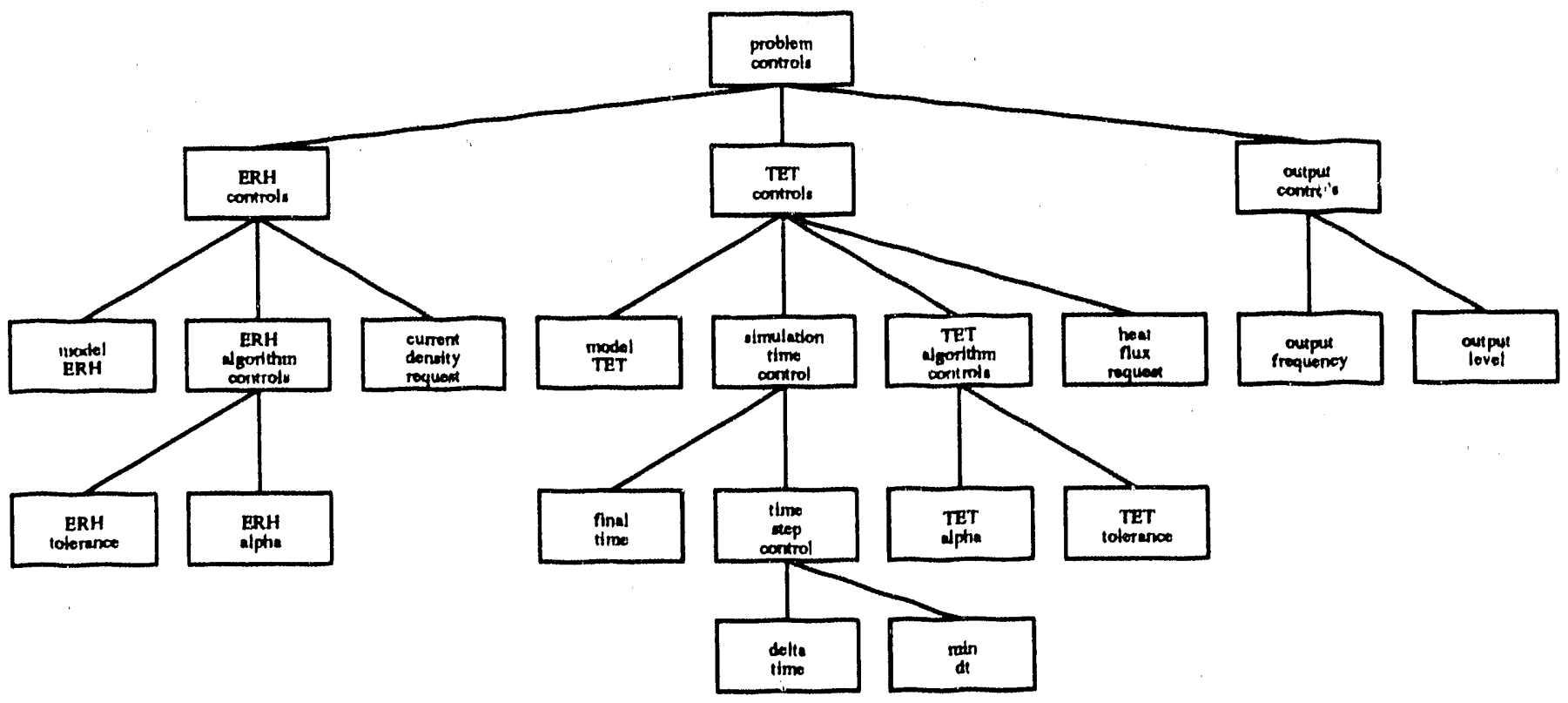


Project: In Situ Vitrification

Diagram: problem_data
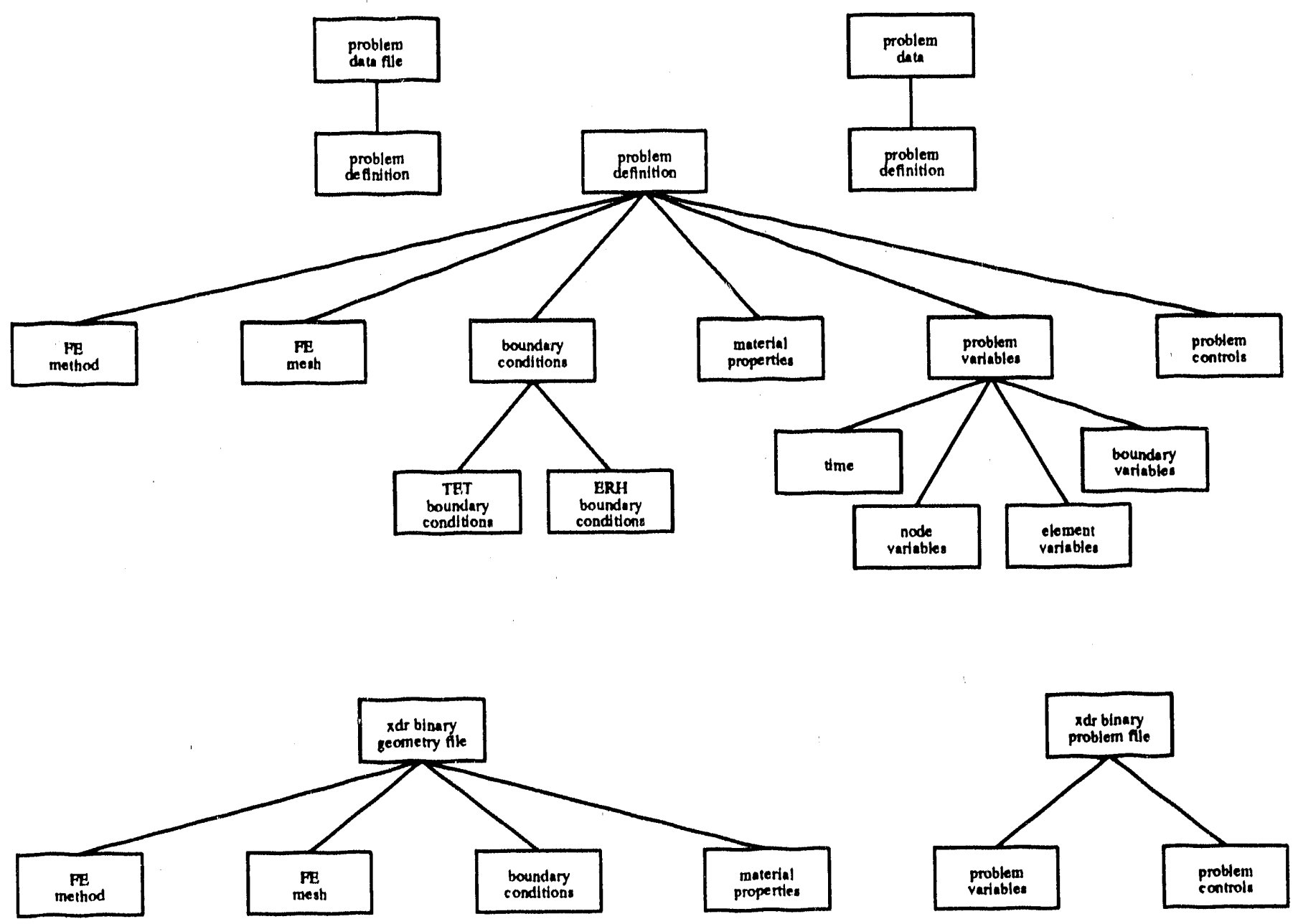
Project: In Situ Vitrification

Diagram: simulation_executive_io
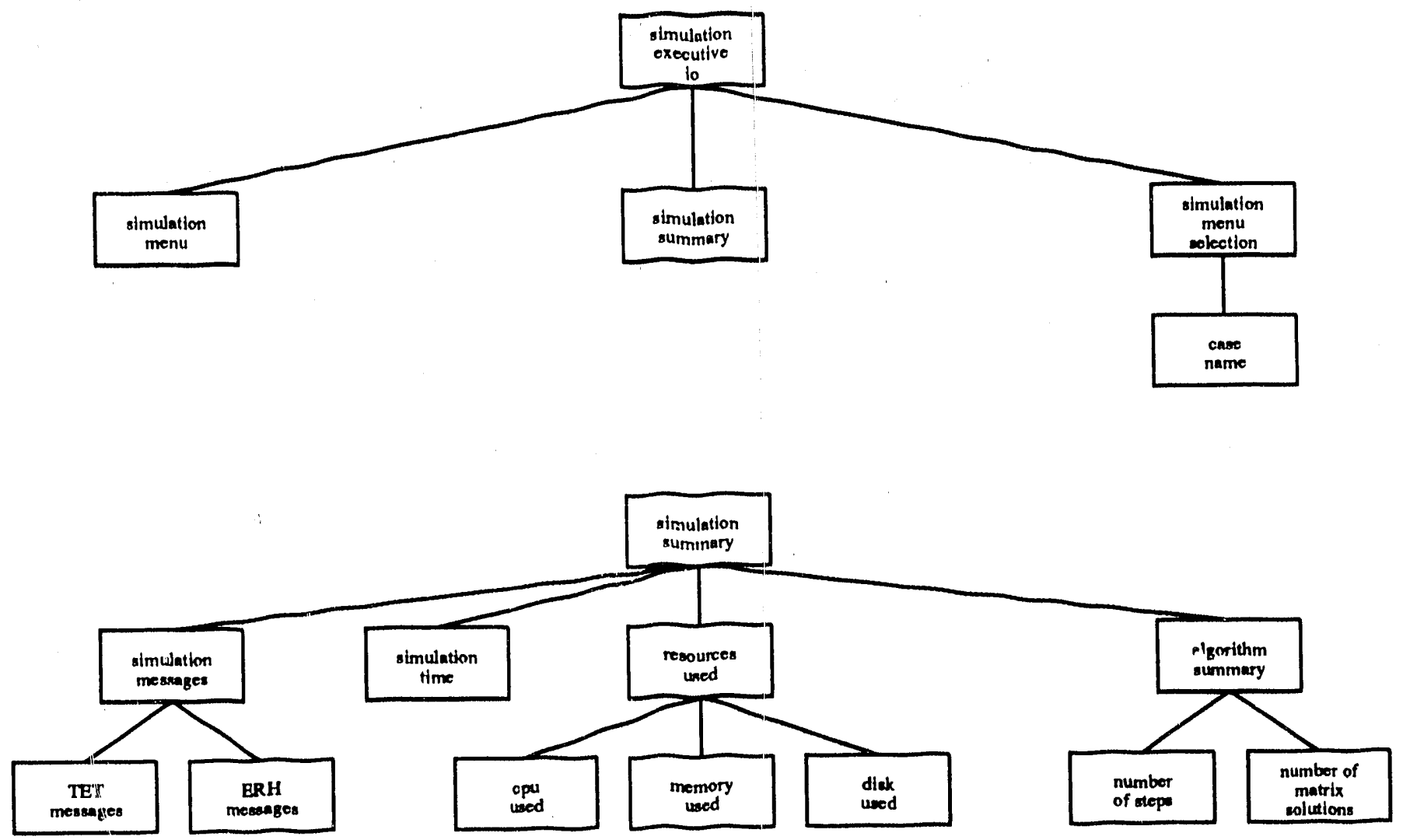
APPENDIX D - DATA DICTIONARY

D. 1 
2D_Gauss_point

Defined as Data Element in FE_method

Used in 1 Data Structure

2D_Gauss_points (Iteration)

Note DataDefinition

Type: 2D_vector

2D_derivative_values

Defined as Data Element in FE_method

Used in 1 Data Structure

2D_Gauss_point_index (Sequence)

Note DataDefinition

Type: 2D_vector

2D_function_value

Defined as Data Element in FE_method

Used in I Dala Structure

2D_Gauss_point_index (Sequence)

Note DataDefinition

Type: real

3D_Gauss_point

Defined as Data Elernent in FE_method

Used in I Data Structure

3D_Gauss_points (Iteration)

Note DataDefinition

Type: 3D_vector

3D_derivative_values

Defined as Data Element in FE_method

Used in I Data Structure

3D_Gauss_point_index (Sequence)

Note DataDefinition

Type: 3D_vector

3D_function_value

Defined as Data Element in FE_method

Used in 1 Data Structure

3D_Gauss_point_index (Sequence)

Note DataDefinition

Type: real

ERH_Dirichlet_boundary_index

Defined as Data Element in ERH_boundary_conditions

Used in I Data Structure

ERH_Dirichlet_boundary_conditions (Sequence)

Used in 2 Data Flows

DFD diagram level 3.3.4

From: ERH_boundary_conditions (Data Store)

To: electrode_position_boundary_executive (Process)

DFD diagram level 3.3.5

Fron: ERH_boundary_conditions (Data Store)

To: potential_boundary_executive (Process)

Note DalaDefinition

Type: integer

ERH_Neumann_boundary index

Definel as Datn Elemen! in ERH_hon!ndary_conditions

D - 2 
Used in I Data Structure

ERH_Neumann_boundary_condition (Sequence)

Used in 1 Data Flow

DFD diagram level 3.3.3

From: ERH_boundary_conditions (Data Store)

To: flux_boundary_executive (Process)

Note DataDefinition

\section{ERH_alpha}

Type: integer

Defined as Data $\mathrm{F}^{\text {' }}$ 'ment in problem_controls

Used in 1 Data Str:icture

ERH_algorithm_controls (Sequence)

Used in 2 Data Flows

DFD diagram level 3.3.2

From: ERH_element_equations_executive (Process)

To: accumulate_ERH_element_equation_GP_component (Process)

DFD diagram level 3.3.3

From: flux_boundary_executive (Process)

To: accumulate_flux_boundary_integral_GP_component (Process)

Note DataDefinition

Type: real

\section{ERH_messages}

Defined as Data Element in simulation_executive_io

Used in 1 Data Structure simulation_messages (Sequence)

Used in 2 Data Flows

DFD diagram level 3

From: solve_ERH_problem (Process)

To: simulation_executive (Process)

DFD diagram level 3.3

From: ERH_executive (Process)

To: Offpage.3.3 (Offpage Connector)

Note DataDefinition

Type: text

\section{ERH_tolerance}

Defined as Data Element in problem_controls

Used in I Data Structure

ERH_algorithm_controls (Sequence)

Note DataDefinition

Type: real

GP_ERH_heat_generation_rate

Defined as Data Element in GP_values

Used in I Data Flow

DFD diagram level 3.4.2

From: compute_TET_element_GP_ERH_heat_generation_rate (Process)

To: compute_TET_element_GP_heat_generation_rate (Process)

Note DataDefinition

GP_chtc

Type: real

Defined as Data Element in GP_values

Used in I Data Flow

DFD diagram level 3.4.3

From: compute_GP_chtc (Process)

To: accumulate_convection_boundary_integral_GP_component (Process)

Note Datanefinition

D - 3 
Type: real

GP_current_density

Defined as Data Element in GP_values

Used in 3 Data Flows

DFD diagram level 3.3.3

From: compute_ERH_surface_element_GP_current_density (Process)

To: accumulate_flux_boundary_integral_GP_component (Process)

DFD diagram level 3.3.7

From: compute_ERH_element_GP_current_density (Process)

To: ERH_element_current_density_executive (Process)

DFD diagram level 3.3.7

From: compute_ERH_element_GP_current_density (Process)

To: compute_ERH_element_GP_heat_generation_rate (Process)

Note DataDefinition

Type: 3D vector

GP_density_heat_capacity

Defined as Data Element in GP_values

Used in 2 Data Flows

DFD diagram level 3.4.2.5

From: compute_TET_element_GP_density_heat_capacity (Process)

To: Offpage.3.4.2.5 (Offpage Connector)

DFD diagram level 3.4.2

From: compute_TET_element_GP_thermal_quantities (Process)

Note DataDefinition

To: accumulate_TET_element_equation_GP_component (Process)

Type: real

GP_electrical_conductivity

Defined as Data Element in GP_values

Used in 4 Data Flows

DFD diagram level 3.4.2.5

From: compute_TET_element_GP_electrical_conducivity (Process)

To: Offpage.3.4.2.5 (Offpage Connector)

DFD diagram level 3.4 .2

From: compute_TET_element_GP_thermal_quantities (Process)

To: compute_TET_element_GP_heat_generation_rate (Process)

DFD diagram level 3.3.2

From: compute_ERH_element_GP_electrical_conductivity (Process)

To: accumulate_ERH_element_equation_GP_component (Process)

DFD diagram level 3.3.7

From: recompute_ERH_element_GP_electrical_conductivity (Process)

To: compute_ERH_element_GP_current_density (Process)

Note DataDefinition

Type: $3 \mathrm{D}$ vector

GP_heat_capacity

Defined as Data Element in GP_values

Used in I Data Flow

DFD diagram level 3.4.2.5

From: compute_TET_element_GP_density_heat_capacity (Process)

To: compute_TET_element_GP_phase_change_energy (Process)

Note DataDefinition

Type: real

GP_heat_flux

Defined as Data Element in GP_values

Used in I Data Flow

DFD diagram level 3.4.7 
From: compute_TET_element_GP_heat_flux (Process)

Note DataDefinition

To: TET_element_heat_flux_executive (Process)

Type: $3 \mathrm{D}$ vector

GP_heat_generation_rate

Defined as Data Ëlement in GP_values

Used in 2 Data Flows

DFD diagram level 3.4.2

From: compute_TET_element_GP_heat_generation_rnte (Process)

To: accumulate_TET_element_equation_GP_component (Process)

DFD diagram level 3.3.7

From: compute_ERH_element_GP_heat_generation_rate (Process)

To: ERH_element_current_density_executive (Process)

Note DataDefinition

Type: real

GP_phase_change_energy

Defined as Data Element in GP_values

Used in 2 Data Flows

DFD diagram level 3.4.2.5

From: compute_TET_element_GP_phase_change_energy (Process)

To: Offpage 3.4.2.5 (Offpage Connector)

DFD diagram level 3.4.2

From: compute_TET_element_GP_thermal_quantities (Process)

To: compute_TET_element_GP_heat_generation_rate (Process)

Note DataDefinition

Type: real

GP_potential

Defined as Data Element in GP_values

Used in 2 Data Flows

DFD diagram level 3.3.2

From: compute_ERH_element_GP_potential (Process)

To: compute_ERH_element_GP_electrical_conductivity (Process)

DFD diagram level 3.3.7

From: recompute_ERH_element_GP__potential (Process)

To: recompute_ERH_element_GP_electrical_conductivity (Process)

Note DataDefinition

Type: real

GP_potential_gradient

Defined as Data Element in GP_values

Used in 2 Data Flows

DFD diagram level 3.3.7

From: compute_ERH_element_GP_potential_gradient (Process)

To: compure_ERH_element_GP_current_density (Process)

DFD diagram level 3.3.7

From: compute_ERH_element_GP_potential_gradient (Process)

To: compute_ERH_element_GP_heat_generation_rate (Process)

Note DataDefinition

Type: real

GP_rhtc

Defined as Data Element in GP_values

Used in I Data Flow

DFD diagram level 3.4.4

From: compute_GP_rhtc (Process)

To: accumulate_radiation_boundary_integral_GP_component (Process)

Note DataDefinition

D - 5 
Type: real

GP_temperature

Defined as Data Element in GP_values

Used in 8 Data Flows

DFD dingram level 3.4.2.5

From: Offpage.3.4.2.5 (Offpage Connector)

To: compute_TET_element_GP_thermal_conductivity (Process)

DFD diagram level 3.4.2.5.

From: Offpage 3.4.2.5 (Offpage Connector)

To: compute_TET_element_GP_density_heat_capacity (Process)

DFD diagram level 3.4.7

From: recompute_TET_element_GP_temperature (Process)

To: recompute_TET_element_GP_thermal_conductivity (Process)

DFD diagram level 3.4.2.5

From: Offpage.3.4.2.5 (Offpage Connector)

To: compute_TET_element_GP_electrical_conducivity (Process)

DFD diagram level 3.4.2

From: compute_TET_element_GP_temperature (Process)

To: compute_TET_element_GP_thermal_quantities (Process)

DFD diagram level 3.4.3

From: compute_convection_element_GP_temperature (Process)

To: accumulate_convection_boundary_integral_GP_component (Process)

DFD diagram level 3.4.4

From: compute_radiation_element_GP_temperature (Process)

To: accumulate_radiation_boundary_integral_GP_component (Process)

DFD diagram level 3.4.2.5

From: Offpage.3.4.2.5 (Offipage Connector)

To: compute_TET_element_GP_phase_change_energy (Process)

Note DataDefinition

Type: real

GP_temperature_gradient

Defined as Data Element in GP_values

Used in I Data Flow

DFD diagram level 3.4.7

From: compute_TET_element_GP_temperature_gradient (Process)

To: compute_TET_element_GP_heat_flux (Process)

Note DataDefinition

Type: real

GP_thermal_conductivity

Defined as Data Element in GP_values

Used in 3 Data Flows

DFD diagram level 3.4.2.5

From: compute_TET_element_GP_thermal_conductivity (Process)

To: Offpage.3.4.2.5 (Offiage Connector)

DFD diagram level 3.4 .7

From: recompute_TET_element_GP_thermal_conductivity (Process)

To: compute_TET_element_GP_heat_flux (Process)

DFD diagran level 3.4.2

From: compute_TET_element_GP_thermal_quantities (Process)

To: accumulate_TET_element_equation_GP_component (Process)

Note DataDefinition

Type: $3 \mathrm{D}$ vector

ISV menu

Iefined as Data Element in ISV_executive_io

Used in I Data Structure 
ISV_executive_io (Sequence)

Used in 2 Data Flows

DFD diagram level 0

From: Executive (Process)

To: Offpage.0 (Offpage Connector)

DFD diagram level top

From: ISV (Process)

To: ISV_analyst (External)

Note DataDefinition

Type: menu

TET_Dirichlet_boundary_index

Defined as Data Elenient in TET_boundary_conditions

Used in 1 Data Structure

TET_Dirichlet_boundary_condition (Sequence)

Used in 1 Data Flow

DFD diagram level 3.4 .5

From: TET_boundary_conditions (Data Store)

To: temperature_boundary_executive (Process)

Note DataDefinition

Type: integer

TET_Neumann_boundary_index

$\bar{D}$ Defined as Data Element in TET_boundary_conditions

Used in 1 Data Structure

TET_Neumann_boundary_conditions (Sequence)

Used in 2 Data Flows

DFD diagram level 3.4.3

From: TET_.boundary_conditions (Data Store)

To: convection_boundary_executive (Process)

DFD diagram level 3.4.4

From: TET_boundary_conditions (Data Store)

To: radiation_boundary_executive (Process)

Note DataDefinition

Type: integer

\section{TET alpha}

Defined as Data Element in problem_controls

Used in 1 Data Structure

TET_algorithm_controls (Sequence)

Used in 3 Data Flows

DFD diagram level 3.4.2

From: TET_element_equations_executive (Process)

To: accumulate_TET_element_equation_GP_component (Process)

DFD diagram level 3.4.3

From: convection_boundary_executive (Process)

To: accumulate_convection_boundary_integral_GP_component (Process)

DFD diagram level 3.4.4

From: radiation_boundary_executive (Process)

To: accumulate_radiation_boundary_integral_GP_component (Process)

Note DataDefinition

Type: real

TET_messages

Defined as Data Element in simulation_executive_io

Used in I Data Siructure

simulation_messages (Sequence)

Used in 2 Data Flows

DFD diagram level 3 
From: solve_TET_prohlem (Frncess)

To: simulation_executive (Process)

DFD diagram level 3.4

From: TET_executive (Process)

To: Offpage.3.4 (Offpage Connector)

Note DataDefinition

Type: text

\section{TET tolerance}

Defined as Data Element in prob!em_controls

Used in 1 Data Structure

TET_algorithm_controls (Sequence)

Note DataDefinition

Type: real

abscissa

Defined as Data Element in correlation

Used in 1 Data Structure linear_plece (Sequence)

Note DataDefinition

Type: real

ascii_only

Defined as Data Element in input_executive_io

Used in 2 Data Flows

DFD diagram level 2

From: ISV_input_executive (Process)

To: store_new_case (Process)

DFD diagram level 2.1

From: input_dialog_manager (Process)

To: Offpage.2.1 (Offpage Connector)

Note DataDefinition

Type: logical

attribute name

Defined as Data Element in output_executive_io

Used in I Data Structure

attribute (Sequence)

Note DataDefinition

Type: string

attribute_value

Defined as Data Element in output_executive_io

Used in I Data Structure

attribute (Sequence)

Note DataDefinition

Type: alphanumeric

base case name

Defined as Data Element in input_executive_io

Used in 1 Data Structure

input_responses (Selection)

Used in 2 Data Flows

DFD diagram level 2

Fron: ISV_input_executive (Process)

To: load_base_case (Process)

DFD diagran level 2.1

From: input_dialog manager (Process)

Io: Offpage.2.1 (Ofipage Connector)

Note DataDefinition

Type: menu pick 


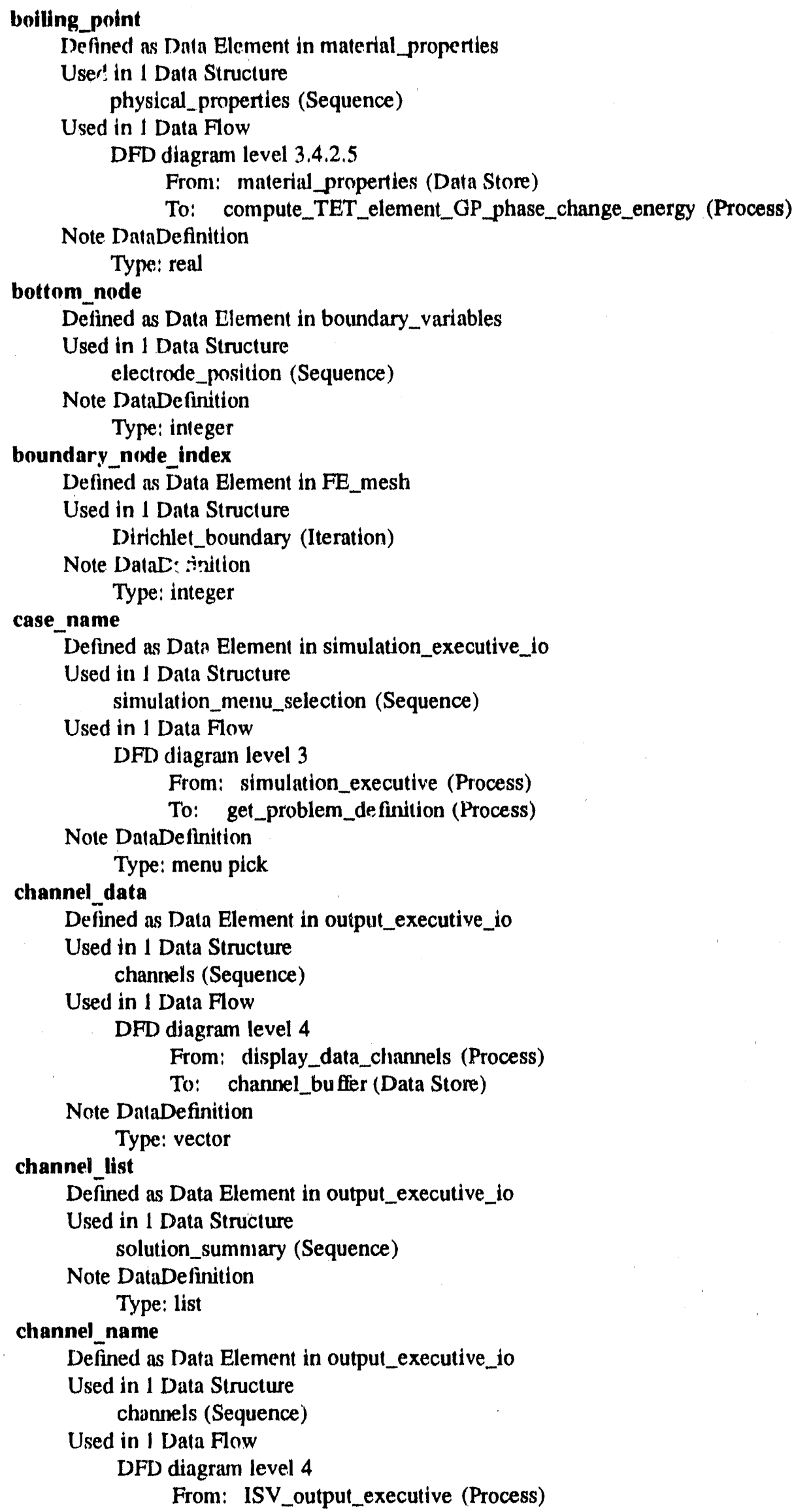


Note DataDefinition

To: channel_buffer (Data Store)

Type: siring

component_displays

Defined as Data Element in input_executive_to

Used in 1 Data Struclure

input_executive_io (Sequence)

Used in 3 Data Flows

DFD diagram level top

From: ISV (Process)

To: $1 S V_{\_}$analyst (External)

DFD diagram level 0

From: input_processor (Process)

To: Offpage.0 (Offpage Connector)

DFD diagram level 2

From: view_component (Process)

To: Offpnge.2 (Offpage Connector)

Note DataDefinition

Type: text

component_name

Defined as Data Element in input_executive_io

Used in 1 Data Structure

input_responses (Selection)

Used in 3 Data Flows

DFD diagram level 2

From: ISV_Input_executive (Process)

To: view_component (Process)

DFD diagram level 2

From: ISV_input_executive (Process)

To: modify_component (Process)

DFD diagram level 2,1

From: input_dialog_manager (Process)

To: Offpage.2.1 (Offpage Connector)

Note DataDefinition

Type: menu pick

component_new_data

Defined as Data Element in input_executive_io

Used in 1 Data Structure input_responses (Selection)

Used in 2 Data Flows

DFU diagram level 2

From: ISV_input_executive (Process)

To: modify_component (Process)

DFD diagram level 2.1

From: input_dialog manager (Process)

To: Offpage.2.1 (Offpage Connector)

Note DataDefinition

Type: numeric

consistency_flags

Defined as Data Element in input_executive_io

Used in 1 Data Structure consistency (Hteration)

Note DataDefinition

Type: logical

constant_value

D - 10 
Defined as Data Element in correlation

Used in I Data Structure correlation (Selection)

Note DataDefinition

Type: real

convection_air_temperature

Defined as Data Element in TET_boundary_conditions

Used in 1 Data Structure convection_boundary_element (Sequence)

Used in 1 Data Flow

DFD diagram level 3.4 .3

From: TET_boundary_conditions (Data Store)

To: accumulate_convection_boundary_integral_GP_component (Process)

Note DataDefinition

Type: correlation

convection_energy

Defined as Data Element in element_variables

Used in 1 Data Structure

element_energy_components (Sequence)

Used in 3 Data Flows

DFD diagram level 3.4

From: apply_TET_convection_boundary_condition (Process)

To: problem_variables (Data Store)

DFD diagram level 3

From: solve_TET_problem (Process)

To: problem_variables (Data Store)

DFD diagram level 3.4.3

From: accumulate_convection_boundary_integral_GP_component (Process)

To: problem_variables (Data Store)

Note DataDefinition

Type: real

convection heat transfer

Defined as Data Element in TET_boundary_conditions

Used in 1 Data Structure convection_boundary_element (Sequence)

Note DataDefinition

Type: correlation

cpu_estimate

Defined as Data Element in input_executive_io

Used in 1 Data Structure resources_estimate (Sequence)

Note Databefinition

Type: real

cpu_used

Defined as Datn Element in simulation_executive_io

Used in I Data Structure resources_used (Sequence)

Note DataDefinition

Type: real

current_density

Detined as Data Element in node_variables

Used in I Data Structure node_index (Sequence)

Used in 3 Data Flows

DFD diagram level 3 
Frnm: snlve_ERH

To: problem_variables (Data Store)

DFD diagram level 3.3

From: compute_ERH_element_current_density (Process)

To: problem_variables (Data Store)

DFD diagram level 3.3.7

From: ERH_element_current_density_executive (Process)

Note DataDefinition

To: problem_variables (Data Store)

Type: 3D_vector

current_density_boundary_value

Defined as Data Element in ERH_boundary_conditions

Used in 1 Data Structure current_density_boundary_element (Sequence)

Note DataDefinition

Type: correlation

current_density_request

Defined as Data Element in problem_controls

Used in I Dala Structure

ERH_controls (Sequence)

Used in 2 Data Flows

DFD diagram level 3.3

From: ERH_executive (Process)

To: compute_ERH_element_current_density (Process)

DFD diagram level 3.3 .7

From: Offpage.3.3.7 (Offpage Connector)

To: ERH_element_current_density_executive (Process)

Note DataDefinition

Type: logical

delta potential

Defined as Data Element in matrix_equation

Used in 1 Data Flow

DFD diagram level 3.3

From: solve_ERH_glnbal_equations (Process)

Note DataDefinition

To: ERH_executive (Process)

Type: vector

delta_temperature

Defined as Data Element in matrix_equation

Used in 1 Data Flow

DFL diagram level 3.4

From: solve_TET_global_equations (Process)

To: TET_executive (Process)

Note DataDefinition

Type: vector

delta time

Defined as Dila Element in problem_controls

Used in I Data Struclure time_step_control (Sequence)

Used in 2 Data Flows

DFD diagran level 3.4 .2

From: TET_element_equations_executive (Process)

To: compute_TET_element_GP_heat_generation_rate (Process)

DFD diagram level 3.4.2

From: TET_element_equations_executive (Process)

D -12 
To: accumulate_TET_element_equation_CP_component (Process)

Note DataDefinition

Type: real

density

Defined as Data Element in material_properties

Used in I Data Structure

physical_properties (Sequence)

Used in 2 Data Flows

DFD diagram level 3.4.2.5

From: material_properties (Dnta Store)

To: compute_TET_element_GP_density_heat_capacity (Process)

DFD diagram level 3.4.2.5

From: material_properties (Data Store)

To: compute_TET_element_GP_phase_change_energy (Process)

Note DataDefinition

det

Type: correlation

Defined as Data Element in 3D_physical_element

Used in 1 Data Structure

3D_physical_element (Sequence)

Note DataDefintion

Type: real

dfdx

Defined as Data Element in 3D_physical_element

Used in 1 Data Structure

derv (Sequence)

Note DataDelinition

Type: real

dfdy

Defined as Data Element in 3D_physical_element

Used in 1 Data Structure derv (Sequence)

Note DataDefinition

Type: real

dfdz

Defined as Dain Element in 3D_physical_element

Used in 1 Data Structure

derv (Sequence)

Note DataDefinition

Type: real

dis

Defined as Data Element in 2D_physical_element

Used in 1 Data Structure

2D_physical_element (Sequence)

Note DataDefinition

Type: real

disk estimate

Defined as Data Element in input_executive_io

Used in I Data Structure

resources_estimate (Sequence)

Note DataDefinition

Type: integer

disk used

Defined as Data Element in simulation_executive_io

Used in 1 Data Structure 


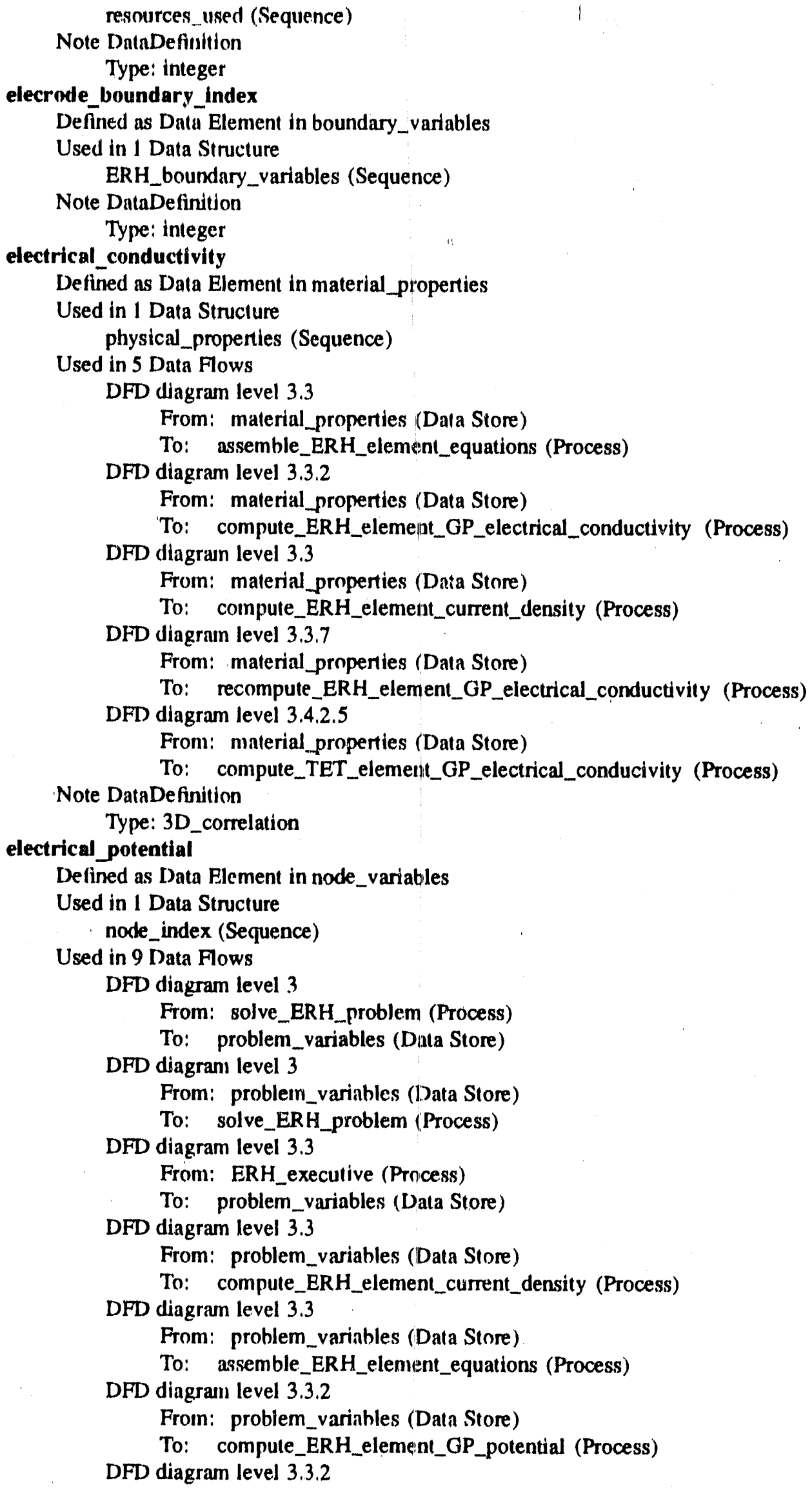


From: problem_variahles (Data Store)

To: accumulate_ERH_element_equation_GP_component (Process)

DFD diagram level 3.3 .7

From: problem_variables (Data Store)

To: recompute_ERH_element_GP_potential (Process)

DFD diagram level 3.3.7

From: problem_variables (Data Store)

To: compute_ERH_element_GP_potential_gradient (Process)

Note DataDefinition

Type: real

electrode_potential_value

Defined as Data Element in ERH_boundary_conditions

Used in 1 Data Structure

electrode_boundary_node (Sequence)

Note DataDefunition

Type: correlation

element_node_index

Defined as Data Element in FE_mesh

Used in 1 Data Structure

element (Iteration)

Note DataDefinition

Type: integer

fen

Defined as Data Element in 3D_physical_element

Used in 1 Data Structure

function_values (Iteration)

Used in 5 Data Flows

DFD diagram level 3.3.2

From: 3D_ERH_element (Dala Store)

To: compute_ERH_element_GP_potential (Process)

DFD diagram level 3.3.7

From: 3D_ERH_flux_element (Data Store)

To: recompute_ERH_element_GP_potential (Process)

DFD diagram level 3.4.2

From: 3D_TET_element (Data Store)

To: compute_TET_element_GP_temperature (Process)

DFD diagram level 3.4.7

From: 3D_TET_flix_element (Data Store)

To: recompute_TET_element_GP_temperature (Process)

DFD diagram level 3.4 .2

From: 3D_7ET_element (Data Store)

To: compute_TET_element_GP_ERH_heat_generation_rate (Process)

Note DataDefinition

Type: real

final time

Defined as Data Element in problem_controls

Used in 1 Data Struclure simulation_time_control (Sequence)

Note DataDefinition

Type: real

func

Defined as Data Element in 2D_physical_element

Used in 1 Data Structure

2D_function_values (Iteration)

Used in 5 Data Flows 
DFD diagram level 3.3.3

From: 2D_ERH_flux_element (Data Store)

To: compute_ERH_surface_element_GP_current_density (Process)

DFD diagram level 3.4 .3

Fron: 2D_TET_convection_element (Data Store)

To: compute_GP_chtc (Process)

DFD diagram level 3.4.4

From: 2D_TET_radintion_element (Data Store)

To: compute_GP_rhtc (Process)

DFD dingram level 3.4.3

From: 2D_TET_convection_element (Data Store)

To: compute_convection_element_GP_temiperature (Process)

DFD diagram level 3.4.4

From: 2D_TET_radiation_element (Data Store)

To: compute_radiation_element_GP_temperature (Process)

Note DataDefinition

Type: real

functional_correlation

Defined as Data Element in correlation

Used in 1 Data Structure

correlation (Selection)

Note DataDefinition

Type: string

graphic_output

Defined as Data Element in output_executive_io

Used in I Data Structure

output_executive_io (Sequence)

Used in 3 Data Flows

DFD diagram level 0

From: output_processor (Process)

To: Offpage.0 (Offpage Connector)

DFD diagram level top

From: ISV (Process)

To: ISV_analyst (External)

DFD diagram level 4

From: display_data_channels (Process)

To: Offpage.4 (Offpage Connector)

Note DataDefinition

Type: graphic

heat_capacity

Defined as Data Element in material_properties

Used in 1 Data Structure

physical_properties (Sequence)

Used in I Data Flow

DFD diagram level 3.4.2.5

From: material_properties (Data Store)

To: compute_TET_element_GP_density_heat_capacity (Process)

Note DataDefinition

Type: correlation

heat_flux

Detined as Data Element in nocde_variables

Used in I Data Structure nock_index (Sequence)

Usod in 3 Data Plows

DFD diagram level 3 
From: solve TET_prohlem (Process)

To: problem_variables (Data Store)

DFD diagram level 3.4.7

From: TET_element_heat_flux_executive (Process)

To: problem_variables (Data Store)

DFD diagram level 3.4

From: compute_TET_element_heat_flux (Process)

Note DataDefinition

To: problem_variables (Data Store)

Type: 3D_vector

heat_flux_request

Defined as Data Element in problem_controls

Used in 1 Data Structure

TET_controls (Sequence)

Used in 2 Data Flows

DFD diagram level 3.4 .7

From: Offpage.3.4.7 (Offpage Connector)

To: TET_element_heat_flux_executive (Process)

DFD diagram level 3.4

From: TET_executive (Process)

To: compute_TET_element_heat_flux (Process)

Note DataDefinition

Type: logical

heat_generation_rate

Defined as Data Element in node_variables

Used in 1 Data Structure

node_index (Sequence)

Used in 6 Data Flows

DFD diagram level 3

From: solve_ERH_problem (Process)

To: problem_variables (Data Store)

DFD diagram level 3.3.7

From: ERH_element_current_density_executive (Process)

To: problem_variabies (Data Store)

DFD diagram level 3.3

From: compute_ERH_element_current_density (Process)

To: problem_variables (Data Store)

DFD diagram level 3

From: problem_variables (Data Store)

To: solve_TET_problem (Process)

DFD diagram level 3.4

From: problem_variables (Data Store)

To: assemble_TET_element_equations (Process)

DED diagram level 3.4.2

From: problem_variables (Data Store)

To: compute_TET_element_GP_ERH_beat_generation_rate (Process)

Note DataDefinition

heat_of_fusion

Type: real

Defined as Data Element in material_properties

Used in 1 Data Structure

physical_properties (Sequence)

Used in I Data Flow

DFD diagram level 3.4.2.5

From: material_properties (Data Store)

D -17 
To: compute_TET_element_GP_phase_change_energy (Process)

Note DalaDefinition

Type: real

heat_of_vaporization

Defined as Data Element in material_properties

Used in 1 Data Structure

physical_properties (Sequence)

Used in 1 Data Flow

DFD diagram level 3.4.2.5

From: material_properties (Data Store)

To: compute_TET_element_GP_phase_change_energy (Process)

Note DataDefinition

Type: real

independent_variable

Defined as Data Element in correlation

Used in 1 Data Structure

piecewise_linear_correlation (Sequence)

Note DataDefinition

Type: string

input_menu

Defined as Data Element in input_executive_io

Used in 1 Data Structure

input_executive_io (Sequence)

Used in 4 Data Flows

DFD diagram level 0

From: input_processor (Process)

To: Offpage.0 (Offpage Connector)

DFD diagram level 2

From: ISV_input_executive (Process)

To: Offpage.2 (Offuge Connector)

DFD diagram level top

From: ISV (Process)

To: ISV_analyst (Extemal)

DFD diagram level 2.1

From: input_dialog_manager (Process)

To: Ofpage.2.: (Offpage Connector)

Note DataDefinition

Type: menu

input_prompts

Defined as Data Element in imput_executive_io

Used in 1 Data Structure

input_executive_io (Sequence)

Used in 4 Data Flows

DFD diagram level 0

From: input_processor (Process)

To: Offpage.0 (Offpage Connector)

DFD diagram level top

From: ISV (Process)

To: ISV_analyst (External)

DFD diagram level 2

From: ISV_input_executive (Process)

To: Offpage.2 (Offpage Connector)

DFD diagram level 2.1

From: imput_dialog_manager (Process)

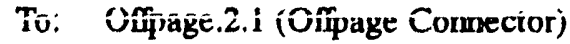


Note DataDefinition

Type: text

input_selection

Defined as Data Element in ISV_executive_io

Used in 1 Data Structure

ISV_menu_selection (Selection)

Used in 3 Data Flows

DFD diagram level 0

From: Executive (Process)

To: input_processor (Process)

DFD diagram level 2

From: Offpage. 2 (Offpage Connector)

To: ISV_input_executive (Process)

DFD diagram level 2.1

From: Offpage.2.1 (Offpage Connector)

To: input_dialog_manager (Process)

Note DataDefinition

Type: menu pick

\section{load_selection}

Defined as Data Element in input_executive_io

Used in 1 Data Structure

input_menu_selection (Selection)

Note DataDefinition

mass_density

Type: menu pick

Defined as Data Element in element_variables

Used in I Data Structure material_index (Sequence)

Note DalaDefinition

Type: real

material name

Defined as Data Element in material_properties

Used in I Data Structure material (Sequence)

Note DataDefinition

Type: string

matrix

Defined as Data Element in matrix_equation

Used in 1 Data Structure matrix_equation (Sequence)

Note DataDefinition

Type: matrix

melting point

Defined as Datn Element in material_properties

Used in I Data Structure physical_properties (Sequence)

Used in I Data Flow

DFD diagramn level 3.4.2.5

From: material_properties (Data Store)

To: compute_TET_element_GP_phase_change_energy (Process)

Note DataDefinition

Type: real

memory_estimate

Defined as Data Element in input_executive_io

Used in 1 Data Structure 


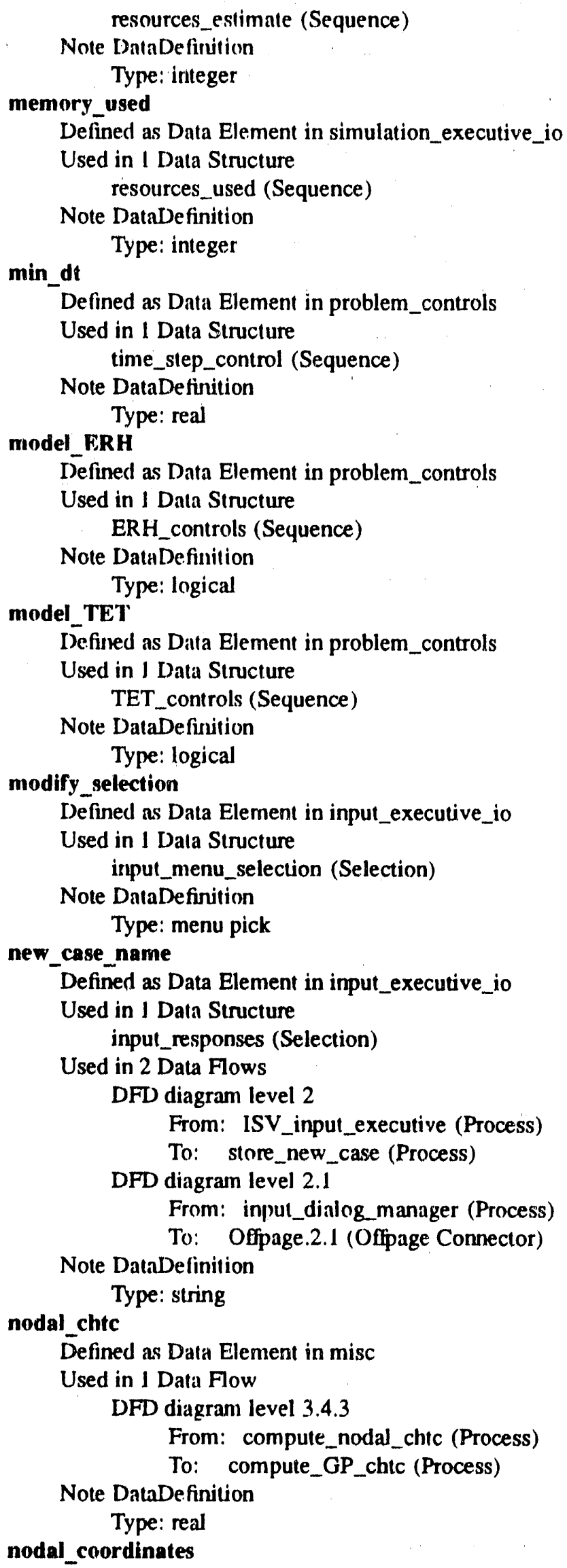


Defined as Data Element in FE_mesh

Used in 1 Data Structure

node (Iteration)

Used in 7 Data Flows

DFD diagram level 3.4.2

From: FE_mesh (Data Store)

To: compute_TET_element_shape_function_GP_values (Process)

DFD diagram level 3.4 .7

From: FE_mesh (Data Store)

To: recompute_TET_element_shape_function_GP_values (Process)

DFD diagram level 3.4 .3

From: FE_mesh (Data Store)

To: compute_convection_element_shape_function_GP_values (Process)

DFD diagram level 3.4 .4

From: FE_mesh (Data Store)

To: compute_radiation_element_shape_function_GP_values (Process)

DFD diagram level 3.3.2

From: FE_mesh (Data Store)

To: compute_ERH_elenent_shape_function_GP_values (Process)

DFD diagram level 3.3.3

From: FE_mesh (Data Store)

To: compute_flux_element_shape_function_GP_values (Process)

DFD diagram level 3.3.7

From: FE_mesh (Data Store)

To: recompute_ERH_element_shape_function_GP_values (Process)

Note DataDefinition

Type: 3D_vector

nodal_electrode_potential_boundary_value

Defined as Data Element in misc

Used in 1 Data Flow

DFD diagram level 3.3 .4

From: get_nodal_electrode_potential_boundary_value (Process)

Note DataDefinition

To: electrode_position_boundary_executive (Process)

Type: real

nodal_potential_boundary_value

Defined as $\bar{D}$ ata Element in misc

Used in 1 Data Flow

DFD diagram level 3.3 .5

From: get_nodal_potential_boundary_value (Process)

To: potential_boundary_executive (Process)

Note DataDefinition

Type: real

nodal rhtc

Defined as Data Element in misc

Used in 1 Data Flow

DFD diagram level 3.4.4

From: compute_nodal_rhtc (Process)

To: compute_GP_rhtc (Process)

Note DataDefinition

Type: real

nodal_temperature_boundary_value

Defined as Data Element in misc

Used in 1 Data Flow

DFD diagram level 3.4 .5 
Frnm: get .nndal..temperature._boundnry_value (Process)

To: lemperature_boundary_executive (Process)

Note DataDefinition

Type: real

number_of_matrix_solutions

Defined as Data Element in simulation_executive_to

Used in 1 Data Structure algorithm_summary (Sequence)

Note DataDefinition

Type: integer

number of steps

Defined as Data Element in simulation_executive_io

Used in 1 Data Structure algorithm_summary (Sequence)

Note DataDefinition

ordinate

Type: integer

Iefined as Data Element in correlation

Used in 1 Data Structure

linear_piece (Sequence)

Note DataDefinition

Type: reai

outpist_frequency

Defined as Data Element in problem_controls

Used in 1 Data Structure output_controls (Sequence)

Note DataDefinition

output_level

Type: integer

Défined as Data Element in problem_controls

Used in 1 Data Structure nutput_controls (Sequence)

Used in 1 Data Flow

DFD diagram level 3

From: simulation_executive (Process)

To: write_results (Process)

Note DataDefinition

Type: integer

\section{output_menu}

Défined as Data Element in output_executive_io

Used in 1 Data Structure

output_executive_io (Sequence)

Used in 3 Data Flows

DFD diagram level 0

Fronı: outpul_processor (Process)

To: Offpage.0 (Offpage Connector)

DFD diagram level top

From: ISV (Process)

To: ISV_analyst (Extemal)

DFD diagram level 4

From: ISV_output_executive (Prrcess)

To: Offpage. 4 (Olipage Connector)

Note DataDefinition

Type: menu

output_menu_selection 
Defined as Datn Element in output_executive_to

Used in I Data Structure

output_executive_in (Sequence)

Used in 3 Data Flows

DFD diagram level top

Fron: ISV_analyst (External)

To: ISV (Process)

DFD diagram level 0

From: Offpage. 0 (Offpage Connector)

To: output_processor (Process)

DFD diagram level 4

From: Offpage.4 (Offpage Connector)

To: ISV_output_executive (Process)

Note DataDefinition

Type: menu pick

output_prompts

Defined as Data Element in output_executive_io

Used in 1 Data Structure

output_executive_io (Sequence)

Used in 3 Data Flows

DFD diagram level 0

From: output_processor (Process)

To: Offpage. 0 (Offipage Connector)

DFD diagram level top

From: ISV (Process)

To: ISV_analyst (External)

DFD diagram level 4

From: ISV_ournut_executive (Process)

To: Offpage.4 (Offpage Connector)

Note DataDefinition

Type: lext

output responses

Defined as Datn Element in output_executive_io

Used in I Data Structure

output_executive_io (Sequence)

Used in 3 Data Flows

DFD diagram level 0

From: Offpage. 0 (Offpage Connector)

To: output_processor (Process)

DFD diagram level top

Fron: ISV_analyst (External)

To: ISV (Process)

DFD diagram level 4

From: Offpage.4 (Offpage Connector)

To: ISV_output_executive (Process)

Note Datadefinition

Type: menu pick

output_selection

Défined as Data Element in ISV_executive_io

Used in 1 Data Structure

ISV_menu_selection (Selection)

Used in 2 Data Flows

DFD diagram level 0

From: Executive (Process)

To: output_processor (Process) 
DFD diagram level 4

From: Offpage.4 (Offpage Connector)

To: ISV_output_executive (Process)

Note DataDefinition

Type: menu pick

potential_boundary_value

Defined as Data Element in ERH_boundary_.conditions

Used in 1 Data Structure

potential_boundary_node (Sequence)

Note DataDefinition

Type: correlation

radiation_air_temperature

Defined as Data Element in TET_boundary_conditions

Used in 1 Data Structure

radiation_boundary_element (Sequence)

Used in I Dnta Flow

DFD diagram level 3.4 .4

From: TET_boundary_conditions (Data Store)

To: accumulate_radiation_boundary_integral_GP_component (Process)

Note Dalabefinition

Type: correlation

radiation_energy

Defined as Data Element in element_variables

Used in 1 Data Structure

element_energy_components (Sequence)

Used in 3 Dara Flows

DFD dingram level 3.4

From: apply_TET_radiation_boundary_condition (Process)

To: problem_variables (Data Store)

DFD diagram level 3

From: solve_TET_problem (Process)

To: problem_variables (Data Store)

DFD diagram level 3.4.4

From: accumulate_radiation_boundary_integral_GP_component (Process)

To: problem_variables (Data Store)

Note DataDefinition

Type: real

radiation_heat_transfer

Defined as Data Element in TET_boundary_conditions

Used in 1 Data Structure

radiation_boundary_element (Sequence)

Note DataDefinition

Type: correlation

right_hand_side

Detined as Data Element in matrix_equation

Used in 1 Data Structure matrix_equation (Sequence)

Note DataDefinition

Type: vector

selected_problem_name

Defined as Data Element in output_executive_io

Used in I Data Flow

DFD diagram level 4

From: ISV_output_executive (Process)

To: load_general_problem_data (Process) 
Note DataDefinition

Type: string

simulate_selection

Defined as Data Element in ISV_executive_to

Used in I Data Structure

ISV_menu_selection (Selection)

Used in 2 Data Flows

DFD diagram level 0

From: Executive (Process)

To: simulate_ERH_and_TET_process (Process)

DFD diagram level 3

From: Offpage. 3 (Offpage Connector)

To: simulation_executive (Process)

Note DataDefinition

Type: menu pick

simulation_menu

Defined as Data Element in simulation_executive_io

Used in I Data Structure

simulation_executive_io (Sequence)

Used in 3 Data Flows

DFD diagram level 0

From: simulate_ERH_and_TET_process (Process)

To: Offpage. 0 (Offpage Connector)

DFD diagram level 3

From: simulation_executive (Process)

To: Offpage. 3 (Offpage Connector)

DFD diagram level Iop

From: ISV (Process)

To: ISV_analyst (External)

Note DataDefinition

Type: menu

simulation_time

Defined as Data Element in simulation_executive_io

Used in I Data Structure

simulation_summary (Sequence)

Note DataDefinition

Type: real

solid_fraction

Defined as Data Element in element_variables

Used in I Data Structure

material_index (Sequence)

Used in 4 Data Flows

DFD diagram level 3

From: problem_variables (Data Store)

To: solve_TET_problem (Process)

DFD diagram level 3.4.2

From: problem_variables (Data Store)

To: compute_TET_element_GP_thermal_quantities (Process)

DFD diagran level 3.4

From: problem_variables (Data Store)

To: assemble_TET_element_equations (Process)

DFD diagram level 3.4.2.5

From: problem_variables (Data Store)

To: compute_TET_element_GP_phase_change_energy (Process)

Note DataDefinition 
Type: real

\section{store selection}

Defined as Data Element in input_executive_to

Used in 1 Data Structure input_menu_selection (Selection)

Note DataDefinition

Type: menu pick

surface element node index

Defined as Data Element in FE_mesh

Used in 1 Data Structure surface_element (Iteration)

Note DataDefinition

tabular_output

Type: integer

Defined as Data Element in outpul_executive_io

Used in 1 Data Structure

output_executive_io (Sequence)

Used in 3 Data Flows

DFD diagram level 0

From: output_processor (Process)

Tn: Offpage.0 (Offpage Connector)

DFD diagram level lop

From: ISV (Process)

Tn: ISV_analyst (External)

DFD diagram level 4

From: display_data_channels (Process)

To: Offpage 4 (Offage Connector)

Note DataDefinition

Type: text

\section{temperature}

Defined as Data Element in node_variables

Used in I Data Structure node_index (Sequence)

Used in 20) Data Flows

DFD diagram level 3

From: solve_TET_problem (Process)

To: problem_variables (Data Store)

DFD diagram level 3

From: problem_variahles (Data Store)

To: solve_ERH_problem (Process)

DFD diagram level 3

From: problem_vartables (Data Store)

To: solve_TET_problem ("'rocess)

DFD diagram level 3.4

From: TET_executive (Process)

To: problem_variables (Data Store)

DFD diagram level 3.4

From: problem_variables (Data Store)

To: compute_TET_element_heat_flux (Process)

DFD diagram level 3.4

From: problem_variables (Daia Store)

To: assemble_TET_element_equations (Process)

DFD diagram level 3.4

From: problem_variables (Data Store)

To: apply_TET_convection_boundary_condition (Process) 
DFD diagram level 3.4

From: problem_variahles (Dnta Store)

To: apply_TET_radiation_boundary_condition (Process)

DFD diagram level 3.3

From: problem_variables (Data Store)

To: assemble_ERH_element_equations (Process)

DFD diagran level 3.3.2

From: problem_variables (Data Store)

To: compute_ERH_element_GP_electrical_conductivity (Process)

DFD diagram level 3.4 .2

From: problem..variables (Data Store)

To: accumulate_TET_element_equation_GP_component (Process)

DFD diagram level 3.4.3

From: problem_variables (Dnta Sinre)

To: compute_nodal_chtc (Process)

DFD diagram level 3.4.4

Fron: problem_variables (Data Store)

To: compute_nodal_rhtc (Pricess)

DFD diagram level 3.4 .2

From: problem_variables (Data Store)

To: compute_TET_element_GP_temperature (Process)

DFD diagram level 3.4.3

From: problem_variables (Data Store)

To: compute_convection_element_GP_temperature (Process)

DFD diagram level 3.4.4

From: problem_variables (Data Store)

To: compute_radiation_element_GP_temperature (Process)

DFD diagram level 3.4.7

From: problem_variables (Datu Store)

To: recompute_TET_element_GP_temperature (Process)

DFD diagram level 3.4.7

Fron: problem_variables (Data Store)

To: compute_TET_element_GP_temperature_gradient (Process)

DFD diagram level 3.3

From: problem_variables (Data Store)

To: compute_ERH_element_current_density (Process)

DFD diagram level 3.3.7

From: problem_variables (Data Store)

To: recompute_ERH_element_GP_electrical_conductivity (Process)

Note DataDefinition

Type: real

temperature_boundary_value

Defined as Data Element in TET_boundary_conditions

Used in 1 Dala Structure

temperature_boundary_node (Sequence)

Note DataDefinition

Type: correlation

thermal conductivity

Defined as Data Element in material_properties

Used in 1 Data Structure

physical_properties (Sequence)

Used in 3 Data Flows

DFD diagram level 3.4.7

Fron: material_properties (Data Store)

To: recompute_TET_element_GP_thermal_conductivity (Process)

D - 27 
DFD dingram level 3,4

From: material_properties (Data Store)

To: compute_TET_elernent_heat_flux (Process)

DFD diagram level 3.4.2.5

From: material_propertiles (Data Store)

To: compute_TET_elernent_GP_thermal_conductivity (Process)

Note DataDefinition

time

Type: 3D_correlation

Defined as Data Element in problem data

Used in 1 Data Structure

problem_variables (Sequence)

Used in 2 Data Flows

DFD diagram level 3

From: solve_TET_problem (Process)

To: problem_variables (Data Store)

DFD diagram level 3.4

From: TET_executive (Process)

To: problem_variables (Data Store)

Note DataDefinition

Type: real

time_history

Defined as Data Element in output_executive_io

Used in I Data Structure solution, summary (Sequence)

Note DataDefinition

Type: list

top_node

Defined as Data Element in boundary_variables

Used in I Data Struclure electrode_position (Sequence)

Note IataDefinition

view_selection

Type: integer

Defined as Data Element in input_executive_io

Used in 1 Data Structure input_menu_selection (Selection)

Note DataDefinition

Type: menu pick

$x_{-}$component

Defined as Data Element in 3D_vector

Used in 1 Data Structure

3D_vector (Sequence)

Note DataDefinition

Type: real

x_component_correlation

Defined as Data Element in 3D_correlation

Used in 1 Data Structure

3D_correlation (Sequence)

Note DataDefinition

Type: correlation

y_component

Defined as Data Element in 3D_vector

Used in 1 Data Structure

3D_vector (Sequence) 
Note DataDefinition

Type: real

y_component_correlation

Defined as Data Element in 3D_correlation

Used in 1 Data Structure

3D_correlation (Sequence)

Note DataDefinition

Type: correlation

z_component

Defined as Data Element in 3D_vector

Used in 1 Data Structure

3D_vector (Sequence)

Note DataDefinition

Type: real

z_component_correlation

Defined as Data Element in 3D_correlation

Used in 1 Data Structure

3D_correlation (Sequence)

Note DataDefinition

Type: correlation 

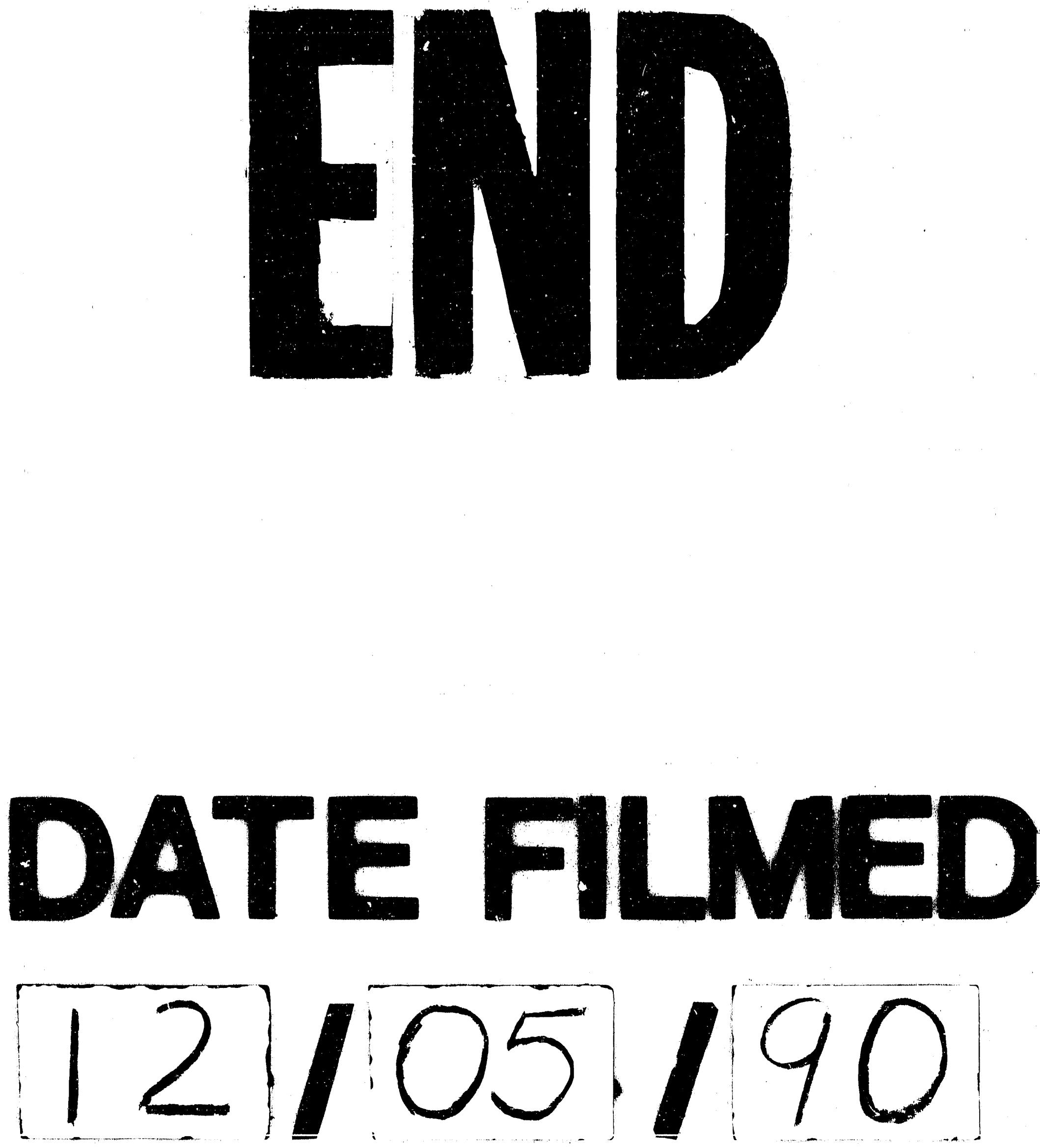
